\title{
Thermodynamics of interfaces
}

Thermodynamics of interfaces and adsorption, surface tension, contact angle, wetting. Interaction between surfaces and stabilisation mechanisms (foams, emulsions).

Antonio Stocco

Institut Charles Sadron, Strasbourg

Mcube Team

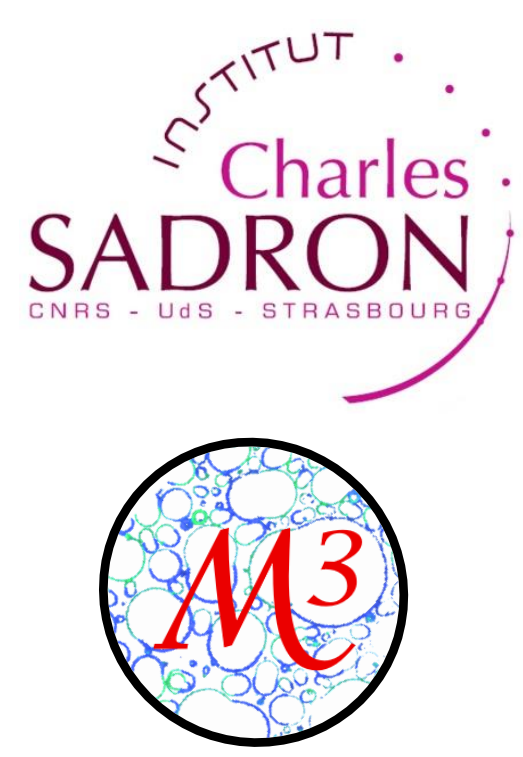




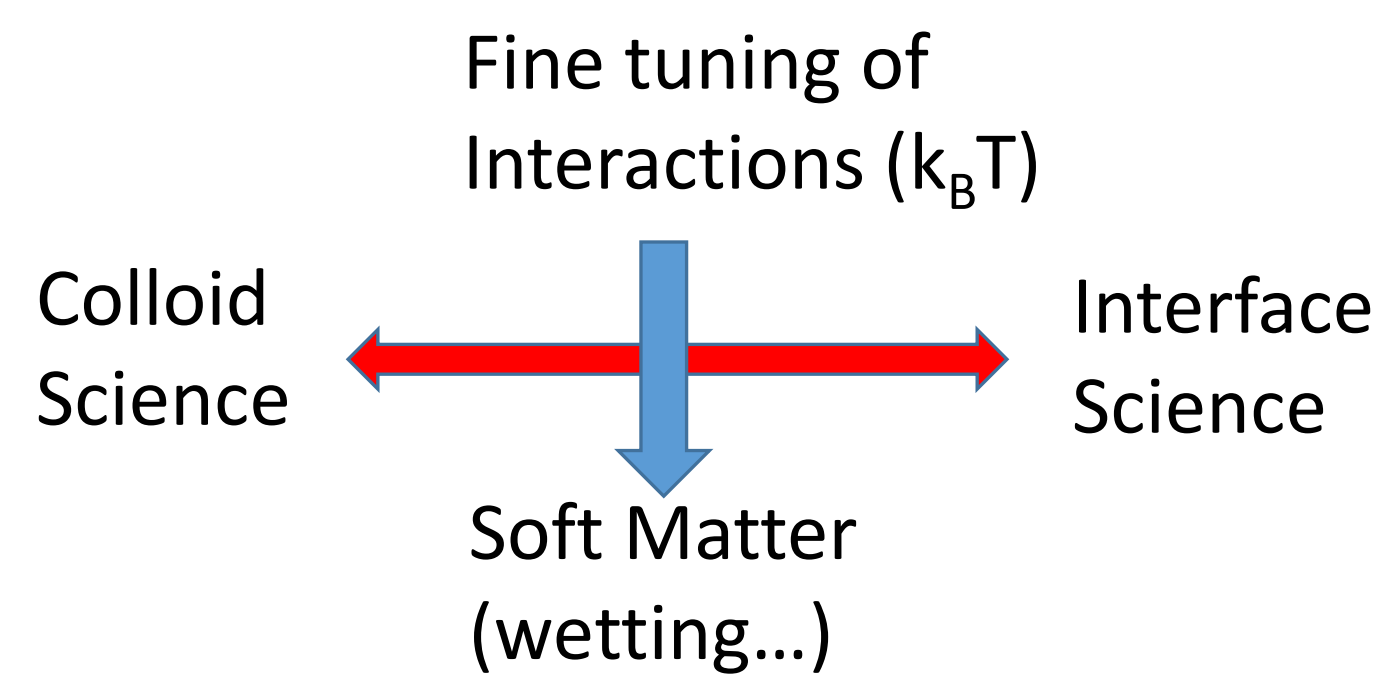

1. Concepts (surface tension, disjoining pressure, adsorption)

2. Wetting (contact angle, line tension, Wenzel and Cassie models)

3. (De)stabilization mechanisms in dispersed systems (gas in liquid:

Foams) 


\section{REFERENCES}

Colloidal Domain

Intermolecular and Surface forces

Physical Chemistry of Surfaces

Capillarity and Wetting Phenomena

The Physics of Foams
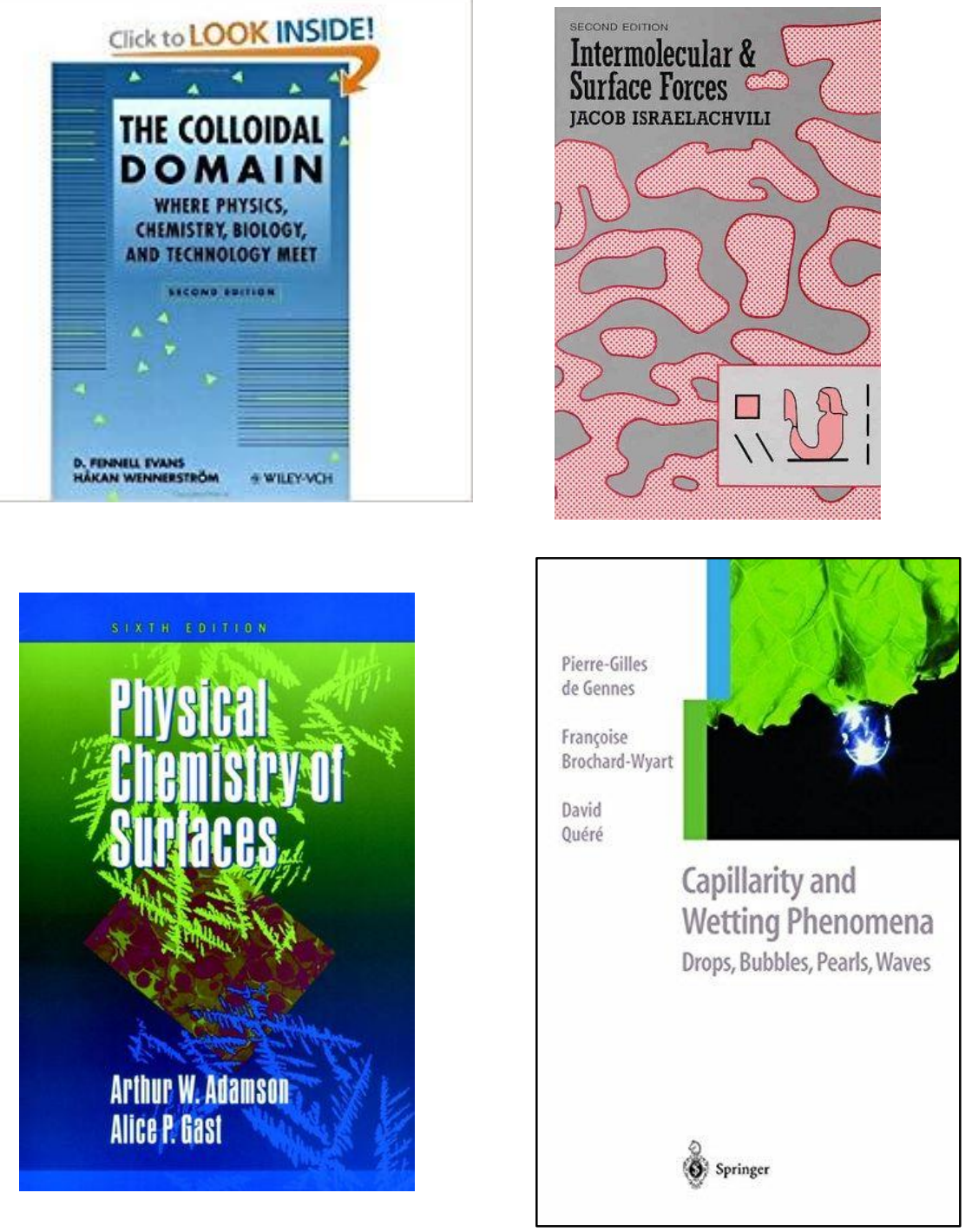

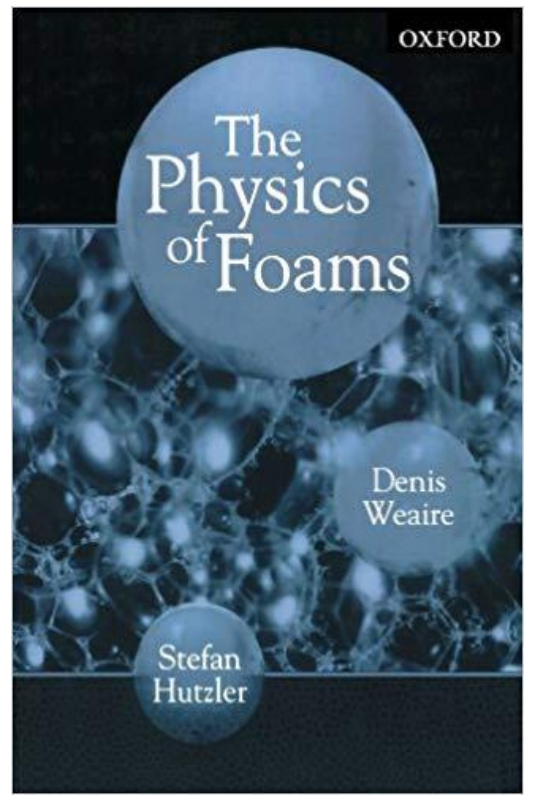

Some slides from Dominque Langevin and Douglas Durian 


\section{Introduction - Wetting}

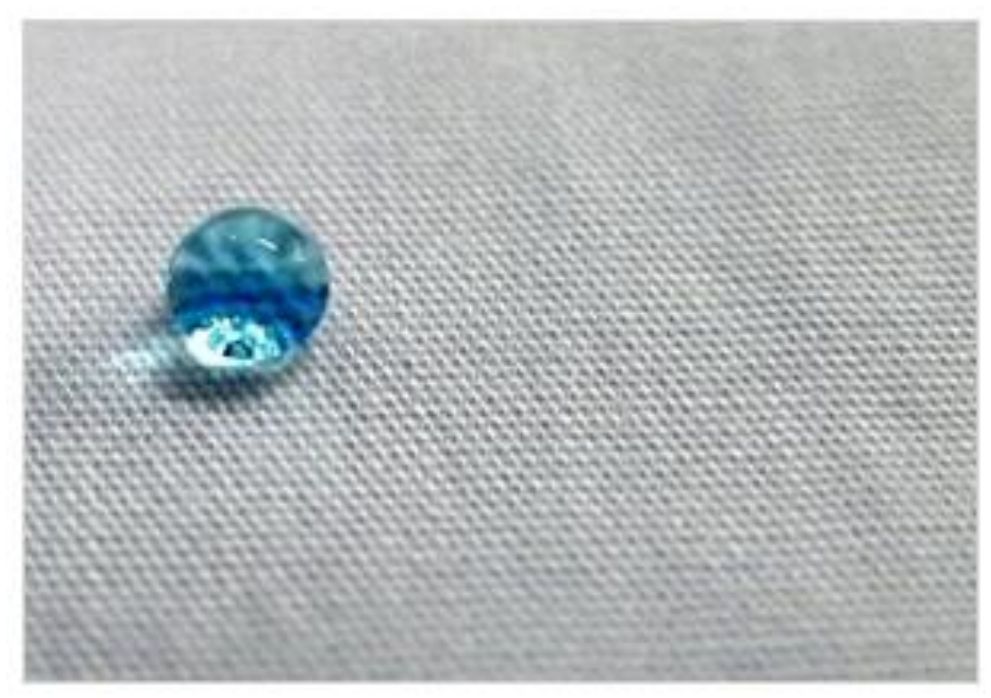

Fig. 1: A water droplet resting on

superhydrophobic cotton.

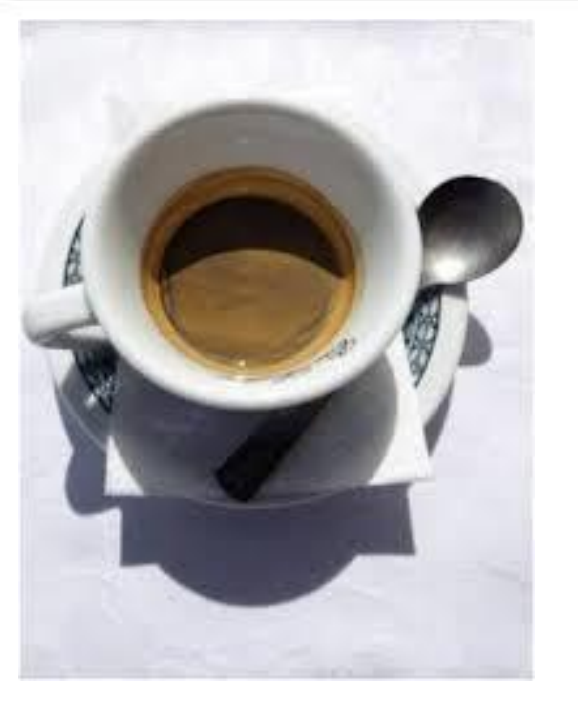

Coffee ring effect

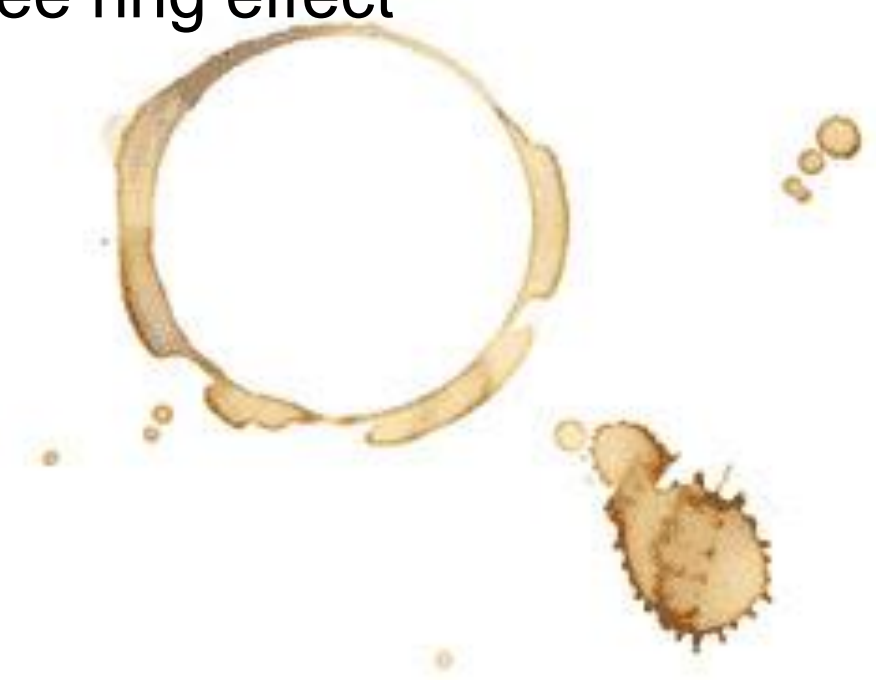

Rain drops on Window

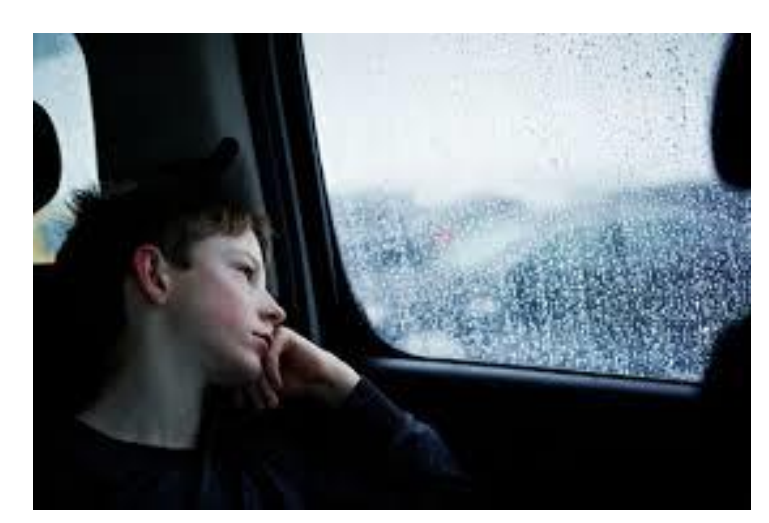

\section{Leidenfoster effect}

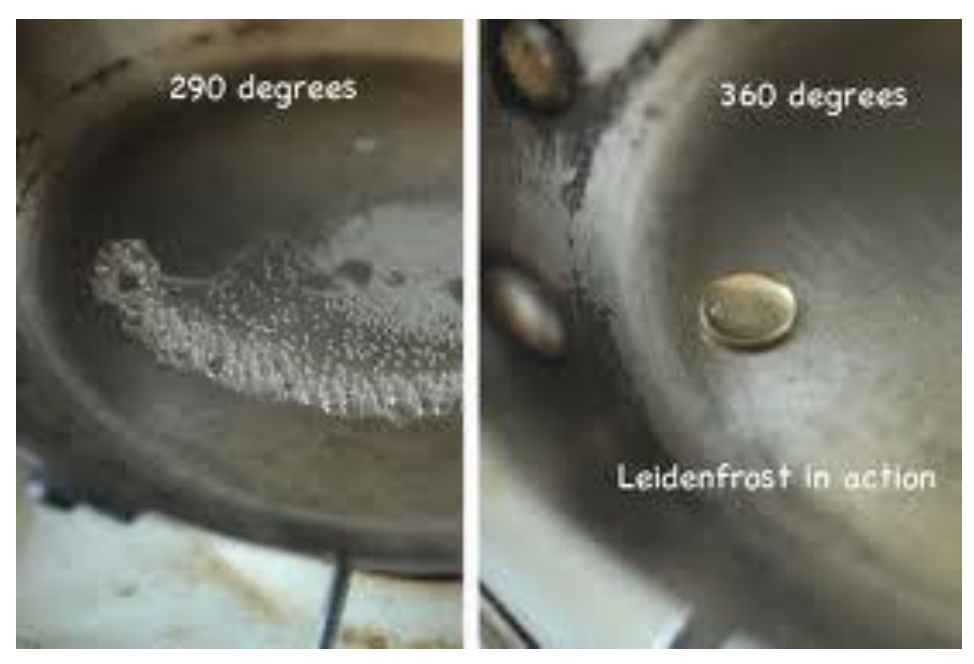




\section{Introduction - Surfaces}

Surface properties of Materials vs. Bulk properties

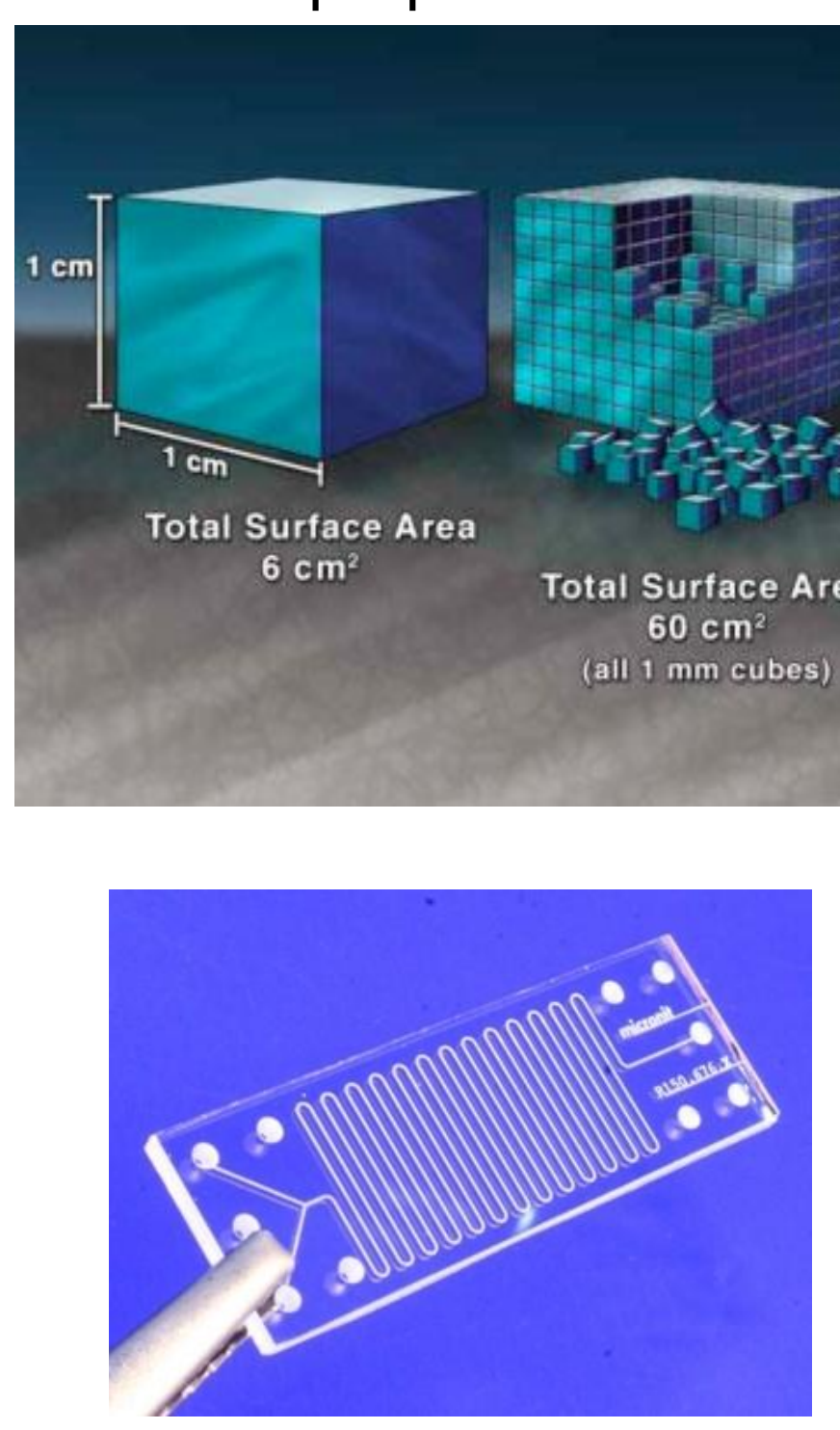

$\mu$-Fluidics

Nano-Fluidics

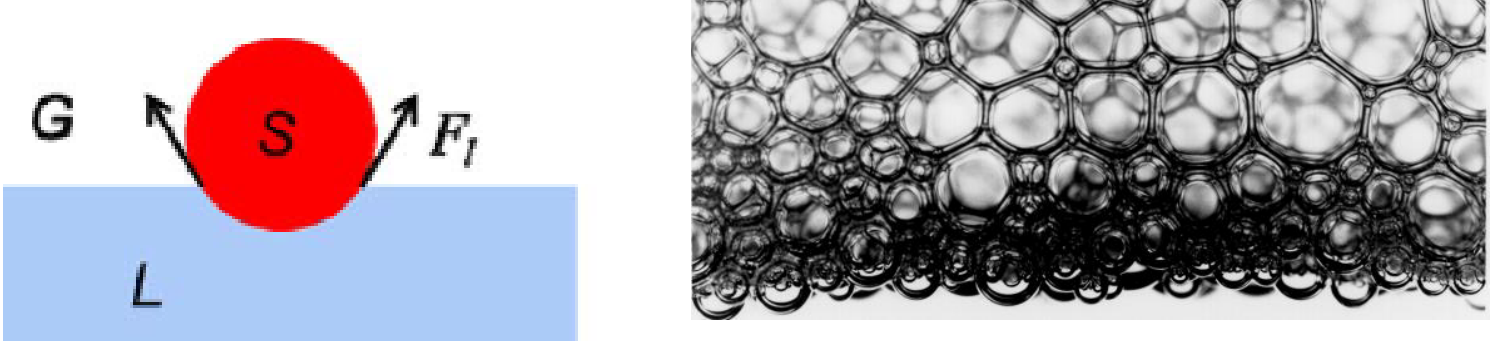

Foams / Emulsions

Particles@Interfaces

Particles@Membranes

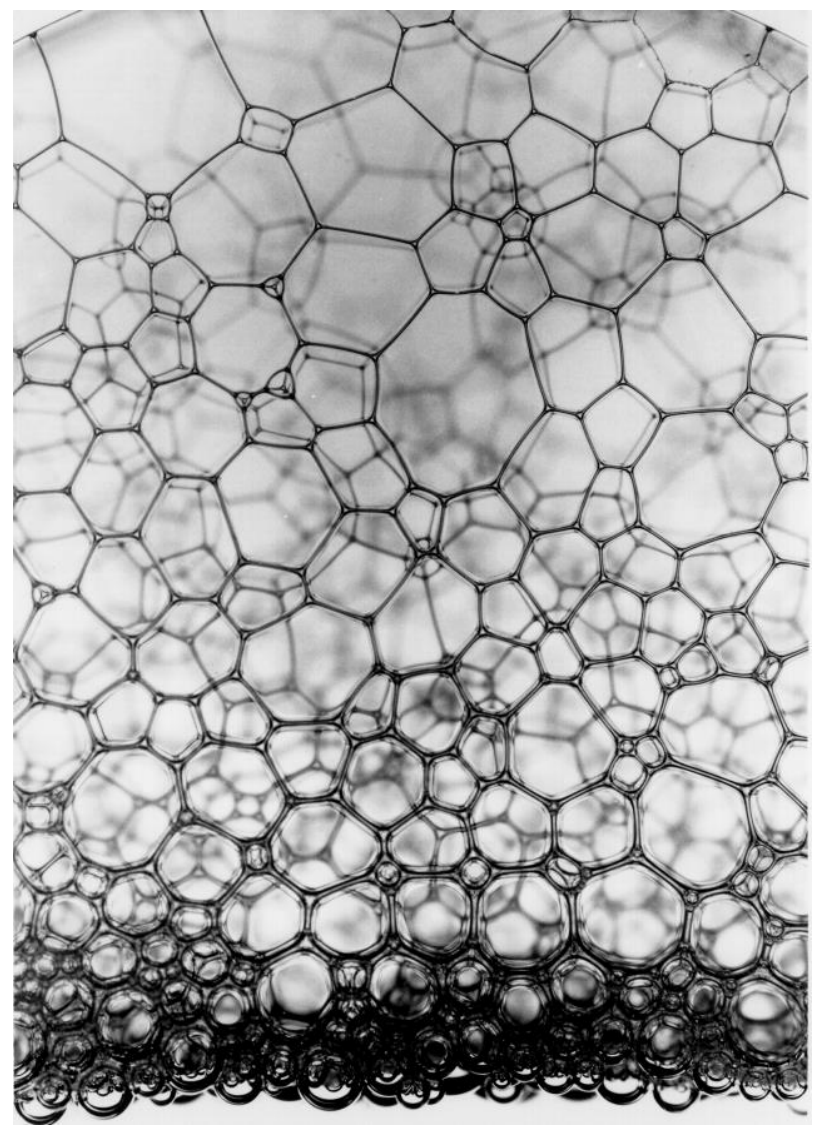




\section{Interface}

- Intrinsic profile

$\mathrm{L}=f($ size of object $)<1 \mathrm{~nm}$

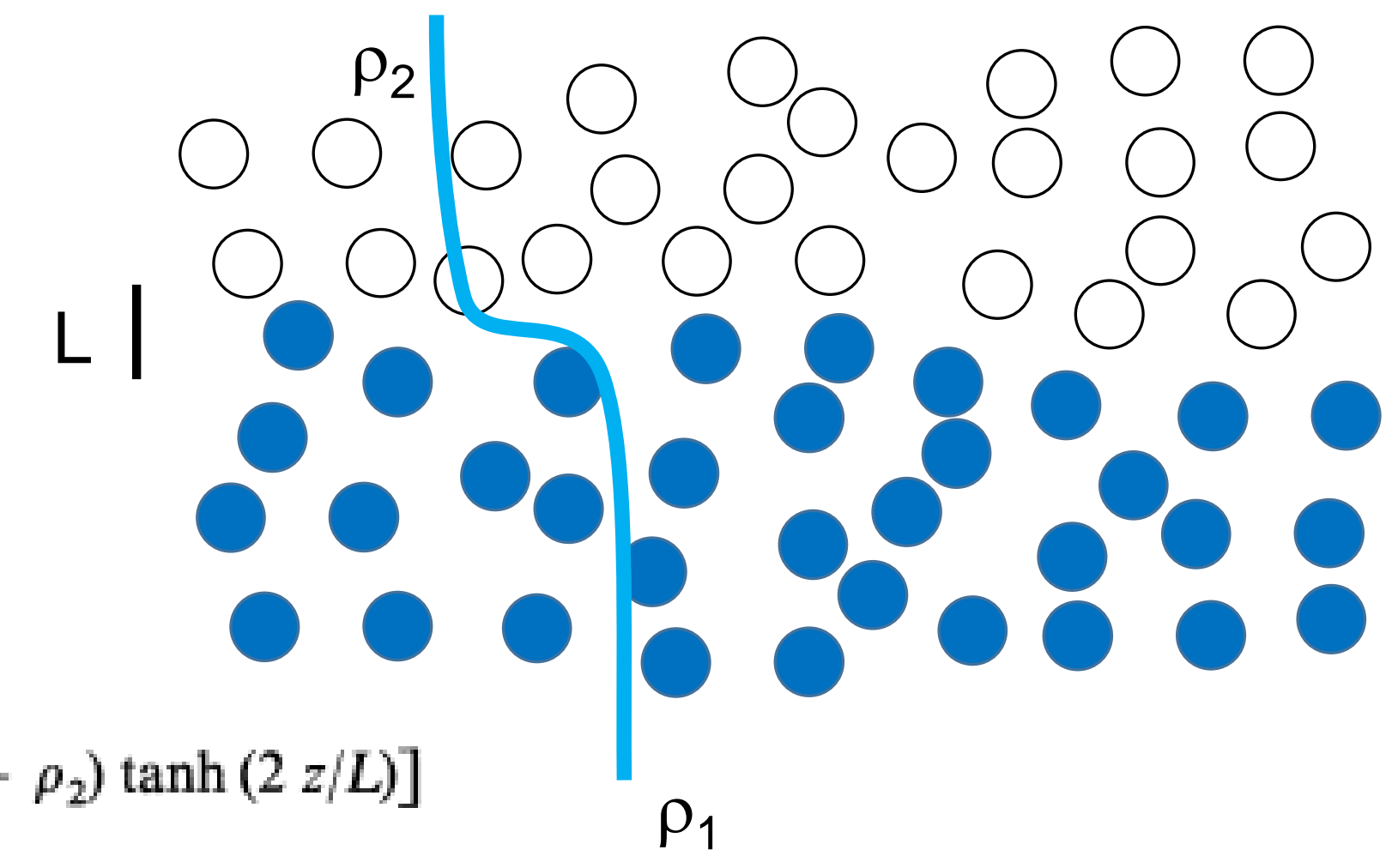

- Surface roughness

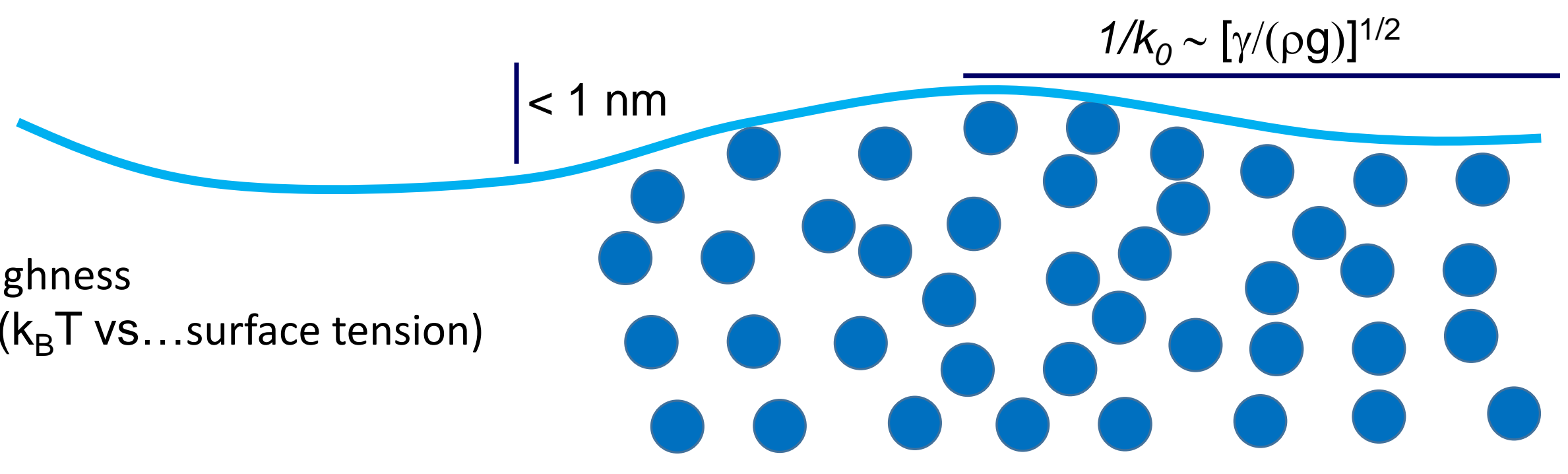

$\mathrm{L}=f\left(\mathrm{k}_{\mathrm{B}} \mathrm{T}\right.$ vs...surface tension $)$ 

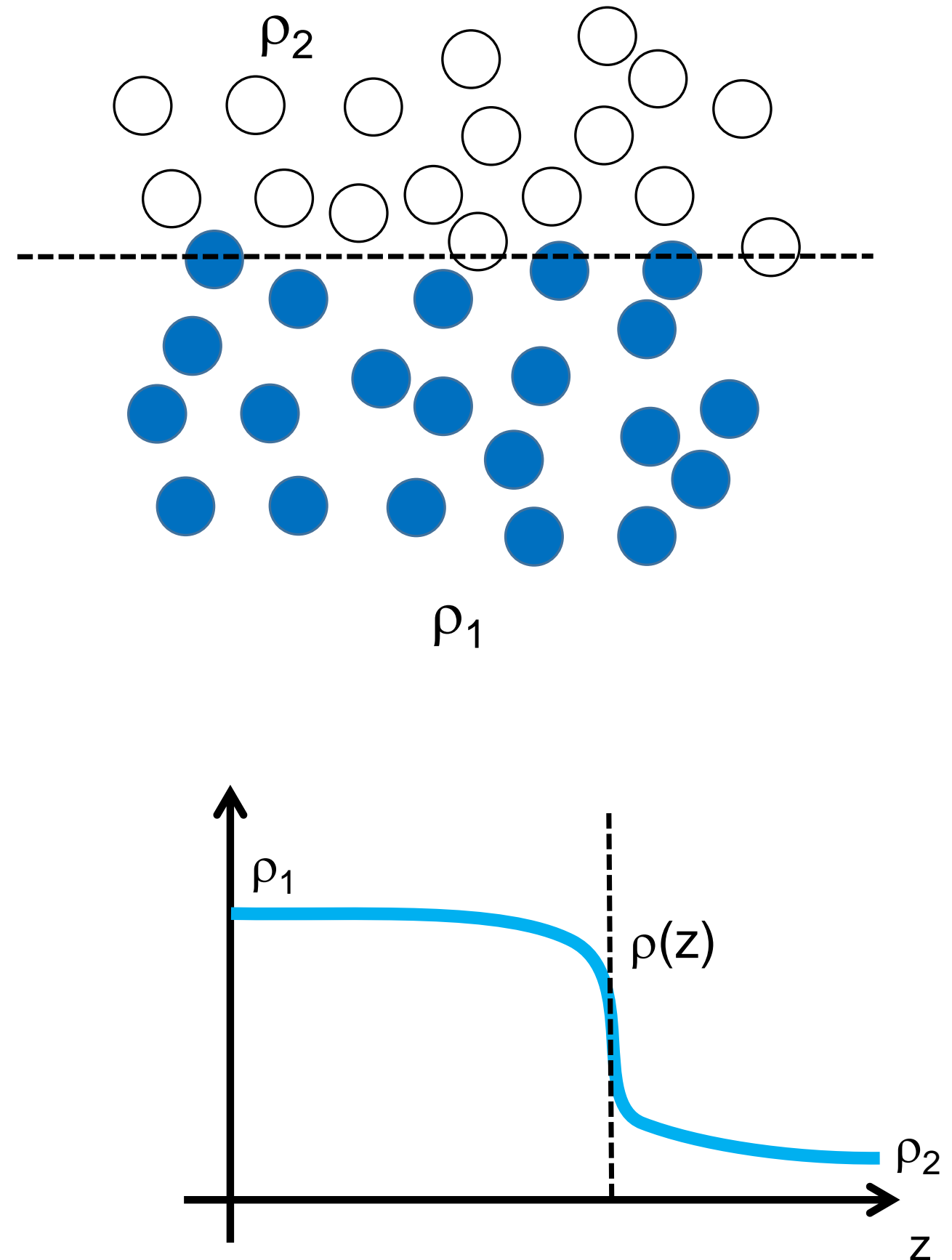

2. Interfacial tension $\gamma$

(cohesive energy)

nearest neighbours exchanges

$$
\begin{aligned}
& E=A \int_{-\infty}^{+\infty} f_{E}(z) d z=E_{1}+E_{2}+\Delta E \\
& \Delta E=\gamma A \quad A \text { is the area }
\end{aligned}
$$

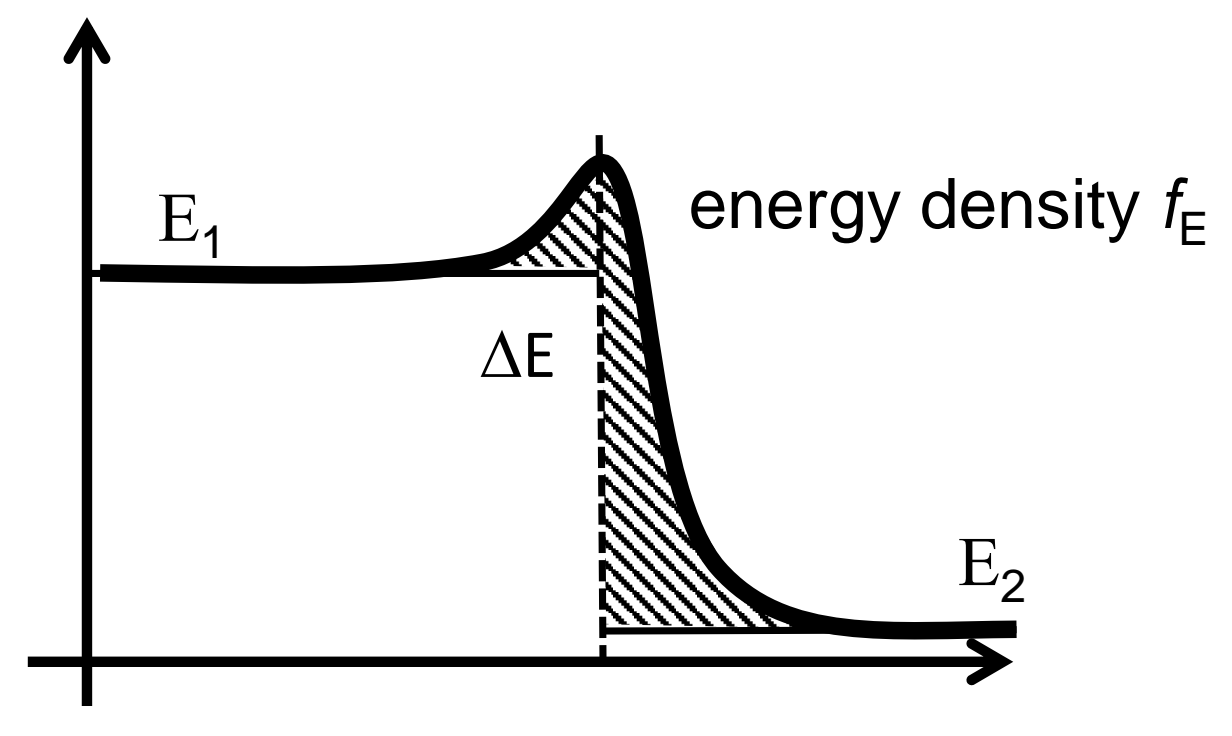


2. Interfacial tension

Force definition $\quad f=\gamma L$

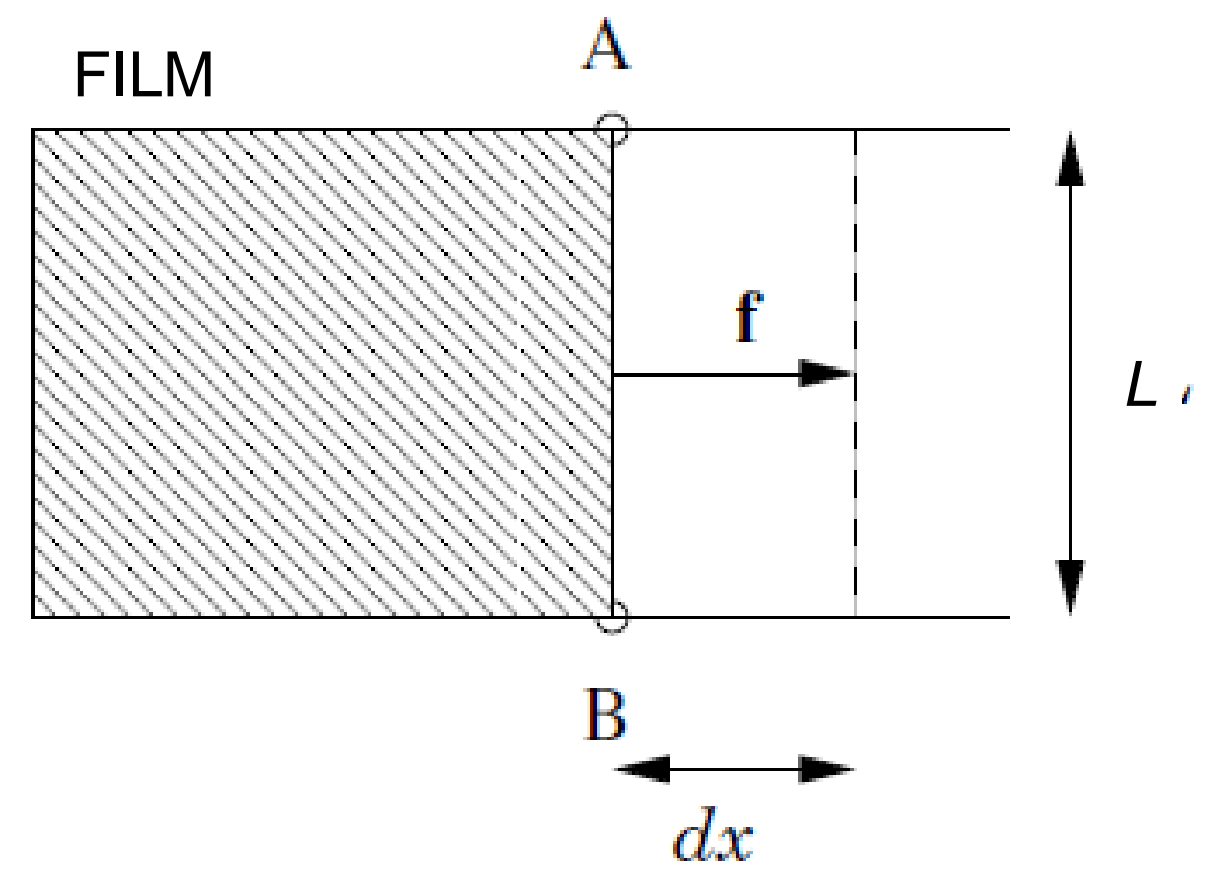

Thermodynamic definition

$$
\begin{aligned}
& d W=f d x=\gamma L d x=\gamma d A \\
& d U=\gamma d A+T d S \\
& \gamma=(d U / d A)_{T}-T(d S / d A)_{T} \\
& d E=d U-T d S-S d T
\end{aligned}
$$$$
\mathrm{d} E=\gamma \mathrm{d} A-S \mathrm{~d} T
$$$$
(\mathrm{d} E / \mathrm{d} A)_{\mathrm{T}}=\gamma
$$$$
\gamma_{i} \text { and } \quad \sigma_{i}=\left(\frac{d E}{d A_{i}}\right)_{T, V, n}
$$ 


\section{Liquid Helium}

Octane

Benzene

Glycol

Water

Silica (SiO2)

Metals (above Tm)

Aluminium

Silver

Iron
(M. Gradzielsky)
$0.2 \mathrm{mN} / \mathrm{m}$

$21 \mathrm{mN} / \mathrm{m}$

$28 \mathrm{mN} / \mathrm{m}$

$48 \mathrm{mN} / \mathrm{m}$

$73 \mathrm{mN} / \mathrm{m}$

$76 \mathrm{mN} / \mathrm{m}$
$700 \mathrm{mN} / \mathrm{m}$

$1000 \mathrm{mN} / \mathrm{m}$ $1500 \mathrm{mN} / \mathrm{m}$ 


\section{Laplace Equation:}

$$
\begin{aligned}
\Delta p & =-\gamma \nabla \cdot \hat{n} \\
& =2 \gamma H \\
& =\gamma\left(\frac{1}{R_{1}}+\frac{1}{R_{2}}\right)
\end{aligned}
$$

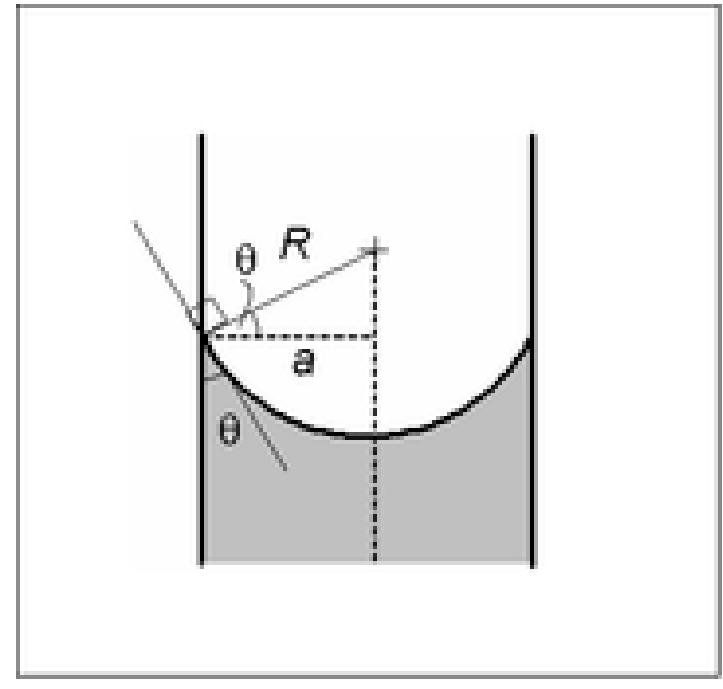

$$
\Delta p=\frac{2 \gamma}{R}
$$

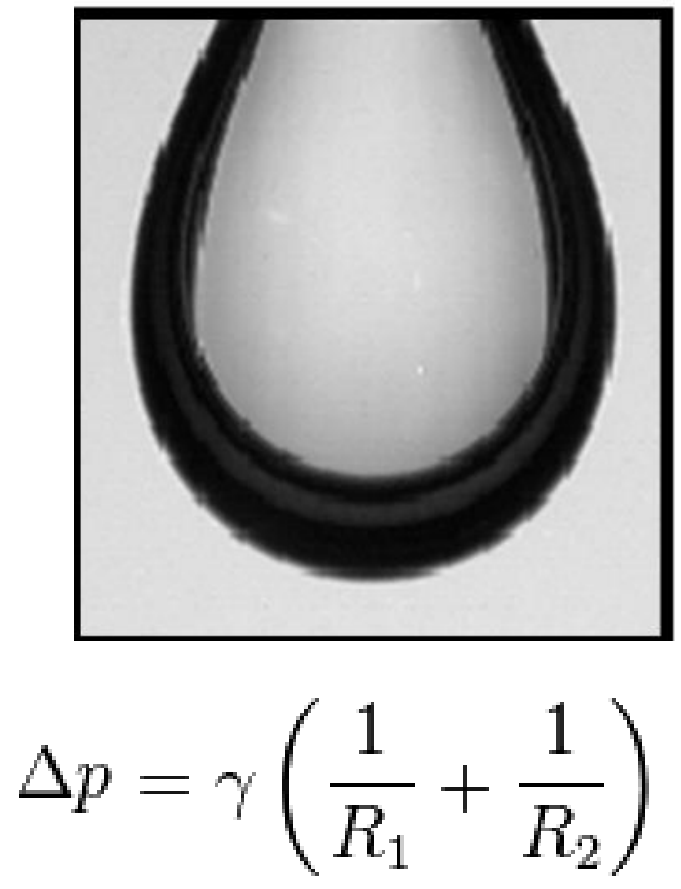

Nanobubble's pressure

$100 \mathrm{~nm}$ in water the extra pressure in the bubble is $1.45 \mathrm{MPa}$ or $\sim 14.3$ atmospheres and for a $10 \mathrm{~nm}$ radius bubble, the extra pressure is $14.5 \mathrm{MPa}$. 


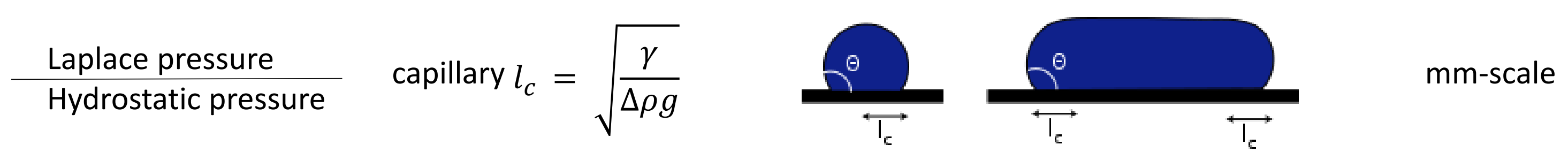

Elastocapillary

$$
l_{E C}=\sqrt{\frac{B}{\gamma}}
$$

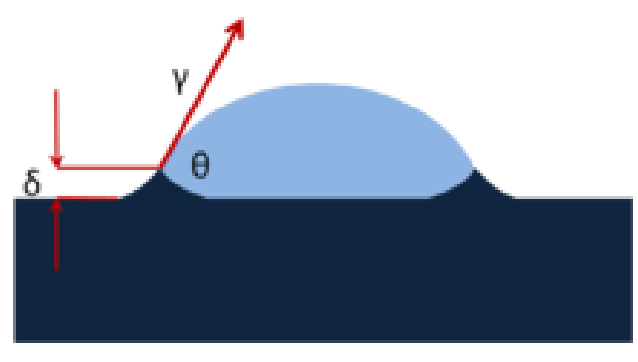

$\mathrm{F}_{\text {vertical }}=\gamma \sin (\theta)=\mathrm{B} \delta$

$B=$ bending elastic modulus

glass ( $\mathrm{B} \sim 700 \mathrm{GPa}$ ), $I_{\mathrm{EC}} \sim 10^{-12} \mathrm{~m}$ - negligible.

PDMS ( $B \sim 300 \mathrm{kPa}$ ), deformation to be $I_{\mathrm{EC}} \sim 10^{-6} \mathrm{~m}$,
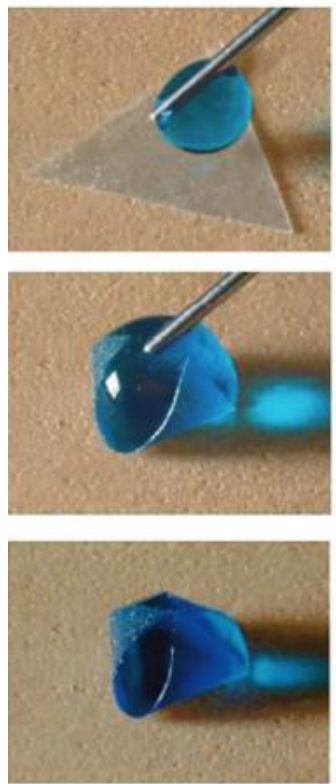


\section{Van der Waals interactions}

Hamaker constants calculated on the basis of the Lifshitz theory

Two media 1 and 2 interacting across a medium 3:

$$
\begin{aligned}
A_{H}= & A_{H, 0}+A_{H, v} \\
A_{H, 0}= & \frac{3}{4} k_{B} T\left(\frac{\varepsilon_{1}-\varepsilon_{3}}{\varepsilon_{1}+\varepsilon_{3}}\right)\left(\frac{\varepsilon_{2}-\varepsilon_{3}}{\varepsilon_{2}+\varepsilon_{3}}\right) \\
& A_{H, v}=\frac{3 h_{P} v_{e}}{8 \sqrt{2}} \frac{\left(n_{1}^{2}-n_{3}^{2}\right)\left(n_{2}^{2}-n_{3}^{2}\right)}{\left(n_{1}^{2}+n_{3}^{2}\right)^{0.5}\left(n_{2}^{2}+n_{3}^{2}\right)^{0.5}\left[\left(n_{1}^{2}+n_{3}^{2}\right)^{0.5}+\left(n_{2}^{2}+n_{3}^{2}\right)^{0.5}\right]}
\end{aligned}
$$

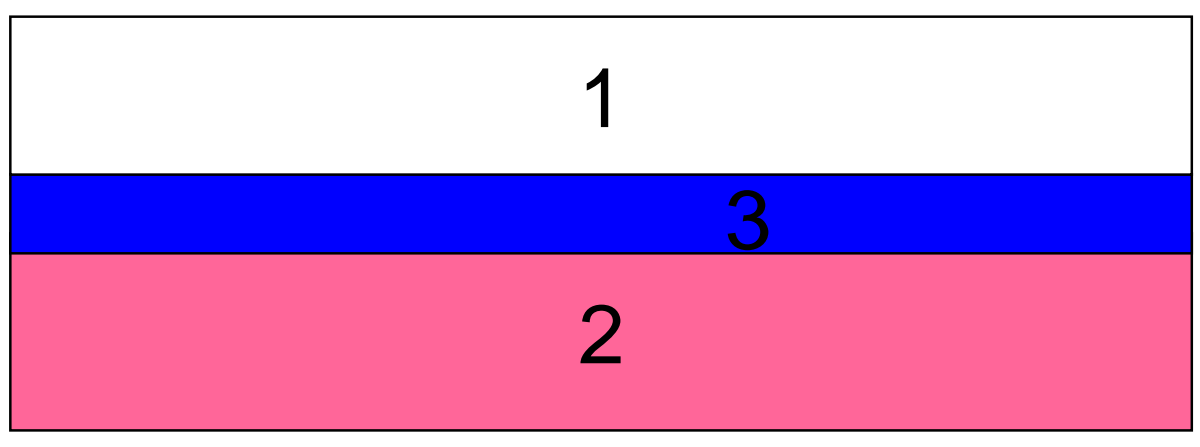

$A_{H, 0}<(3 / 4) k_{B} T$

where $k_{B}$ is the Boltzmann constant, $h_{P}$ is the Planck constant $\left(6.62610^{-34} \mathrm{~J} . \mathrm{s}\right)$ and $v_{e}$ is the main electronic absorption frequency $\left(\approx 310^{15} \mathrm{~s}^{-1}\right)$.

Free energy per unit area between two planar surfaces spaced by a distance $h$ due to Van der Waals long range force is:

$V_{V d W}=-\frac{A_{H}}{12 \pi h^{2}}$

(long range)
$\mathrm{A}_{\mathrm{H}}>0$ attractive

$A_{H}<0$ repulsive
Symmetry $\rightarrow$ Attraction (Foam Lamella) thinning

Asymmetry $\rightarrow$ Repulsion (Wetting on solid) thickening 


\section{Disjoining pressure}

Free energy of a film $L$ on a substrate $S$ (or $G / L / G$ or L1/L2/L1 systems)

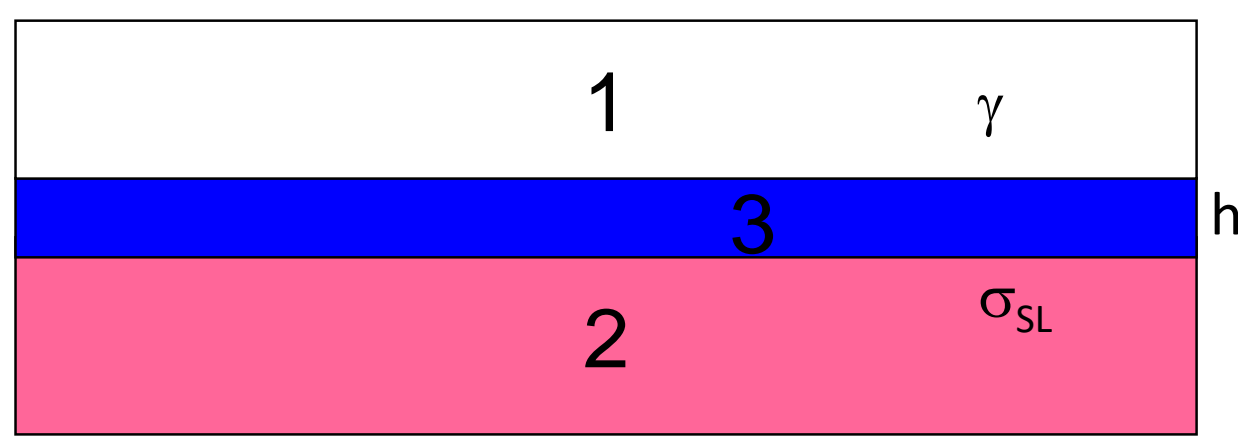

Energy per unit area

$E(h)=\sigma_{S L}+\gamma+P(h)$

Disjoining pressure $\Pi(h)=-\frac{d P}{d h}$

Energy per unit area $\quad V_{V d W}=-\frac{A_{H}}{12 \pi h^{2}}$

\section{$1-30 \mathrm{~nm}$}

DLVO

$V_{E D L}=\frac{64 k_{B} T c_{\text {ion, bulk }}}{\kappa}\left(\tanh \frac{e \psi_{0}}{4 k_{B} T}\right)^{2} \exp (-\kappa h)$

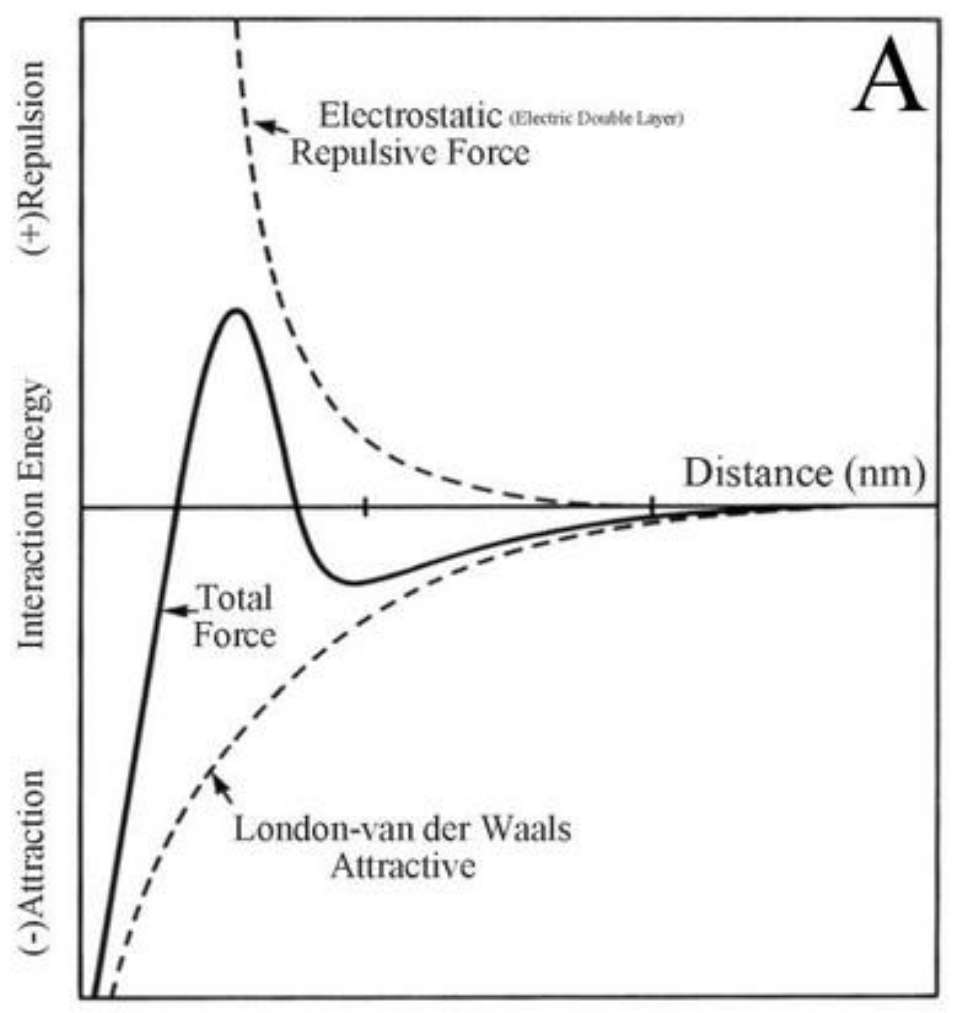


Up to now pure $(L, S, G)$ systems, now binary or more systems

bulk $\quad d U=T \cdot d S-p \cdot d V+\sum_{i} \mu_{i} \cdot d n_{i}$

Surface (interface):

$$
\begin{aligned}
n_{i, s} & =n_{i}-n_{i, \alpha, b}-n_{i, \beta, b} \quad \text { (interphase) } \\
V_{s} & =V-V_{\alpha, b}-V_{\beta, b} \\
U_{s} & =U-U_{\alpha, b}-U_{\beta, b} \\
S_{S} & =S-S_{\alpha, b}-S_{\beta, b} \\
& d U_{S}=T d S_{S}-p d V_{S}+\sigma d A+\sum_{i} \mu_{i} d n_{i, S}
\end{aligned}
$$

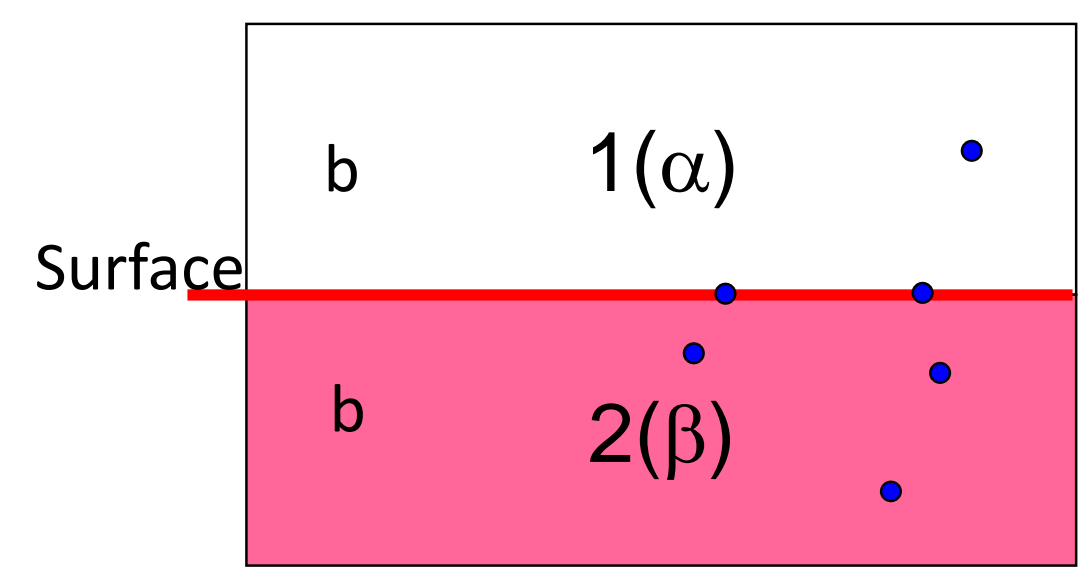

Advances in Colloid and Interface Science

Volume 256, June 2018, Pages 163-192

Historical perspective

The chemical (not mechanical) paradigm of thermodynamics of colloid and interface science

"the fundamental equation of colloid and interface science" 


\section{Adsorption}

(M. Gradzielsky)

2

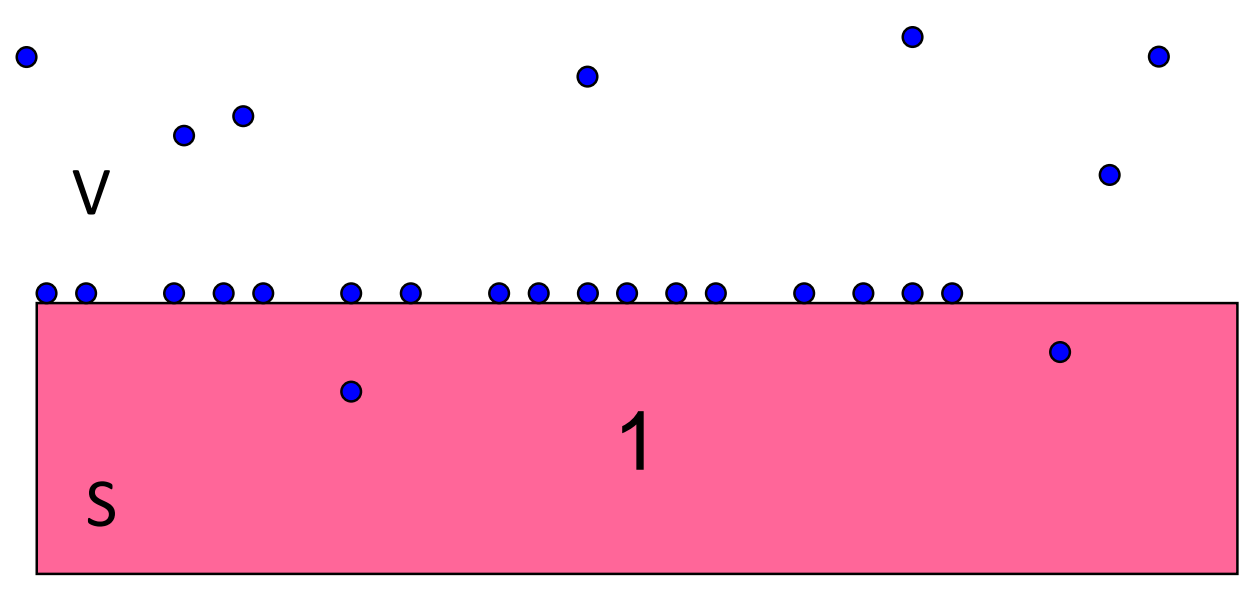

$d \sigma_{S V}=-\Gamma d \mu+S d T$

(Gibbs adsorption)

$\left(d \sigma_{S V} / d \mu\right)_{T}=-\Gamma$

$\mu$ is the chemical potential

$\mathrm{d} \mu=\mathrm{k}_{\mathrm{B}} \top \mathrm{dln}(\mathrm{c})$

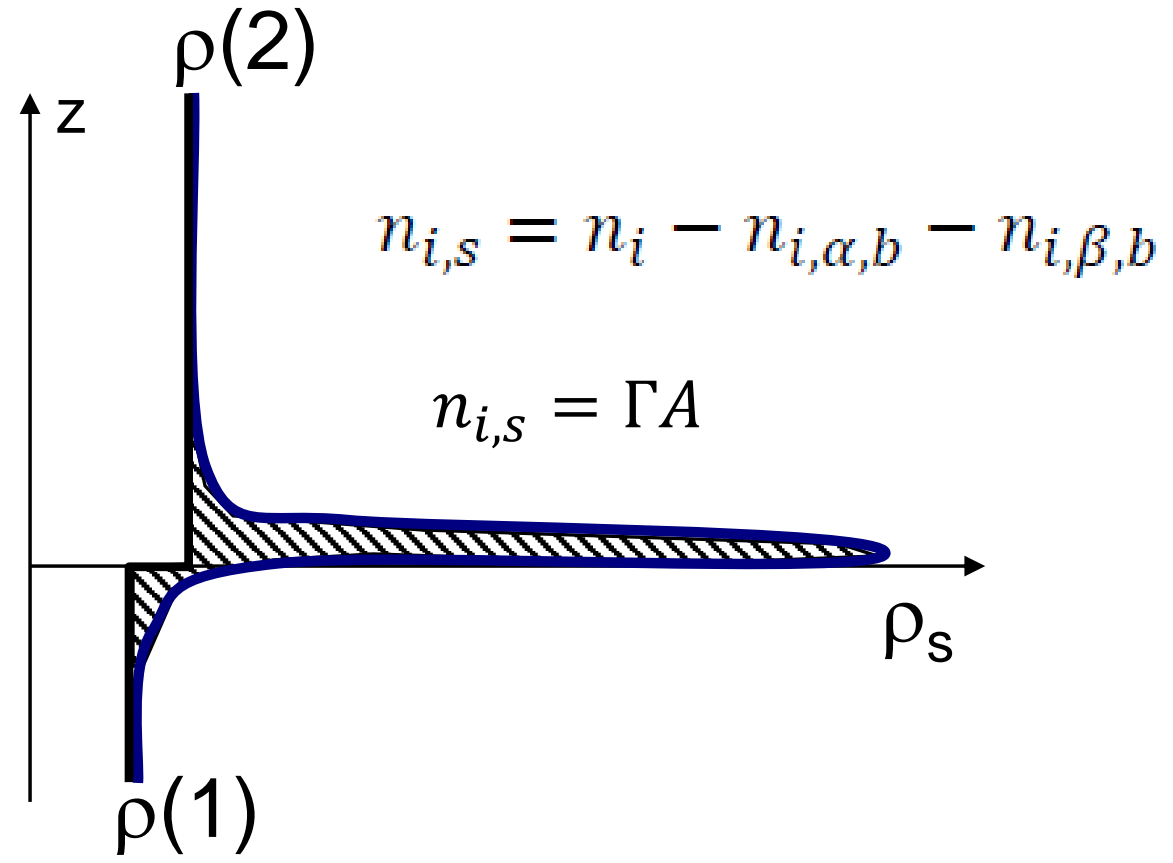

$\Gamma$ is the surface excess conc.

$$
\Gamma=-1 / \mathrm{k}_{\mathrm{B}} \mathrm{T}\left(\mathrm{d} \sigma_{\mathrm{SV}} / \mathrm{dlnc}\right)_{\mathrm{T}}
$$

When surface active species interact with each others this equation is not valid anymore 


\section{Dimensions $\rightarrow$ 2Dimensions $\quad$ Langmuir monolayer}

$$
(\mathrm{P}, V, \mathrm{C}) \rightarrow(\Delta \gamma, \mathrm{A}, \Gamma)
$$

Ideal gas Law

$P=\mathrm{c} \mathrm{RT}$

Bulk Modulus

$K=-V \mathrm{~d} P / \mathrm{d} V$

Rheology $E^{*}, G^{*}$

viscosity

Phase diagrams

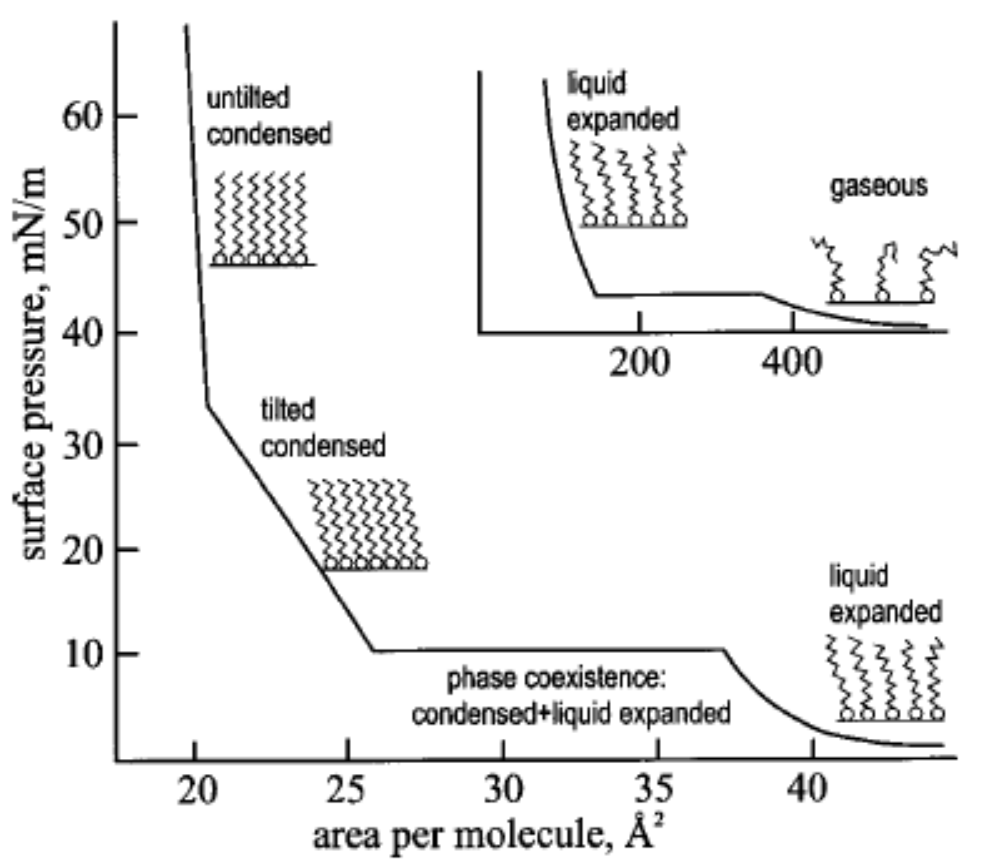

Two dimensional ideal gas

$$
\pi=\gamma_{0}-\gamma=\mathrm{k}_{\mathrm{B}} \mathrm{T} \Gamma
$$

$\pi$ is called surface pressure

$\Gamma$ is called surface conc.

$$
K_{\mathrm{s}}=-A \mathrm{~d} \pi / \mathrm{d} A
$$

Rheology $E_{s}^{*}, G_{s}^{*}$

Surface viscosity

Surface phase diagrams (membranes, R. Winter) 


\section{Summary Part 1: Concepts}

- Interface

- Interface tension

- Capillarity

- Disjoining Pressure (VdW, EDL, DLVO + ....)

- Adsorption

- 3D to 2D concepts

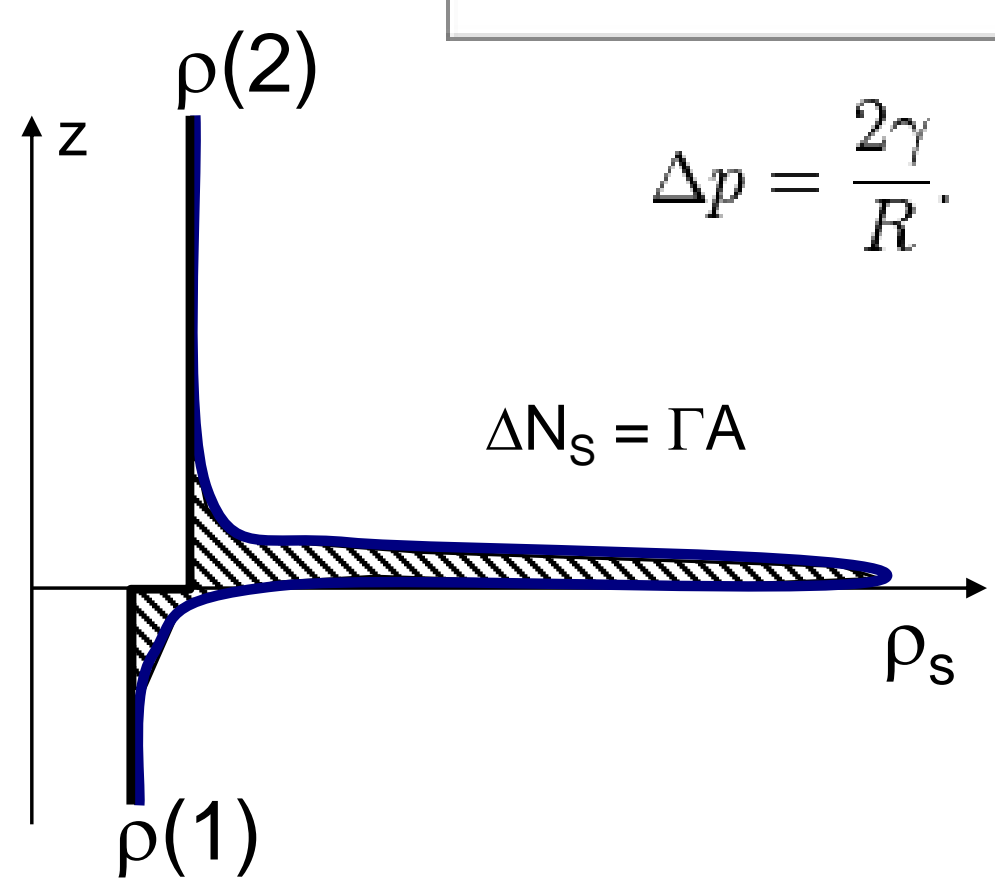

$\Gamma$ is the surface excess conc. 
Fine tuning of

interactions

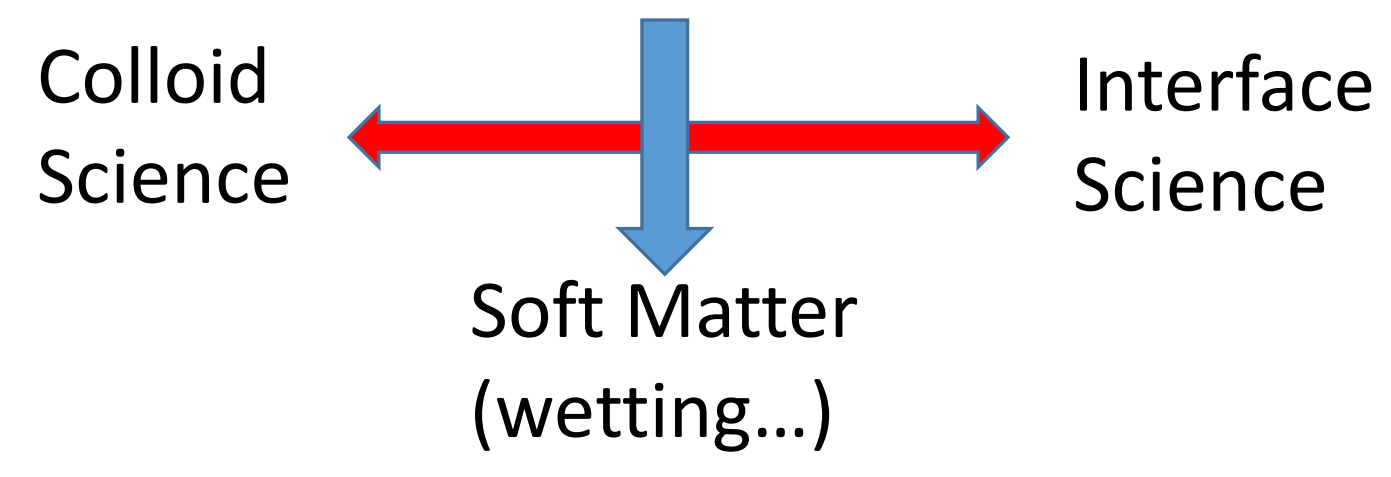

1. Concepts (surface tension, disjoining pressure, adsorption)

2. Wetting (contact angle, line tension, Wenzel and Cassie models)

3. (De)stabilization mechanisms in dispersed systems (gas in liquid: Foams) 
3 Phases, 3 Interfaces, 1 contact line (triple line)

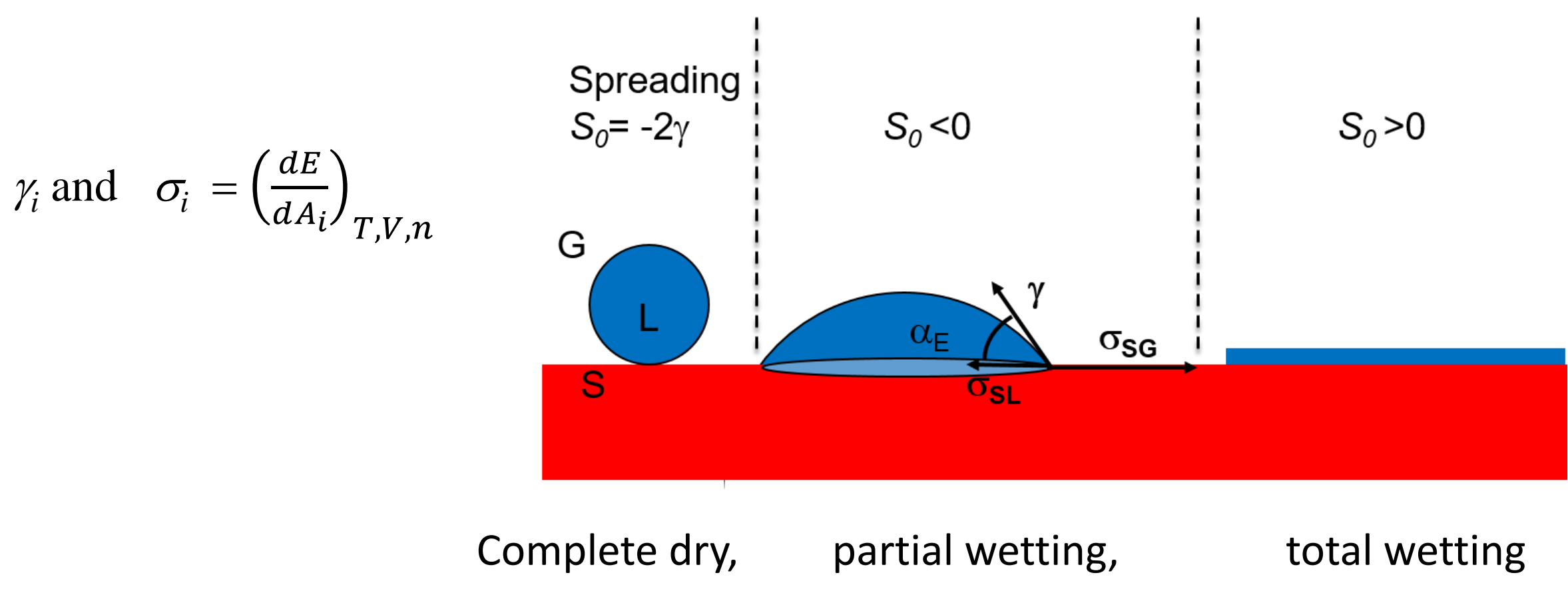

$S_{0}=\sigma_{S G}-\sigma_{S L}-\gamma$, initial (or dry) spreading coefficient

$\begin{array}{lll}\text { Young equation } \quad \cos \alpha_{E}=\left(\sigma_{S G}-\sigma_{S L}\right) / \gamma . & \text { (Horizontal force balance) }\end{array}$ 
a) Liquid drop on planar solid

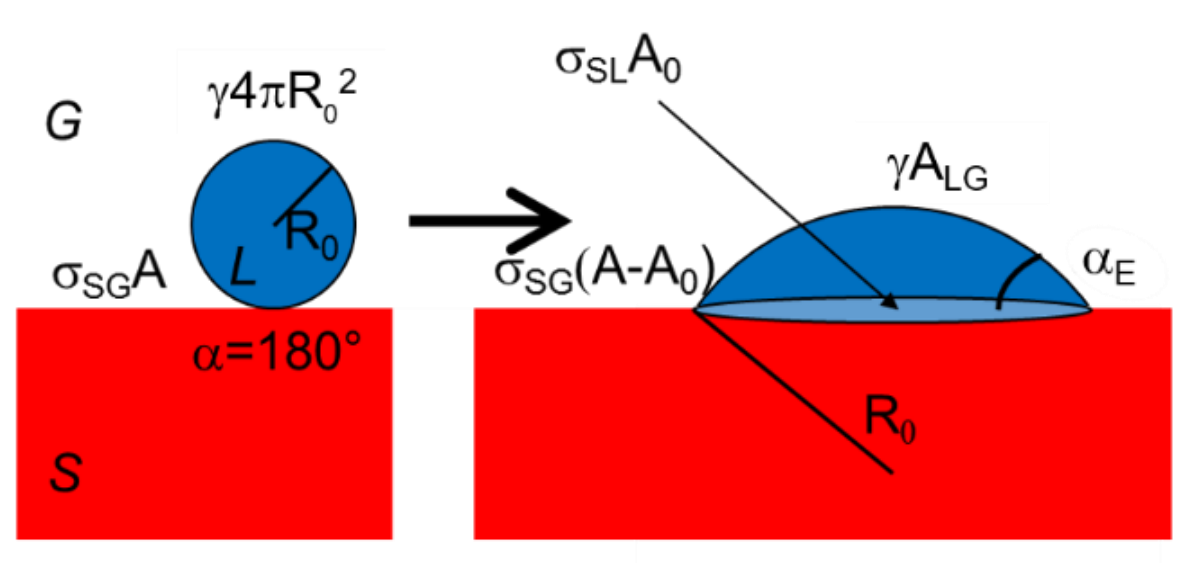

Minimum in free Energy $E(\alpha)$ :

$$
\text { Young Equation } \quad \cos \alpha_{E}=\left(\sigma_{S G}-\sigma_{S L}\right) / \gamma \text {. }
$$

$$
E(\alpha)=\sigma_{S G} A-\sigma_{S G} A_{0}+\sigma_{S L} A_{0}+\gamma A_{L G} .
$$

Constant Volume (area is changing)

$$
\begin{aligned}
& A_{0}=\pi R_{0}(\alpha)^{2} \sin ^{2} \alpha . \\
& A_{L G}=2 \pi R_{0}(\alpha)^{2}(1-\cos \alpha) \\
& R_{0}(\alpha)=V^{\frac{1}{3}}\left[\frac{\pi}{3}\left(2-3 \cos \alpha+\cos ^{3} \alpha\right)\right]^{-\frac{1}{3}}
\end{aligned}
$$


b) Solid particle on liquid surface

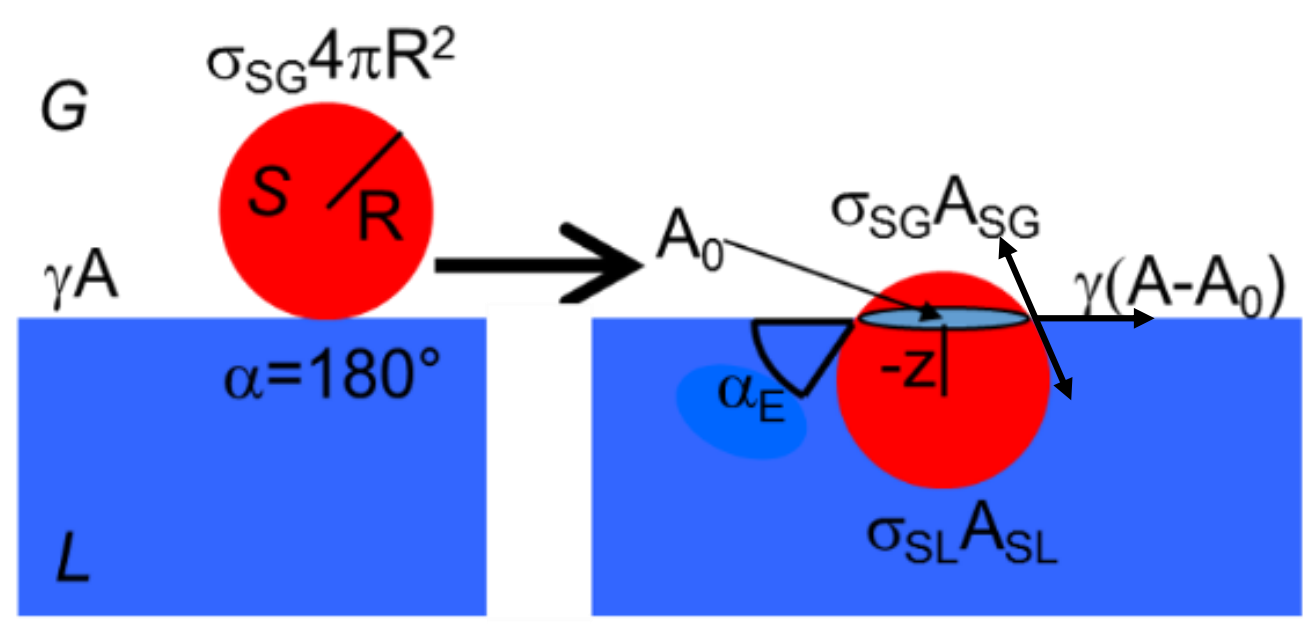

Particle keeps constant Volume and Area

$$
\begin{aligned}
& E(\alpha)=\gamma A+2 \pi R^{2}\left[\sigma_{S G}(1-\cos \alpha)+\sigma_{S L}(1+\cos \alpha)-\frac{1}{2} \gamma \sin ^{2} \alpha\right] . \\
& \left(\sigma_{S G}-\sigma_{S L}\right) / \gamma=-z_{0} / R=\cos \alpha_{E} .
\end{aligned}
$$


The imbalance due to $\alpha \neq \alpha_{E}$ produces a force $F_{l}=-\frac{d E}{d s}$, where $s$ represents the displacement of the contact line on the solid

$F_{l}(\alpha)=2 \pi R_{d} \gamma\left(\cos \alpha_{E}-\cos \alpha\right)=2 \pi R_{0}(\alpha) \sin \alpha \gamma\left(\cos \alpha_{E}-\cos \alpha\right)$

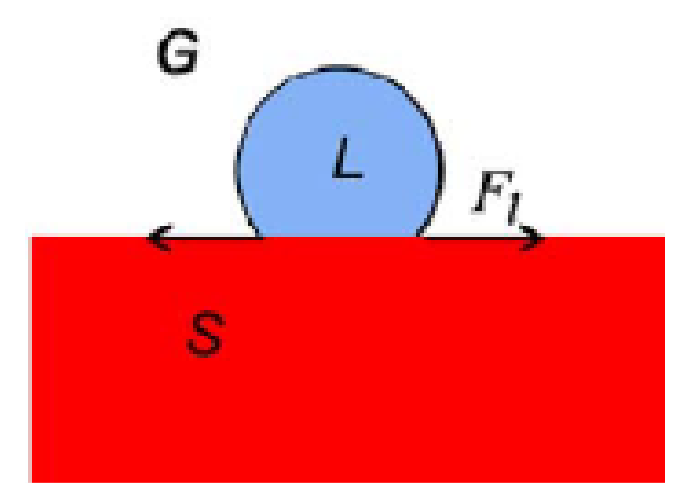

Self-running droplet

For a spherical particle, the displacement of the contact line is the longitudinal coordinate $s=R \theta$ ( $\theta$ is the polar angle in spherical coordinates.

$F_{l}(\alpha)=2 \pi R \sin \alpha \gamma\left(\cos \alpha_{E}-\cos \alpha\right)$

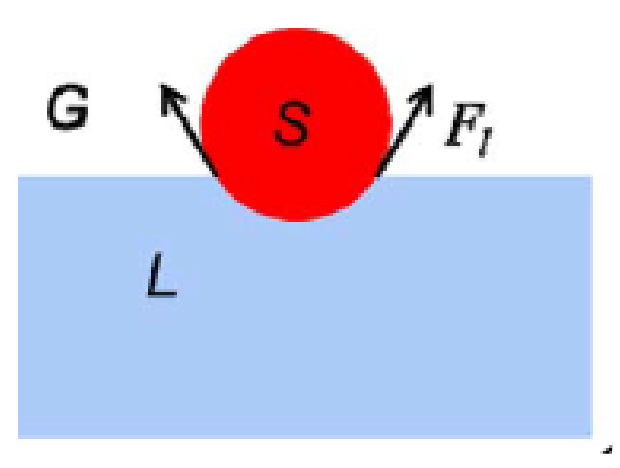


Free Energy and Force on the line
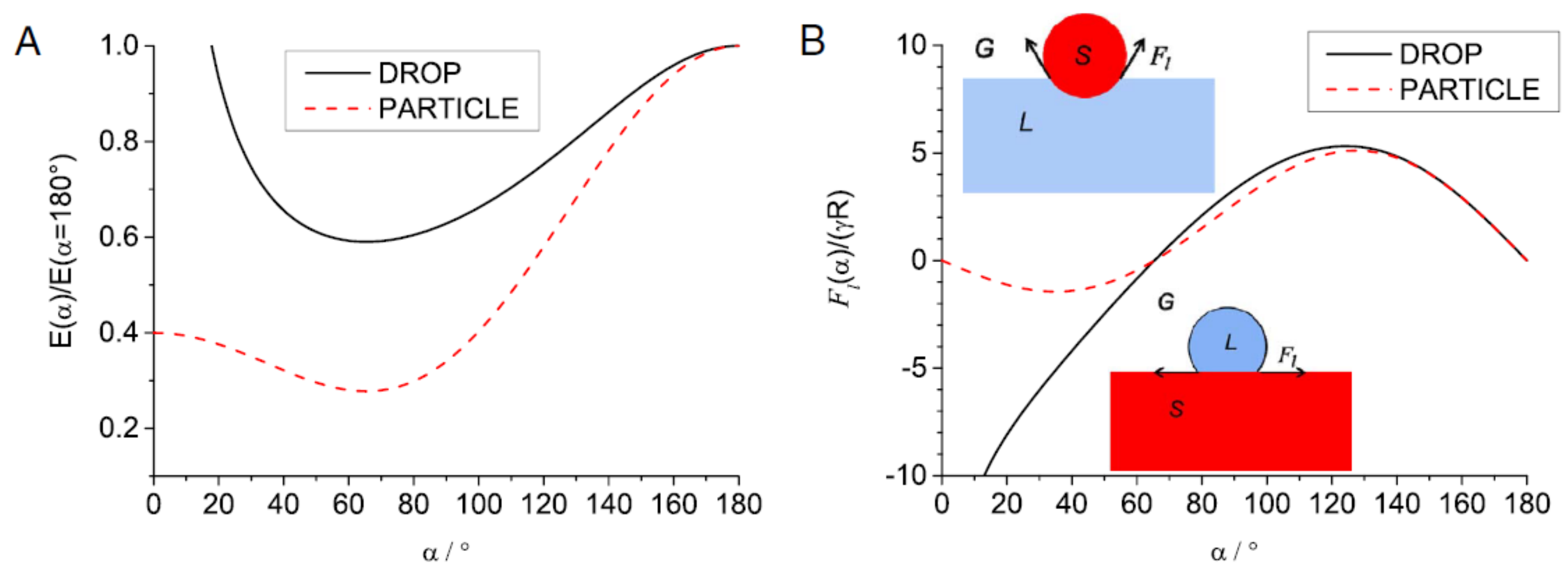

PARTICLE: $\begin{aligned} & R=1 \mu \mathrm{m} \text { silica/water/air , } E=10^{6} \ldots 10^{7} \mathrm{k}_{\mathrm{B}} \mathrm{T} \\ & \mathrm{R}=1 \mathrm{~nm} \text { silica/water/air , } E=1 \ldots 10 \mathrm{k}_{\mathrm{B}} \mathrm{T}\end{aligned}$

Stocco, A. Nobili, M. Advances in Colloid and Interface Science 2017 


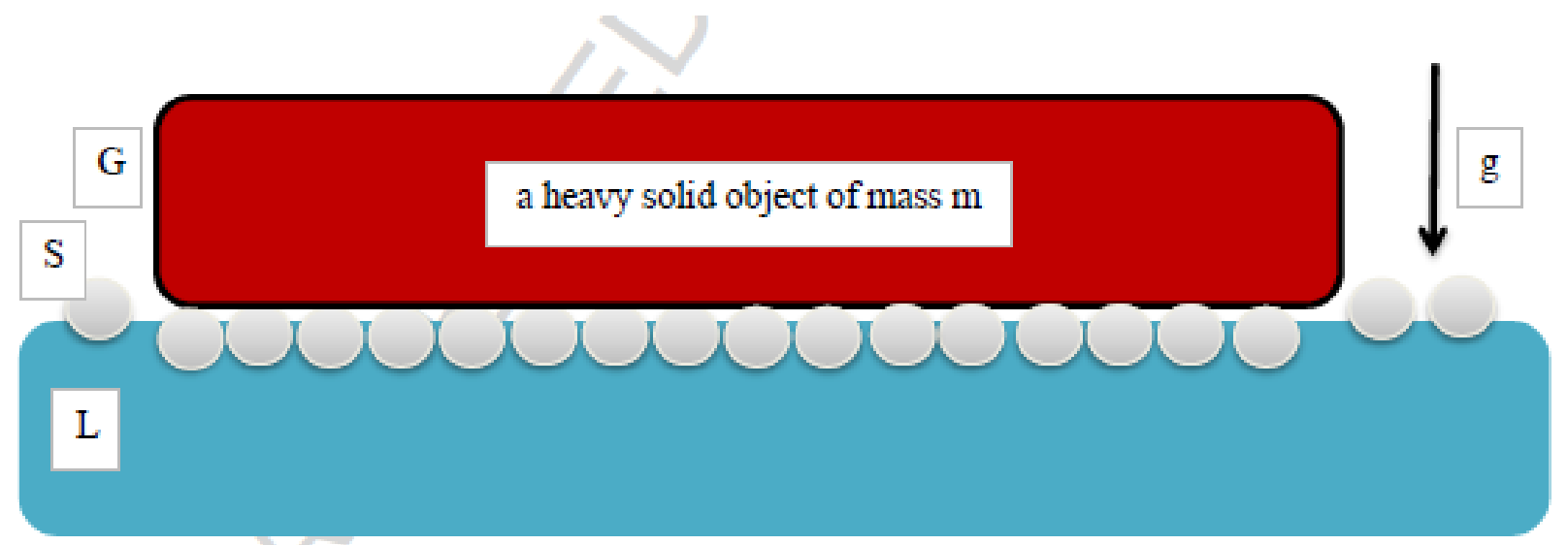

Historical perspective

The chemical (not mechanical) paradigm of thermodynamics of colloid and interface science George Kaptay四

\section{It was not a miracle!}

Fig.6 A large heavy solid object floating on a liquid surface, separated by a large amount of small solid particles at the liquid/gas interface

cannot hold any weight. Now, let us substitute easily accessible parameters into Eq.(6i): $r=1$ $\mu \mathrm{m}, \mathrm{f}=0.8, \mathrm{~g}=9.81 \mathrm{~m} / \mathrm{s}^{2}, \sigma_{\mathrm{L} / \mathrm{G}}=0.07 \mathrm{~J} / \mathrm{m}^{2}, \Theta=60^{\circ}$, resulting to $\left(\frac{m \cdot g}{A}\right)_{\max }=56 \mathrm{kN} / \mathrm{m}^{2}$ (note: area of a sandal of an adult male is about $300 \mathrm{~cm}^{2}=0,03 \mathrm{~m}^{2}$. Multiplying the two latter values and dividing the result by the value of $g$, the maximum mass of the adult male in sandals hold by the above force is $171 \mathrm{~kg}$. So, if still water is covered by a large amount of micron-sized particles, it can easily hold an adult male in a sandal in a way that nor the sandal neither the foot of the male becomes wet (see the Bible claiming Jesus walking on water).

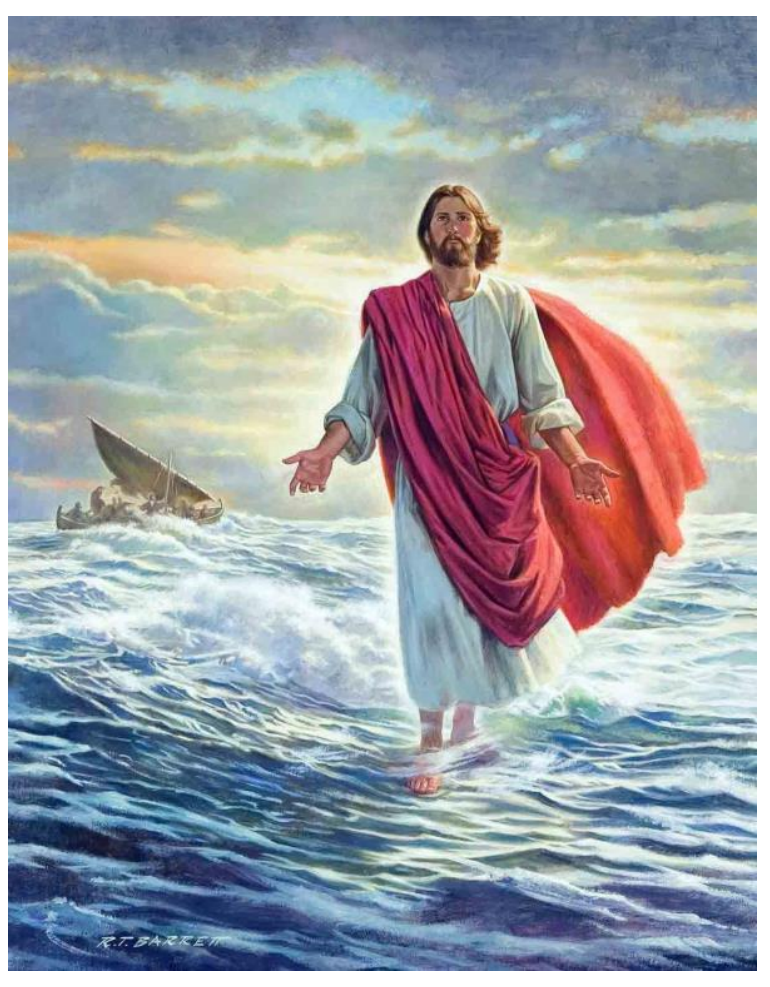


7. Line tension $k$ (important for bilayers, membranes...) for macroscopic drops

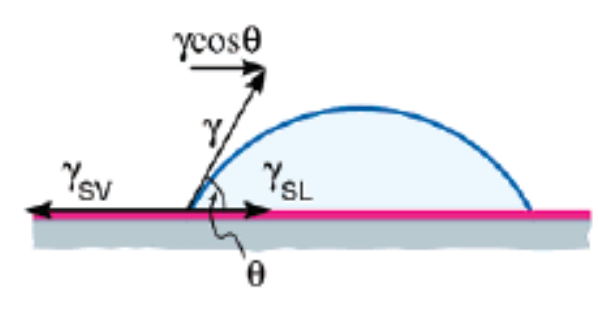

$\cos \alpha_{e q}=\left(\sigma_{S G}-\sigma_{S L}\right) / \gamma$

$$
E(\alpha)=\sigma_{S G} A-\sigma_{S G} A_{0}+\sigma_{S L} A_{0}+\gamma A_{L G}+k 2 \pi R_{0}(\alpha) \sin \alpha .
$$

$\cos \mathcal{A}_{e q}=\cos \alpha_{e q}-\frac{k}{\gamma R_{0}(\alpha)} \frac{1}{\sin \mathcal{A}_{e q}} \quad$ (drop)

$$
E(\alpha)=\gamma A+2 \pi R^{2}\left[\sigma_{S G}(1-\cos \alpha)+\sigma_{S L}(1+\cos \alpha)-\frac{1}{2} \gamma \sin ^{2} \alpha+\frac{k}{R} \sin \alpha\right]
$$

$\cos \mathcal{A}_{e q}=\cos \alpha_{e q}+\frac{k}{\gamma R} \frac{\cos \mathcal{A}_{e q}}{\sin \mathcal{A}_{e q}} \quad$ (particle)

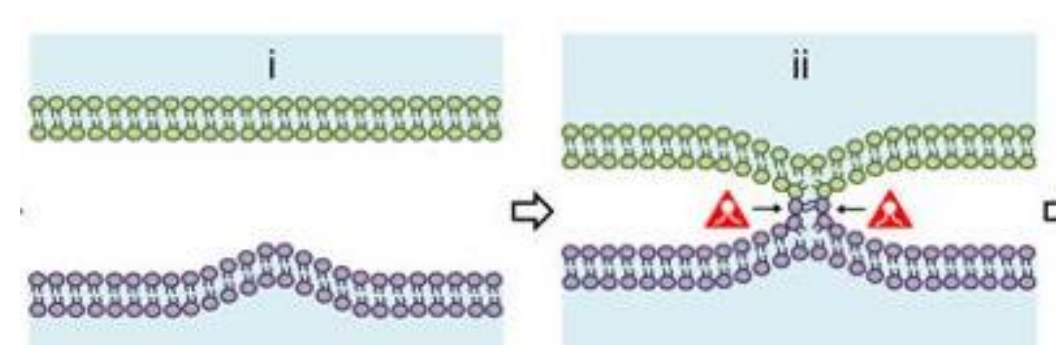

iii

iv

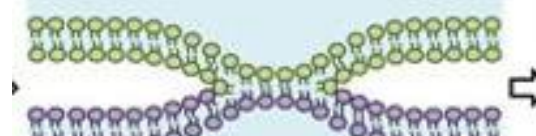

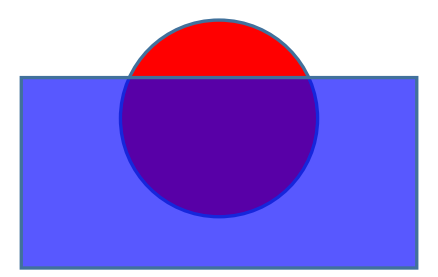

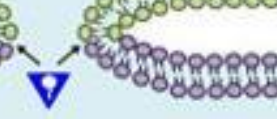




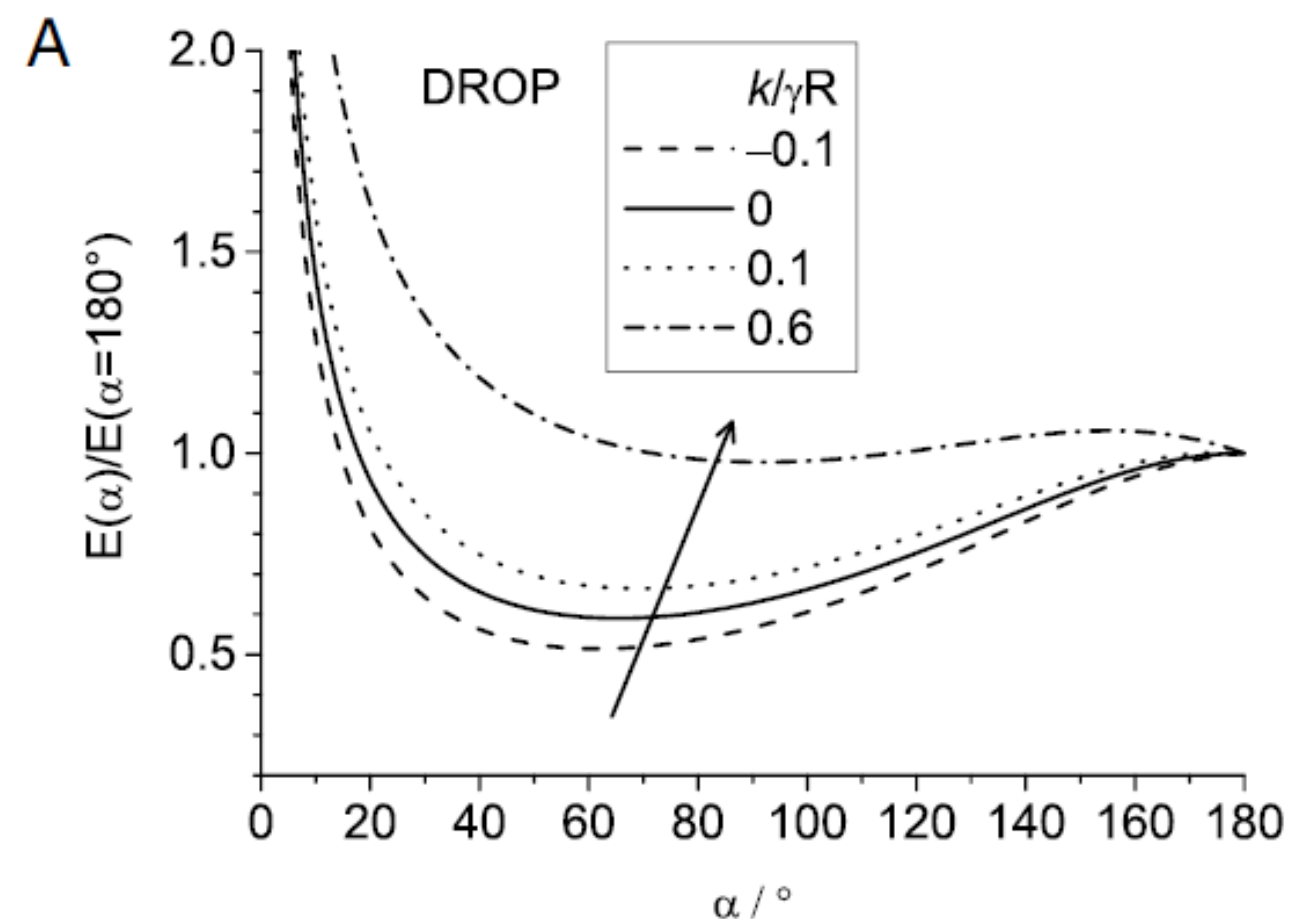

- Minimum could vanish

- Metastable states

- Particles behaves differently depending upon the original location
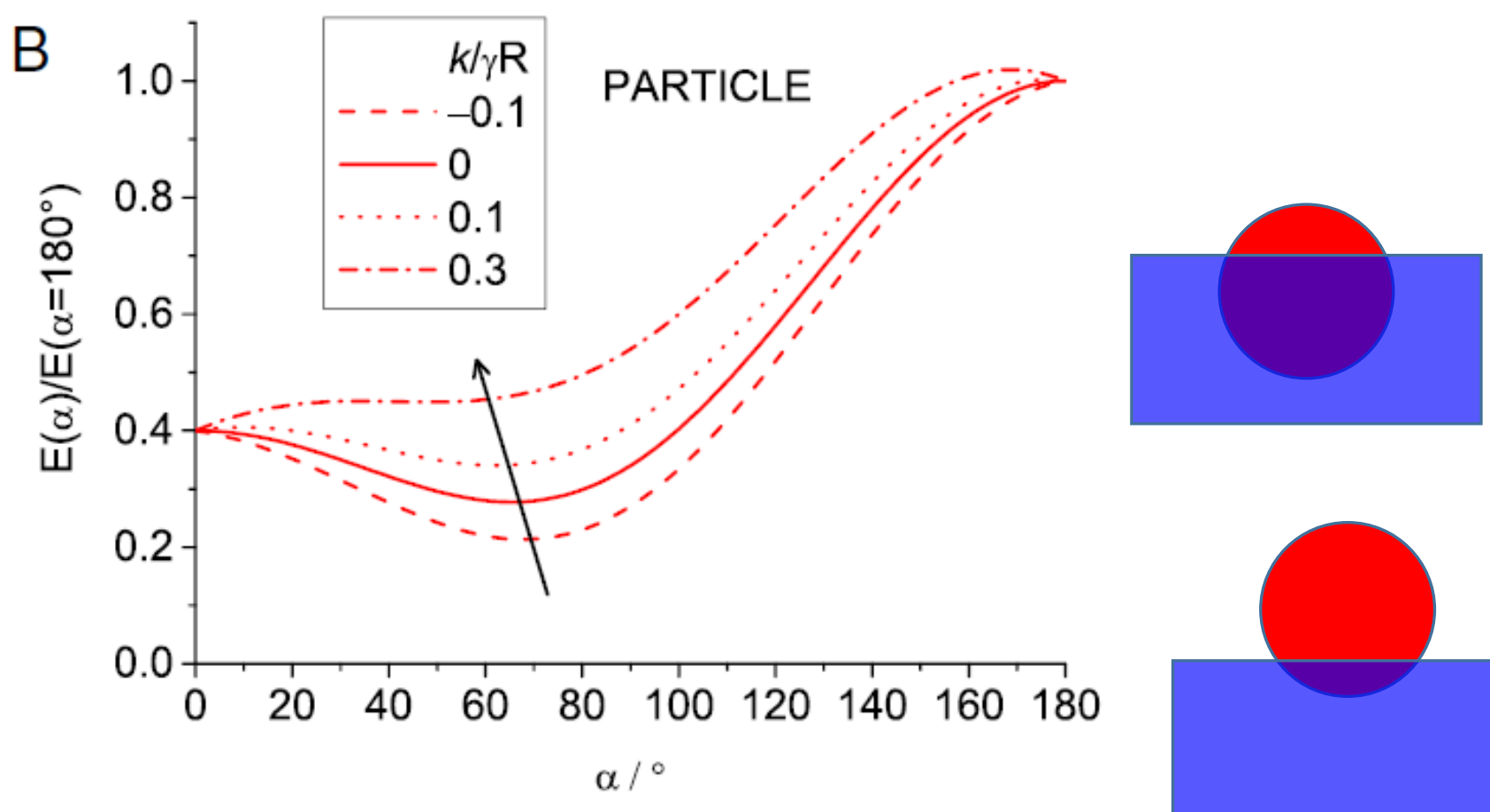

Important for:

nanodrops and nanobubbles

high aspect ratio and nano- particles

And for lipid domains in membranes

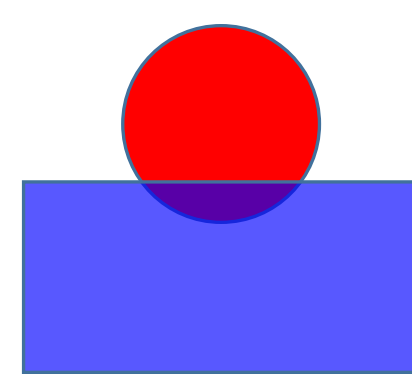




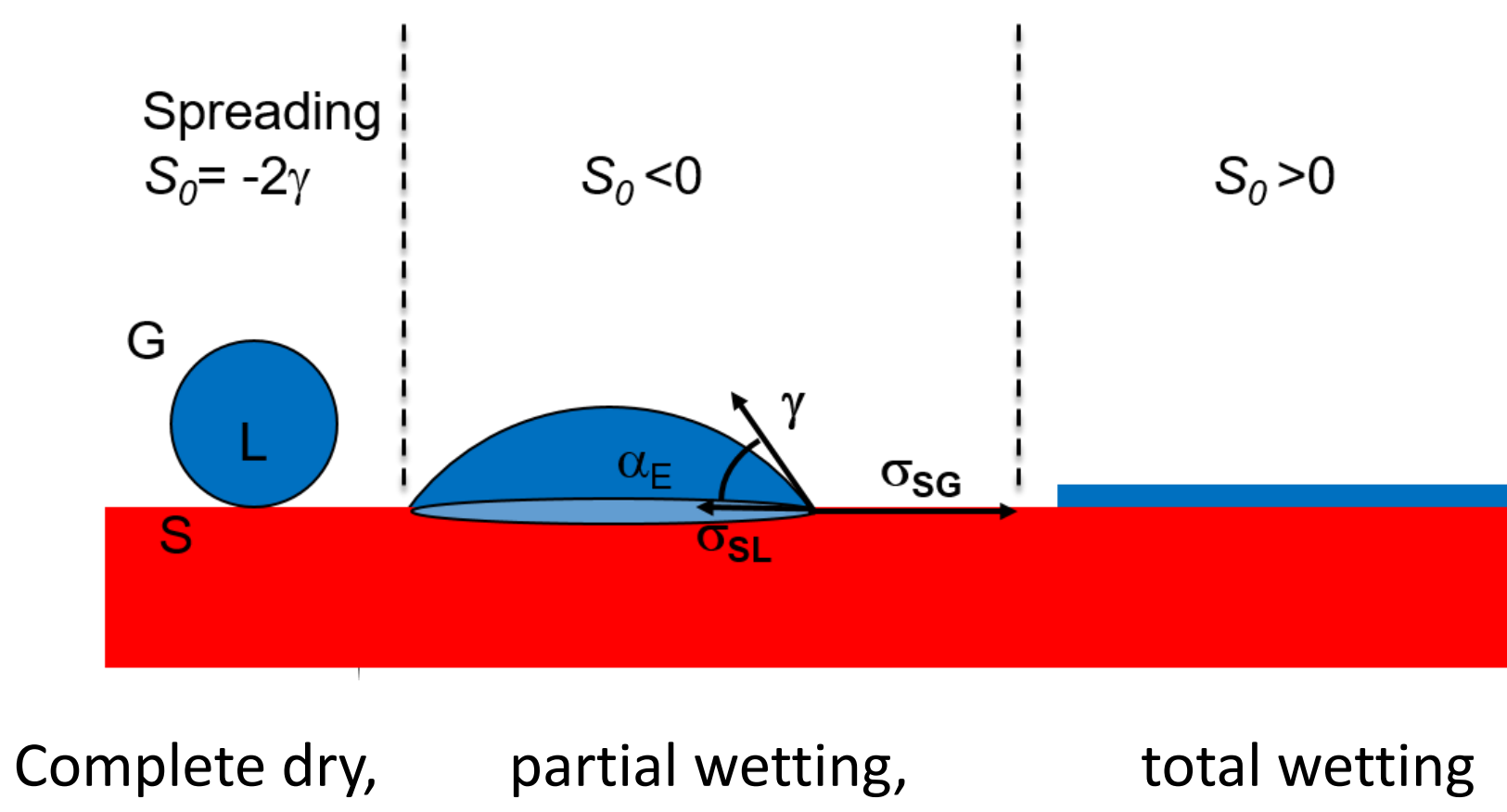

How the wetting state is related to VdW, EDL, disjoining pressure ? 
$\begin{array}{ll} & E(h \rightarrow \infty)=\sigma_{S L}+\gamma \\ \text { Per unit area } & E(0)=\sigma_{S G}\end{array}$

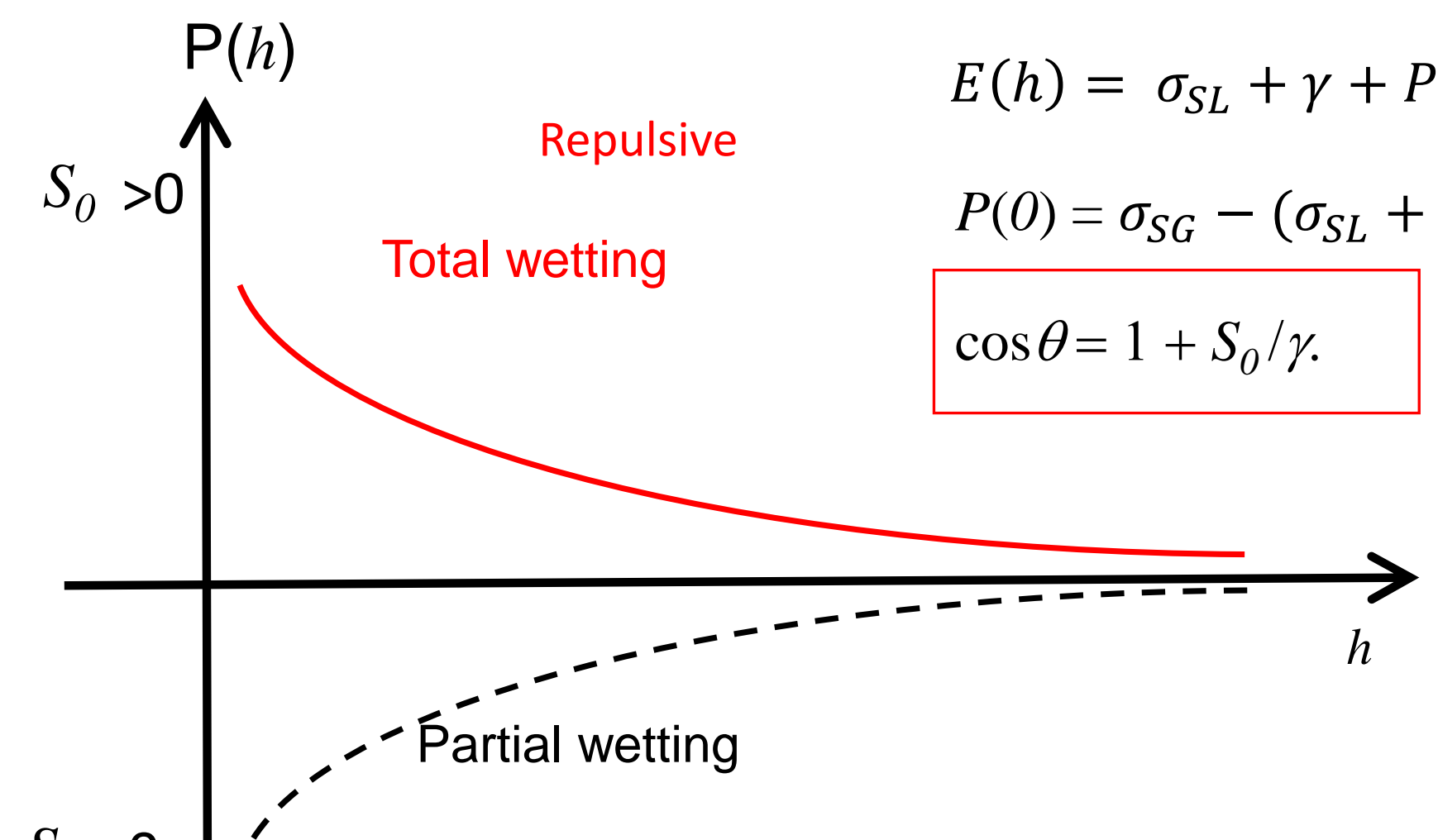

G

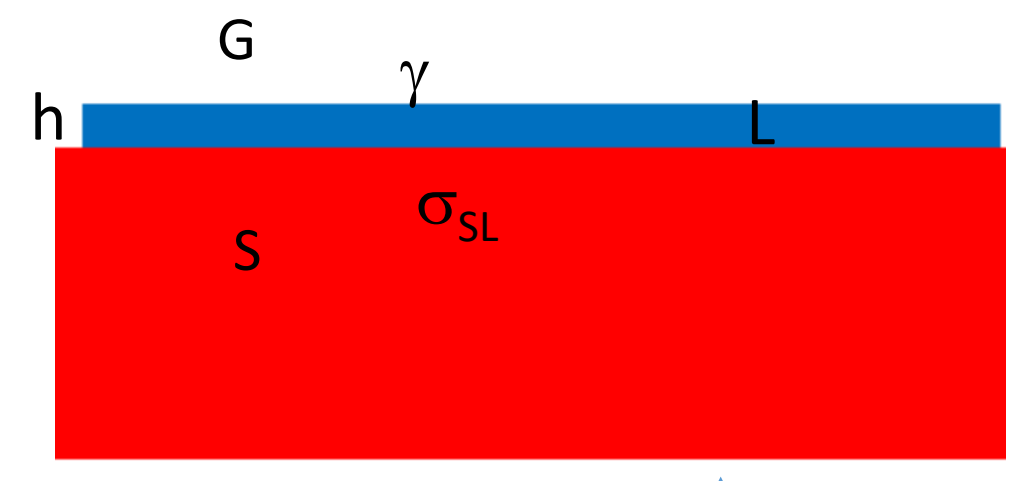

Repulsive

$S_{0}>0$
Disjoining pressure $\Pi(h)=-\frac{d P}{d h}$
If VdW only

Attractive

$$
S_{0}<0
$$

$A_{H}<0$ repulsive

$\mathrm{A}_{\mathrm{H}}>0$ attractive 


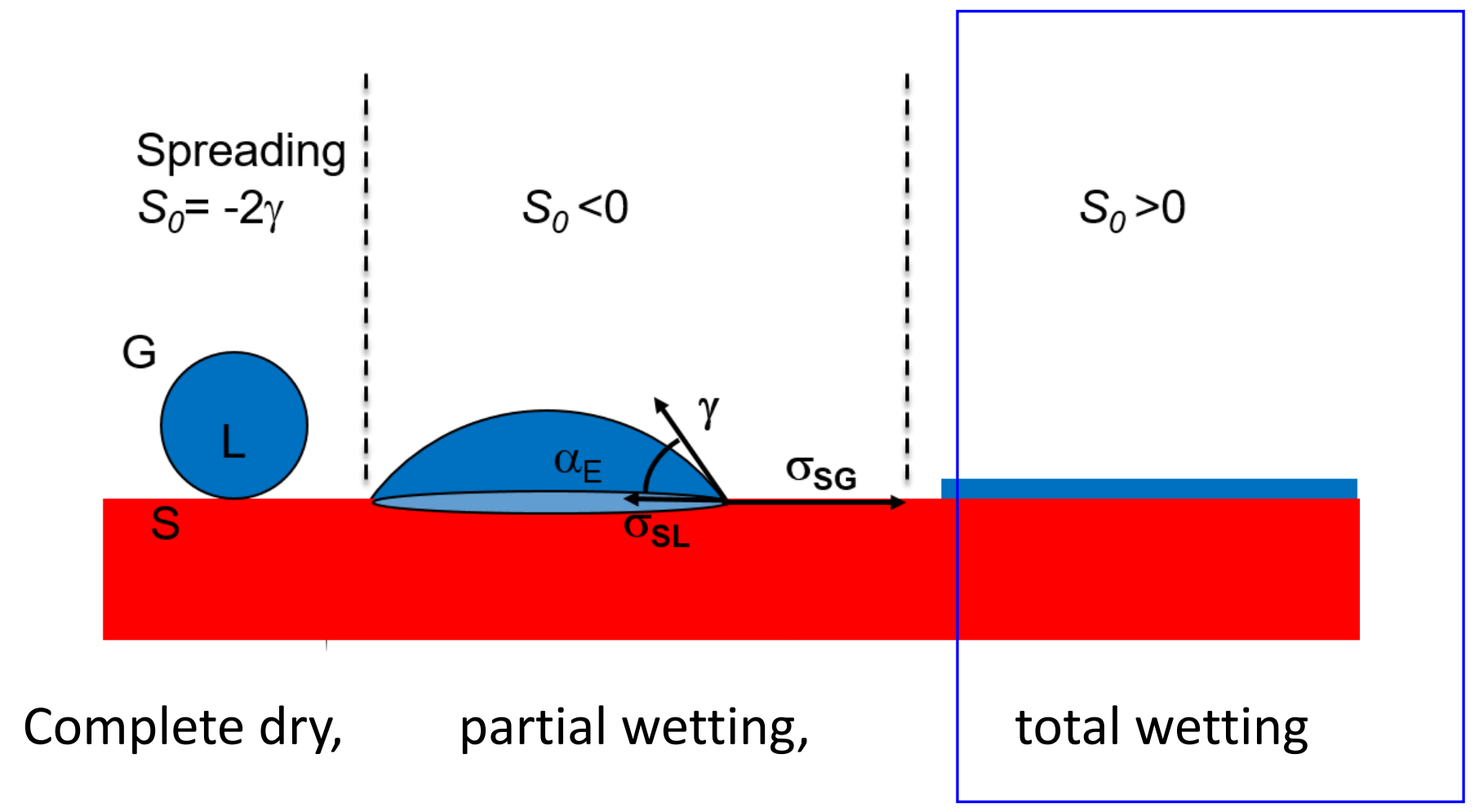

How the wetting state is related to VdW, EDL, disjoining pressure? 


$$
\begin{aligned}
& E(h)=A\left(\sigma_{S L}+\gamma+P(h)\right) \quad \text { Total energy, } \mathrm{V}=\mathrm{const} \\
& \gamma_{\text {film }}=d E(h) / d A=\sigma_{S L}+\gamma+\frac{P(h)+h \Pi}{\text { chemical potential }}
\end{aligned}
$$

$$
\begin{aligned}
& \Pi(h)=D L V O+\text { short range forces } \\
& =\text { Attractition }+ \text { Repulsion }+\cdots
\end{aligned}
$$

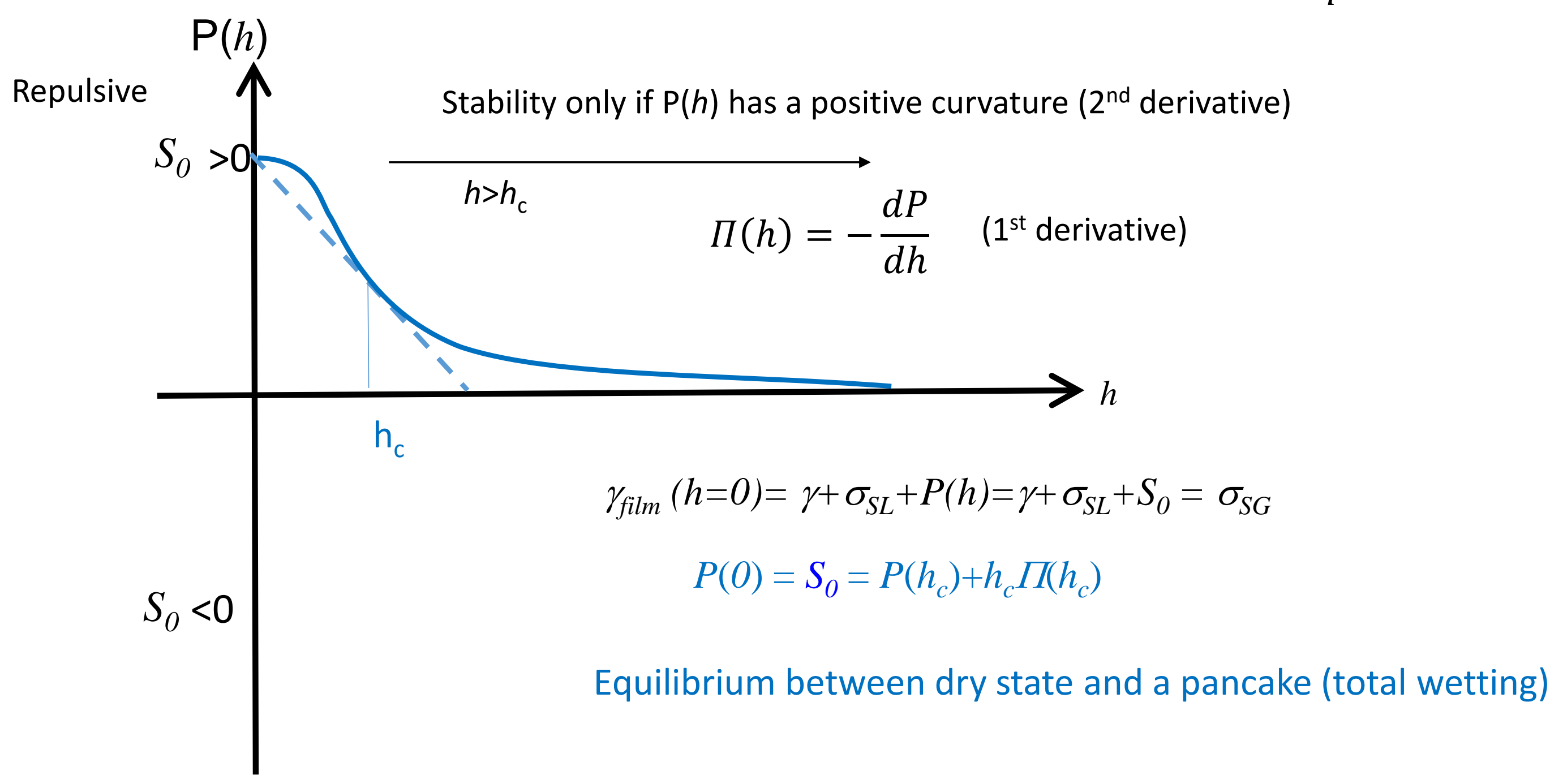




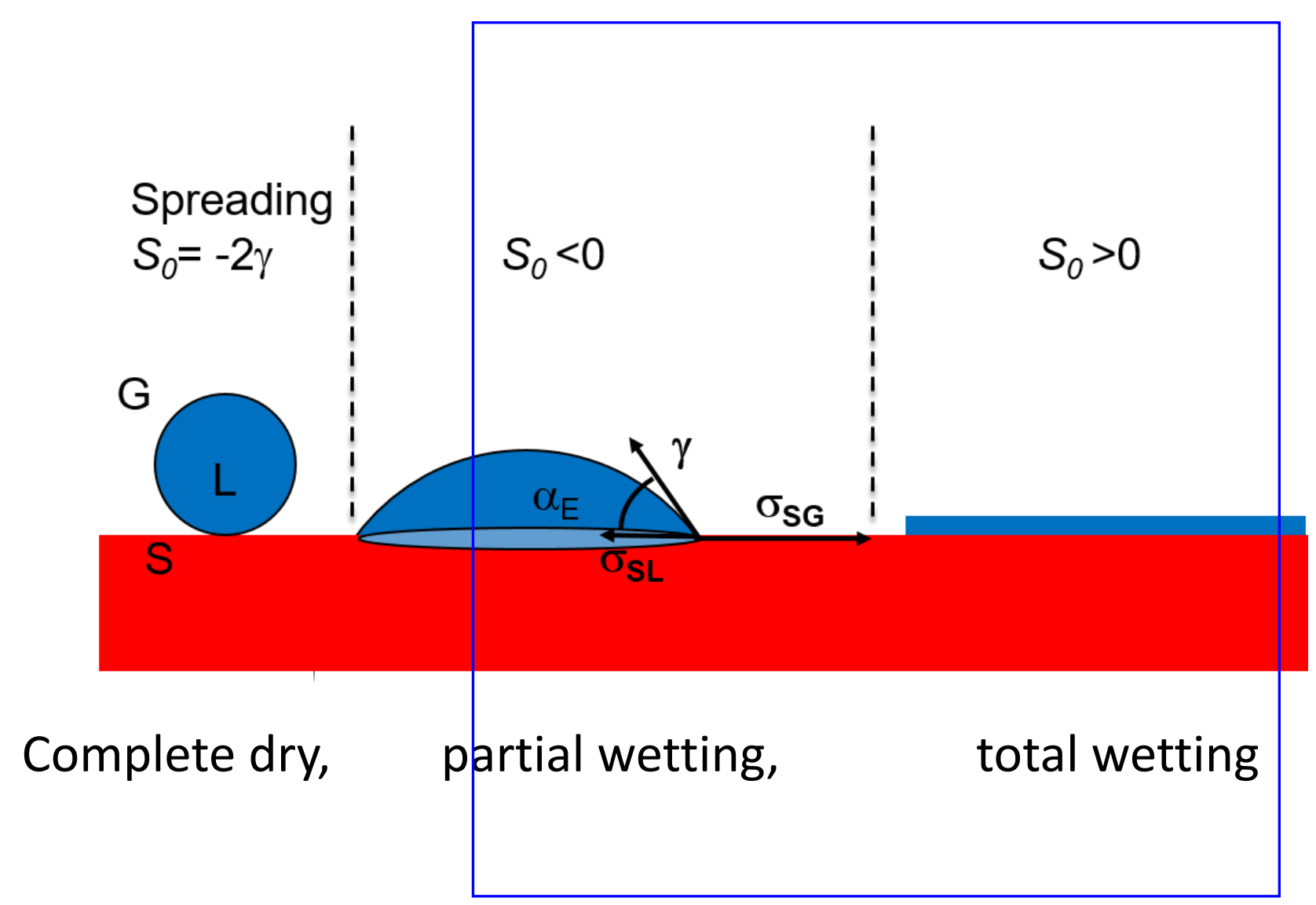

How the wetting state is related to $\mathrm{VdW}, \mathrm{EDL}$, disjoining pressure ? 


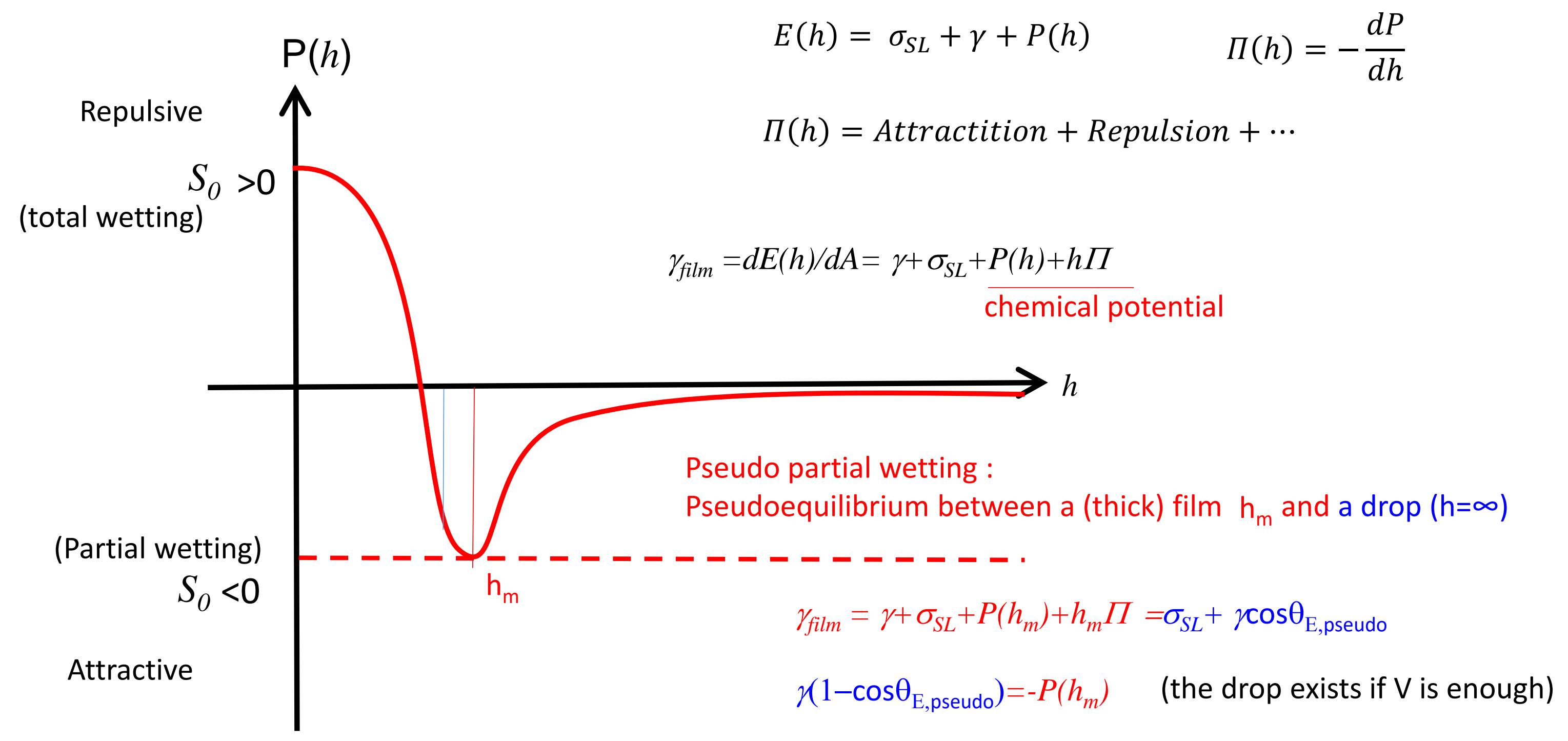

Partial wetting : $\quad\left(1-\cos \theta_{\mathrm{E}}\right)=-S>0$

Interfacial tension and contact angle of the droplet give the potential $P$ at $h_{m}$ ! 


\section{Questions:}

Does the equilibrium contact angle change with the drop (bubble) size?

$$
S_{0}<0
$$

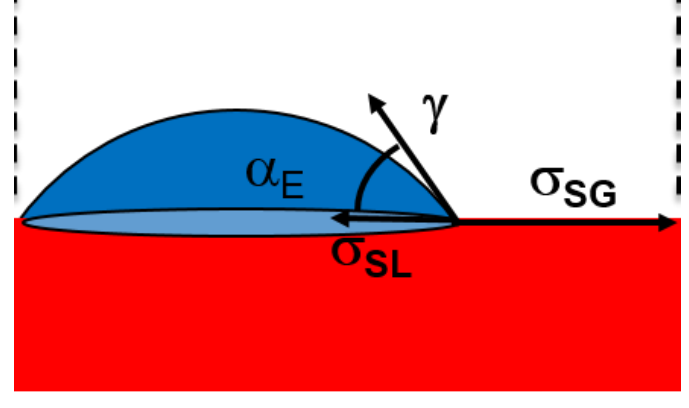

Does the equilibrium contact angle change with the particle size?

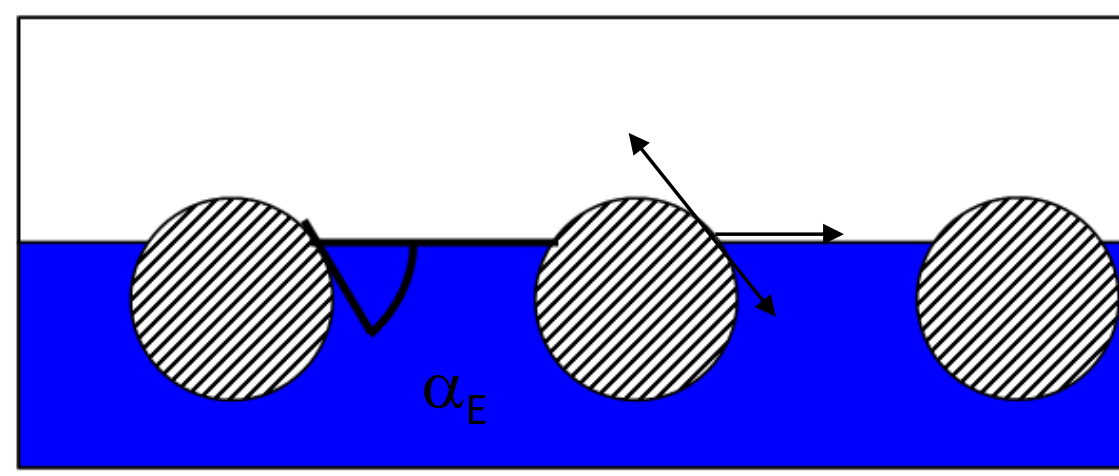


7. Line tension $k$ (important for bilayers, membranes...) for macroscopic drops

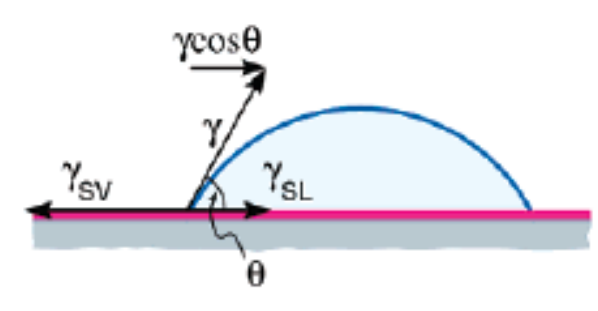

$\cos \alpha_{e q}=\left(\sigma_{S G}-\sigma_{S L}\right) / \gamma$

$$
E(\alpha)=\sigma_{S G} A-\sigma_{S G} A_{0}+\sigma_{S L} A_{0}+\gamma A_{L G}+k 2 \pi R_{0}(\alpha) \sin \alpha .
$$

$\cos \mathcal{A}_{e q}=\cos \alpha_{e q}-\frac{k}{\gamma R_{0}(\alpha)} \frac{1}{\sin \mathcal{A}_{e q}} \quad$ (drop)

$$
E(\alpha)=\gamma A+2 \pi R^{2}\left[\sigma_{S G}(1-\cos \alpha)+\sigma_{S L}(1+\cos \alpha)-\frac{1}{2} \gamma \sin ^{2} \alpha+\frac{k}{R} \sin \alpha\right]
$$

$\cos \mathcal{A}_{e q}=\cos \alpha_{e q}+\frac{k}{\gamma R} \frac{\cos \mathcal{A}_{e q}}{\sin \mathcal{A}_{e q}} \quad$ (particle)

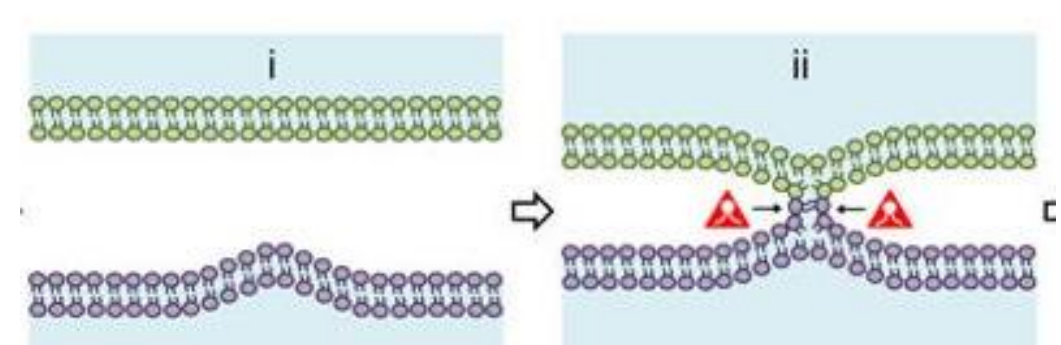

iii

iv

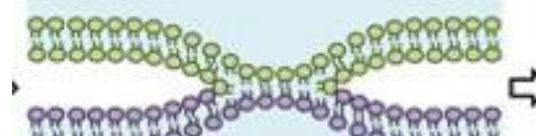

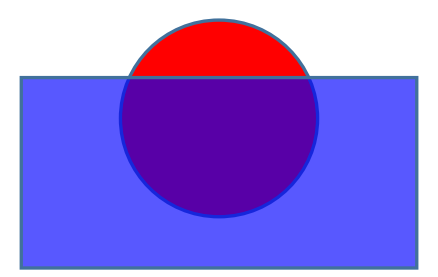

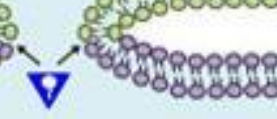


- Size dependent contact angle

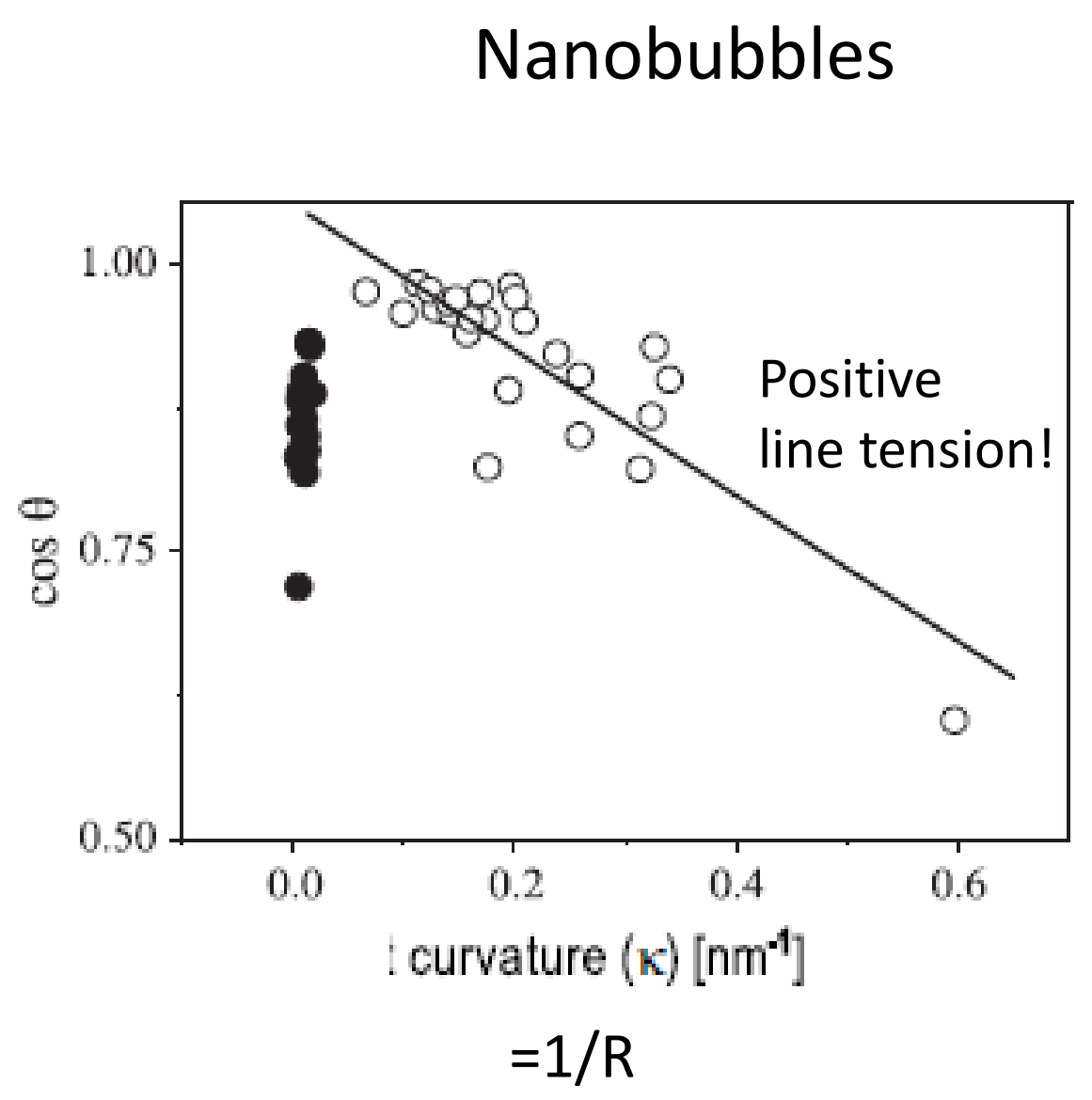

Lohse, Zhang, Rev Mod Phys 2015
Nanodrops
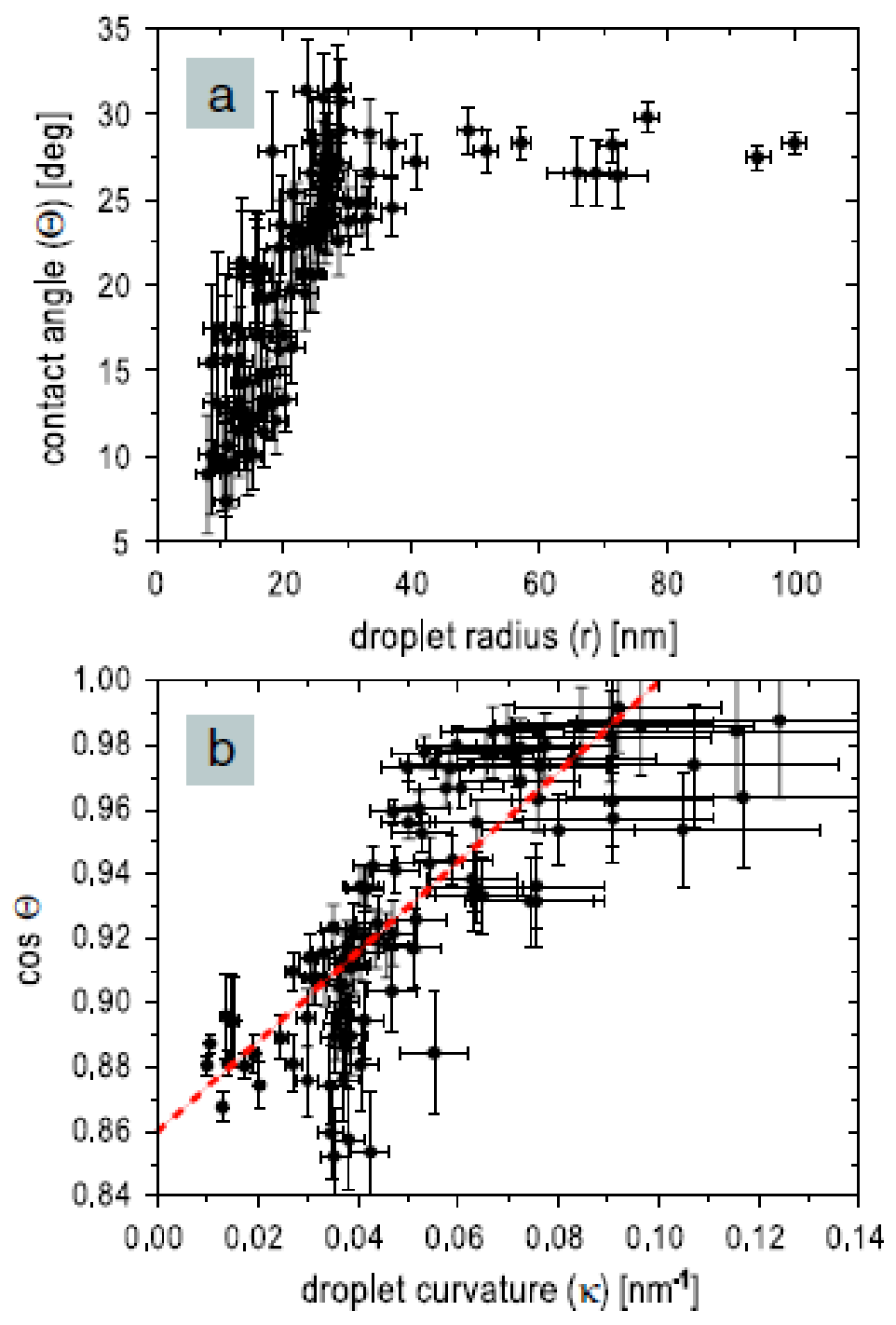

Negative line tension! 
Particles at PS-air interface

PRL 109, 196101 (2012)

PHYSICAL REVIEW

LETTERS

week ending 9 NOVEMBER 2012

Influence of Line Tension on Spherical Colloidal Particles at Liquid-Vapor Interfaces

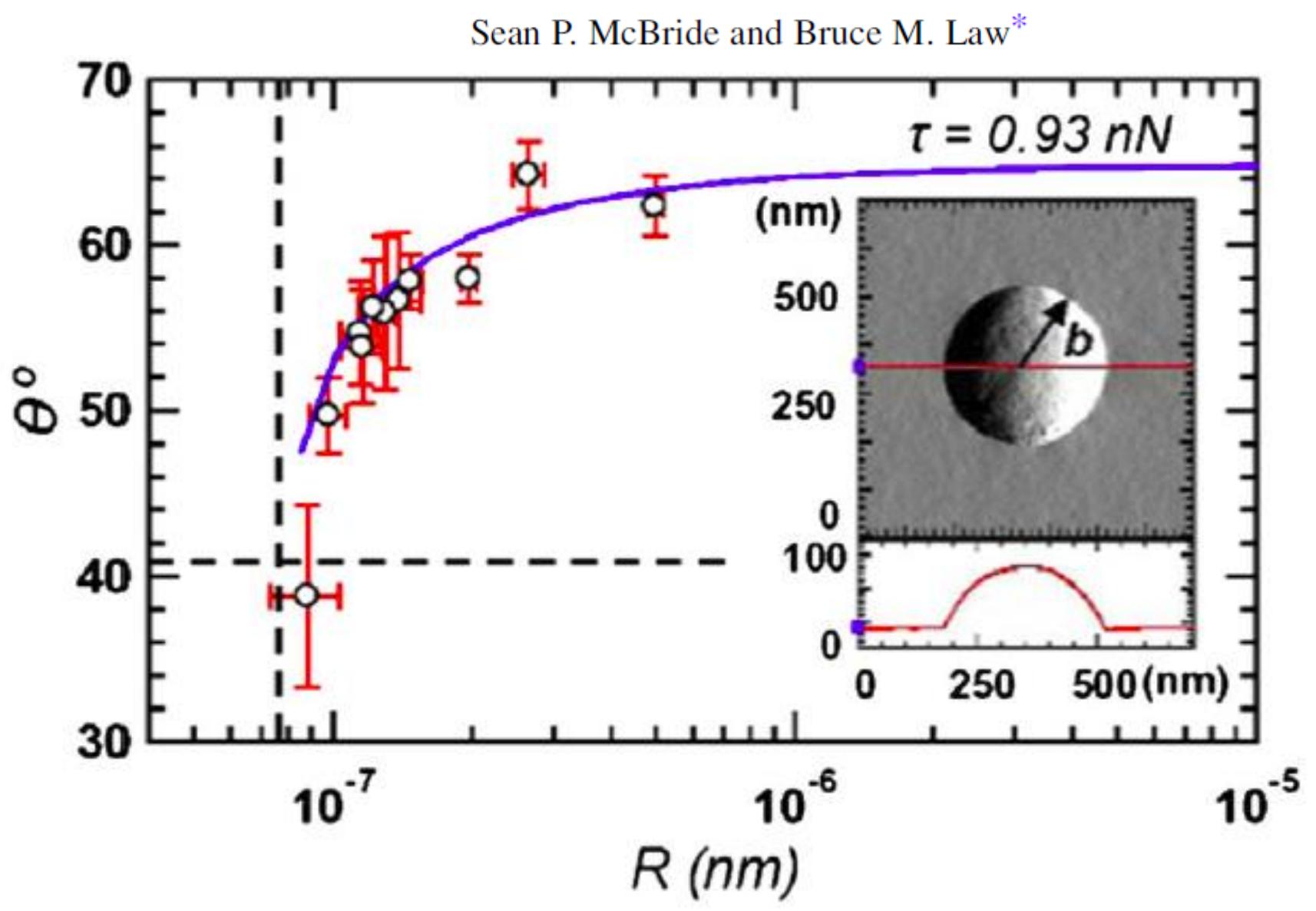




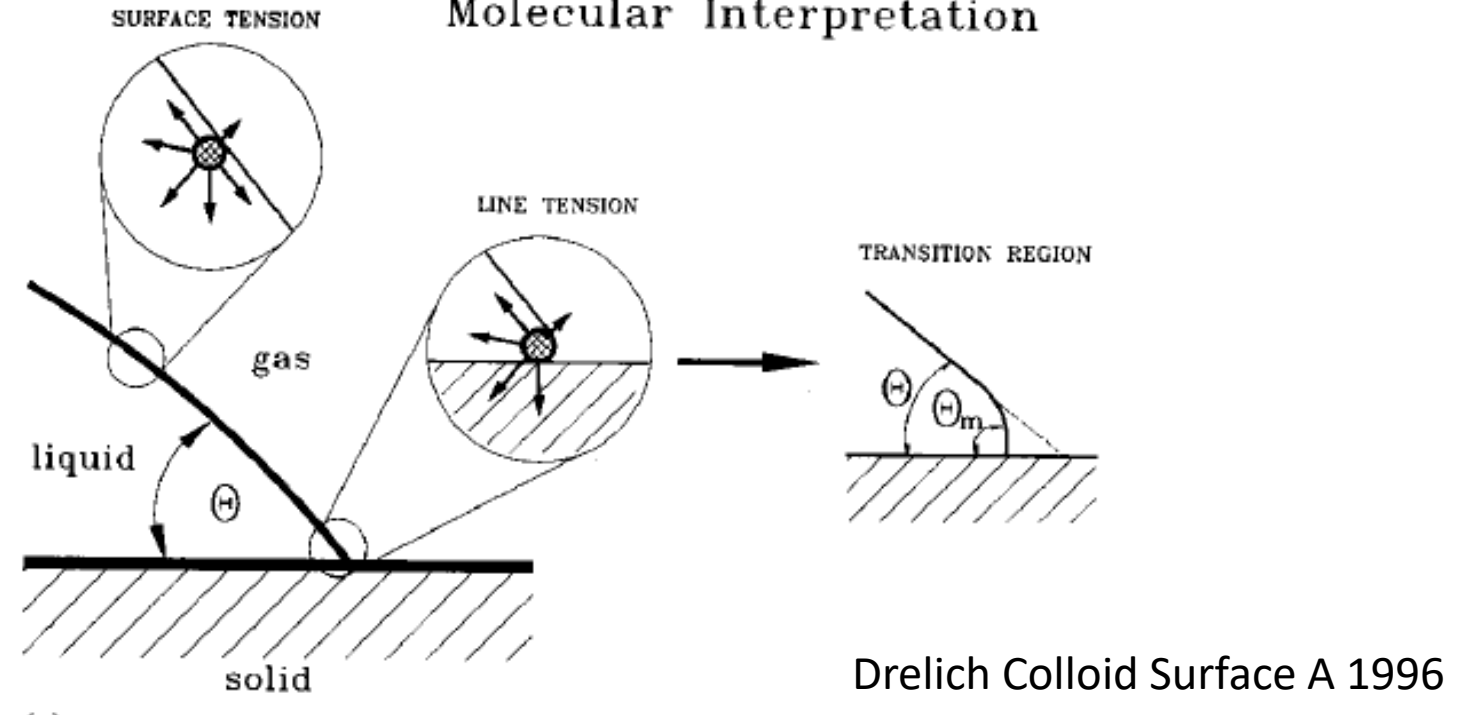

(a)

LINE TENSION

Microscopic Interpretation

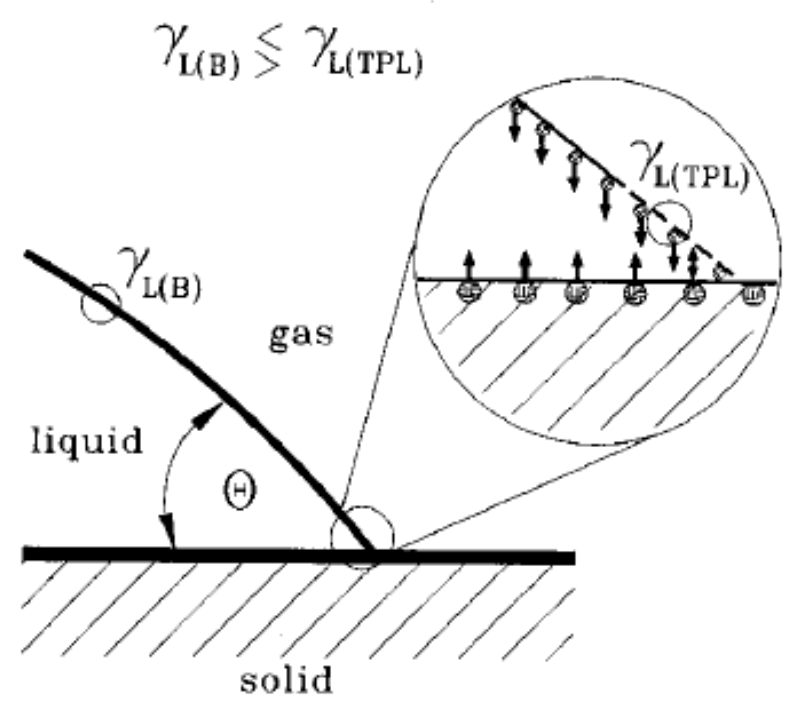

TRANSITION REGION

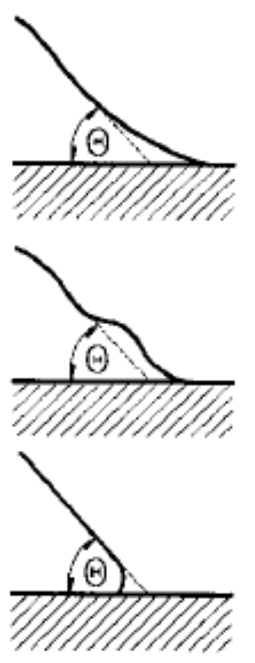

As we did for the interfacial tension, we can estimate line tension values as nearest neighbours exchanges

Energy cost/unit length

$\mathrm{kT} / \mathrm{R}=4 \times 10^{-21} /\left(0.3 \times 10^{-9}\right)=10^{-11} \mathrm{~N}$

(b) 
Microscopic interpretation: line tension reflects the disjoining pressure
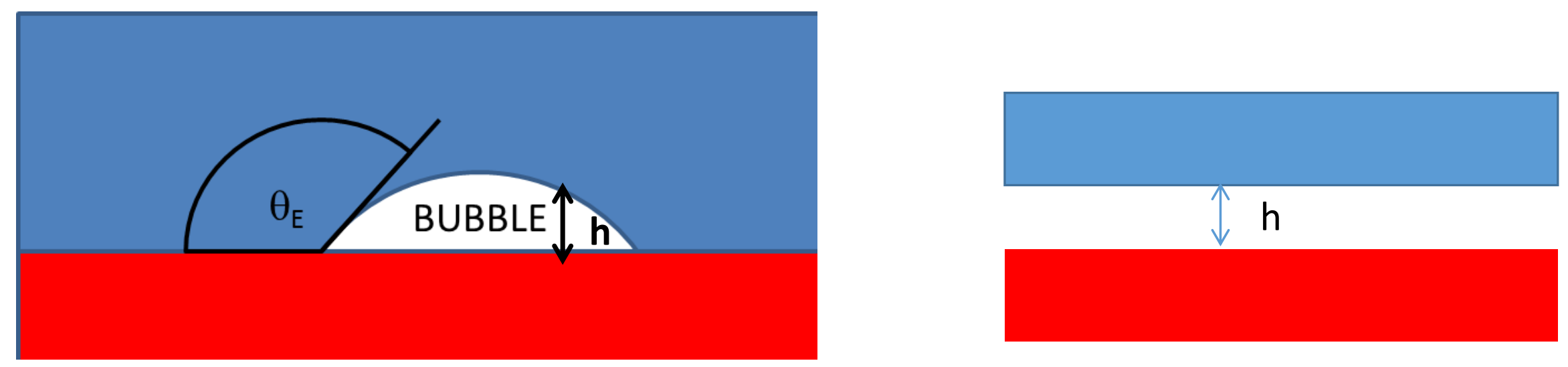

$$
\begin{array}{r}
\gamma \Sigma+\Pi(h)+\rho g h=\mathrm{P}_{0} \\
\text { curvature }\left(=1 / \mathrm{R}_{0}\right)
\end{array}
$$

If $\mathrm{R}_{0}>$ ca $1 \mu \mathrm{m}$, capillarity dominates $\gamma \Sigma>\Pi(h)$

If $\mathrm{R}_{0}<$ ca $1 \mu \mathrm{m}$, surface forces dominates $\gamma \Sigma<\Pi(h)$

Nanobubbles

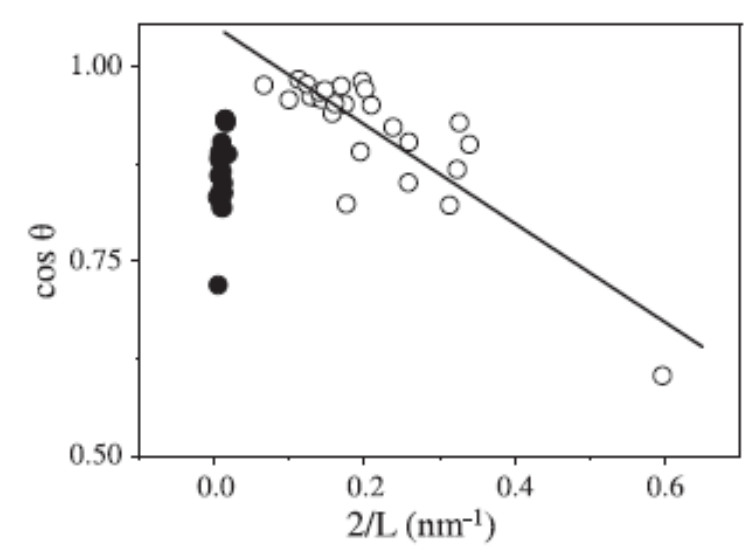




\section{EXPERIMENTS show spherical cap profiles for nanodrops and nanobubbles}
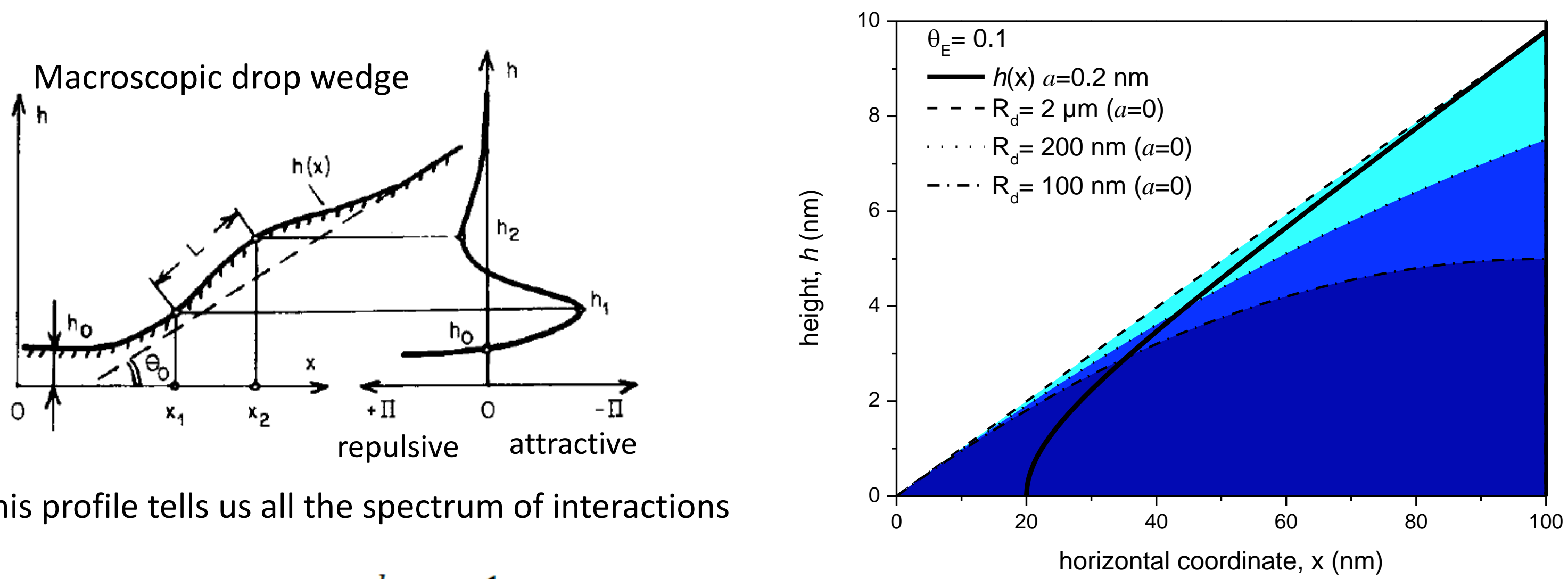

$\cos \mathcal{A}_{e q}=\cos \alpha_{e q}-\frac{k}{\gamma R_{0}(\alpha)} \frac{1}{\sin \mathcal{A}_{e q}}$

Line tension can not be a constant for nanodrops ! It must be length scale dependent! 


\section{Attractive VdW}

A

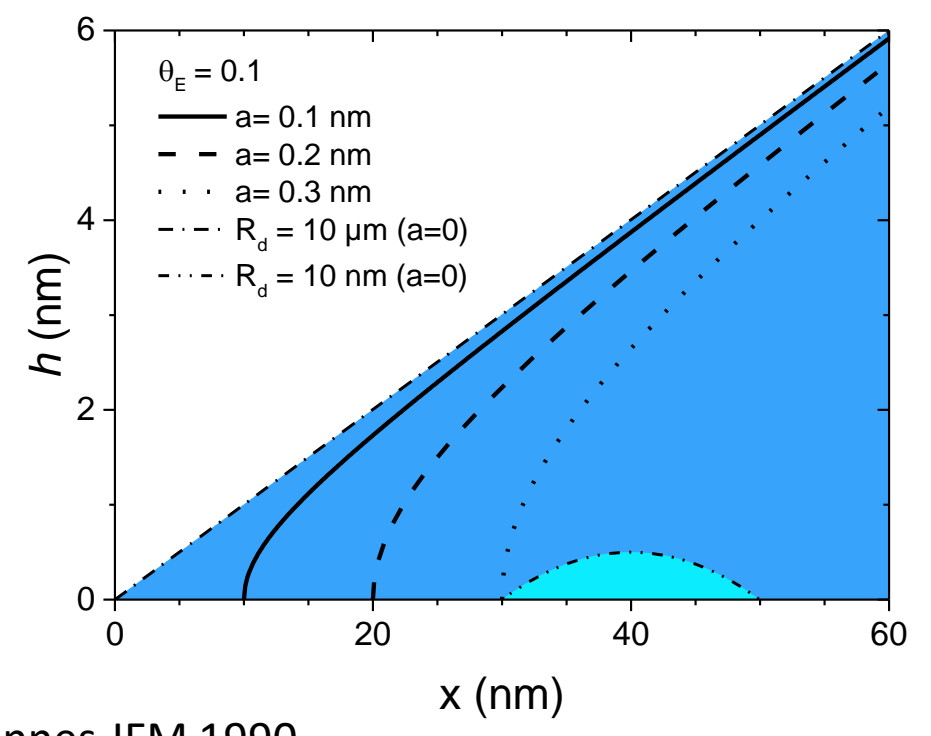

De Gennes JFM 1990

Local contact angle increases if $h$ decreases

$=$

Positive line tension

\section{Repulsive VdW}

B

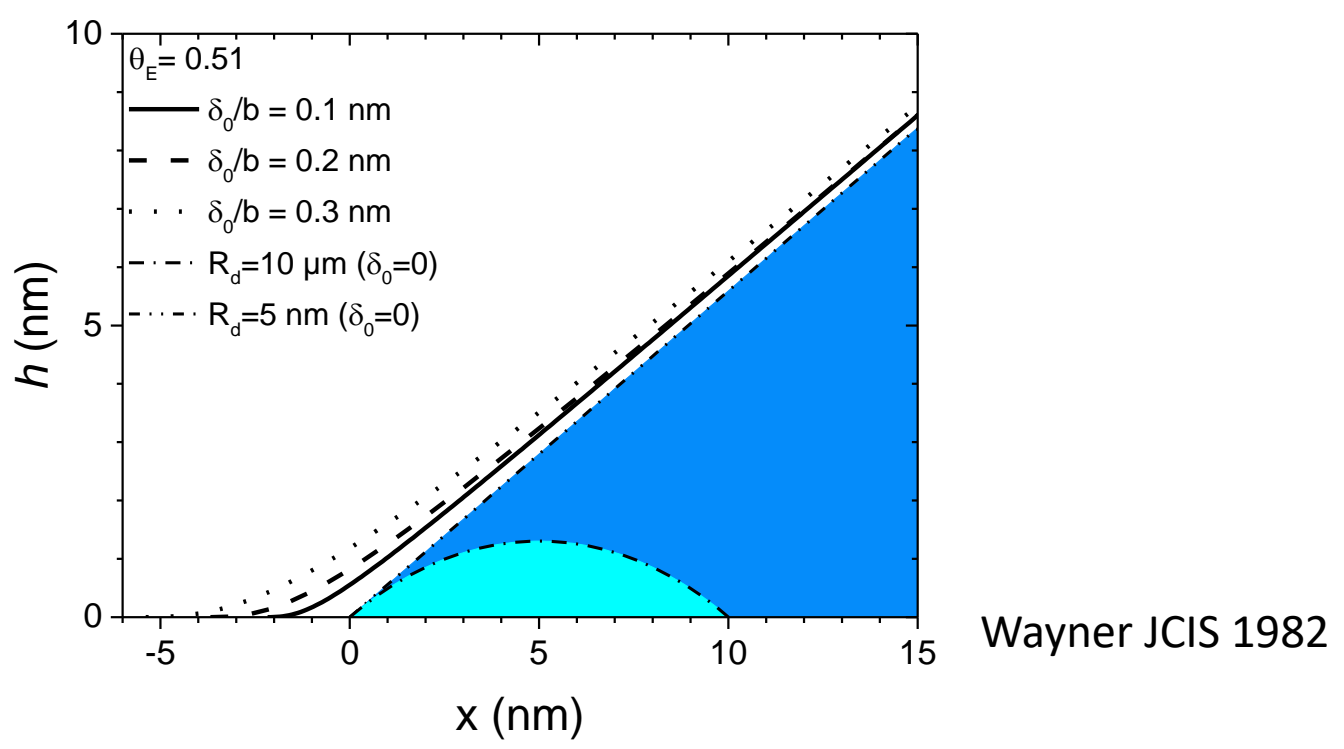

the meniscus is at equilibrium with a pancake film

Local contact angle decreases if $h$ decreases

$=$

Negative line tension

Criterion The contact angle of a nanodrop = local contact angle 


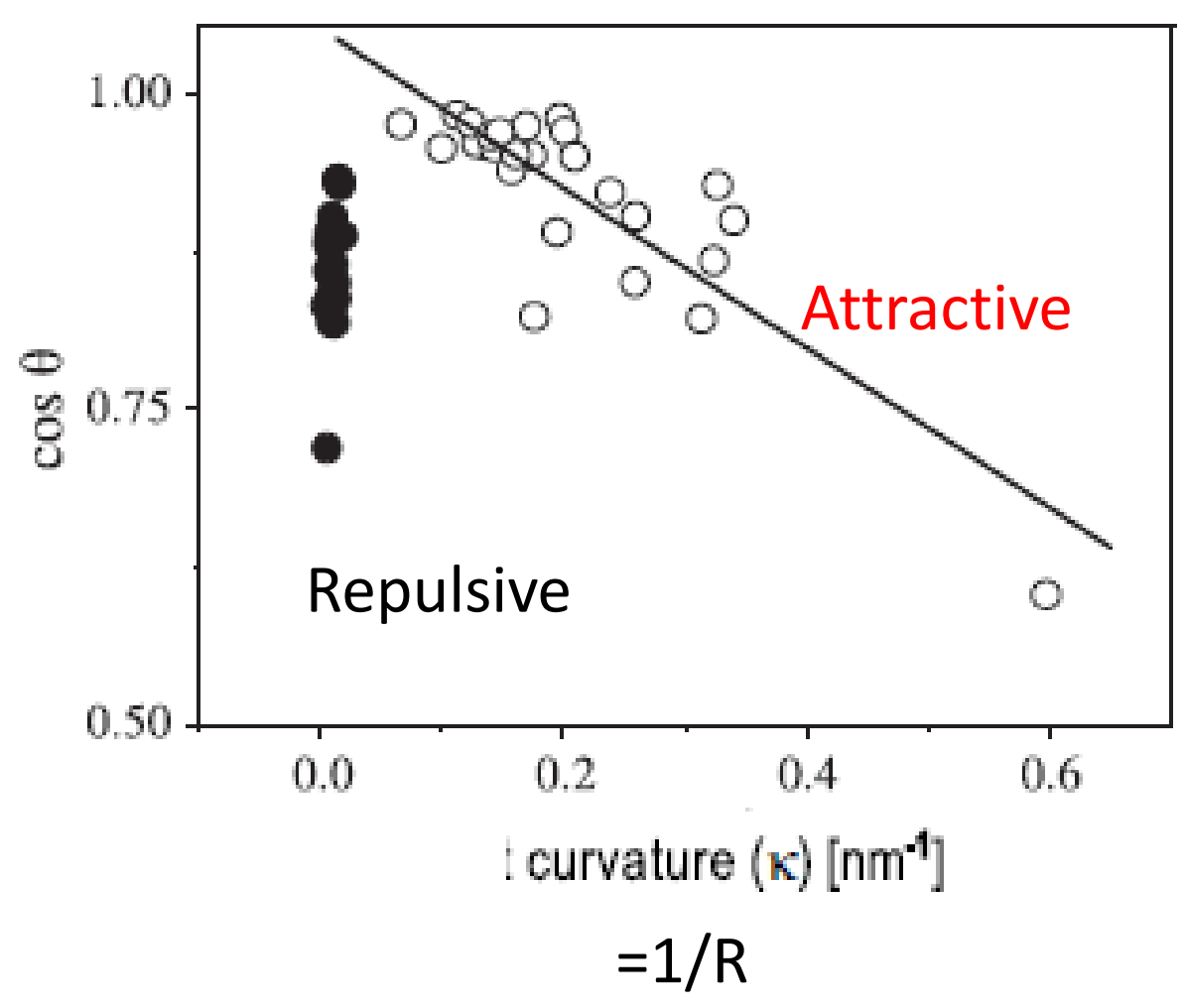

This profile tells us all the spectrum of interactions between the two interface (disjoining pressure) 


\section{First Summary Part 2: Wetting}

Wetting states

- Contact angle

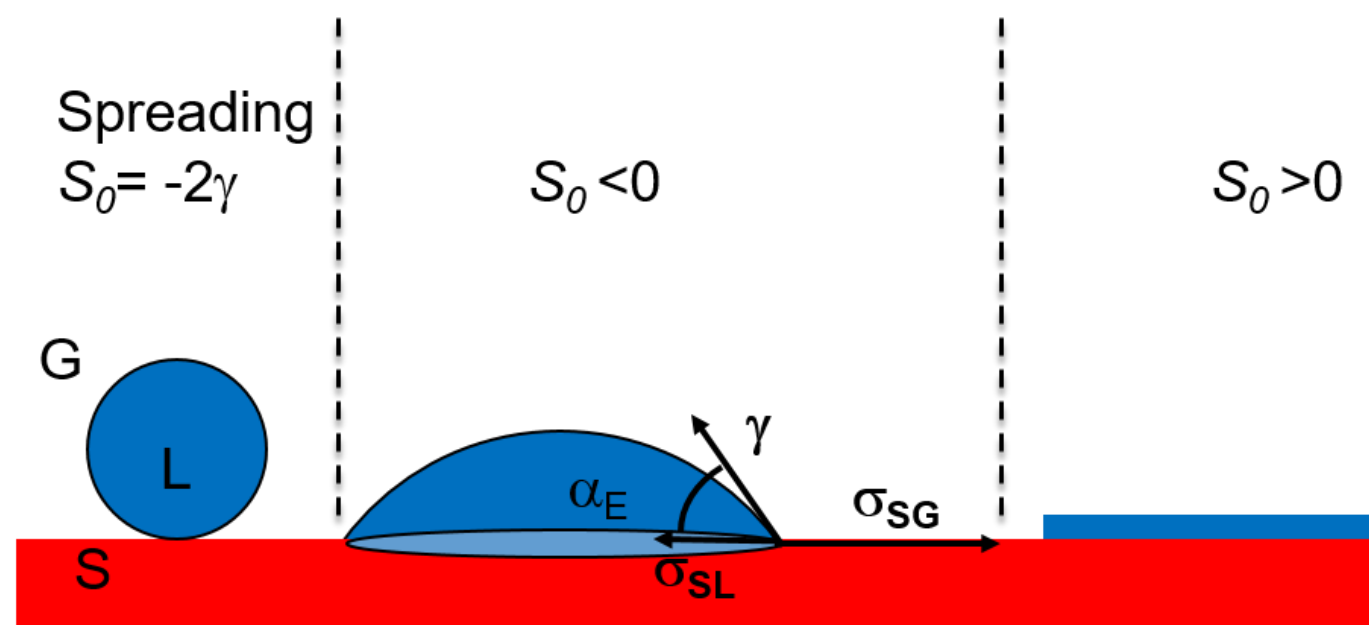

- Line tension

- Role of Disjoining Pressure (VdW, EDL, DLVO + ....)

- Contact angle depends on the system size

- line tension is connected to the disjoining pressure

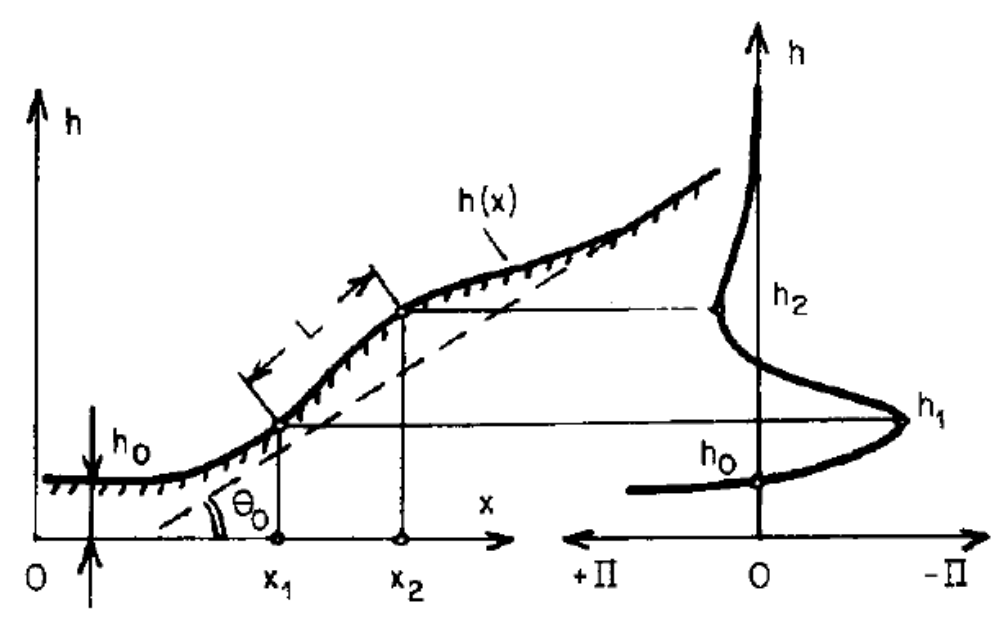


Roughness: Free energy (Wenzel model)

Drop:

$$
\begin{aligned}
& E(\alpha)=\sigma_{S G} A-\sigma_{S G} A_{0}+\sigma_{S L} A_{0}+\gamma A_{L G} . \text { (no roughness) } \\
& E(\alpha)=\sigma_{S G} A-\sigma_{S G} A_{s}+\sigma_{S L} A_{s}+\gamma A_{L G} \\
& \cos \alpha_{W}=r \cos \alpha, \text { where } r=A_{s} / A_{O} \quad G
\end{aligned}
$$

Particle:
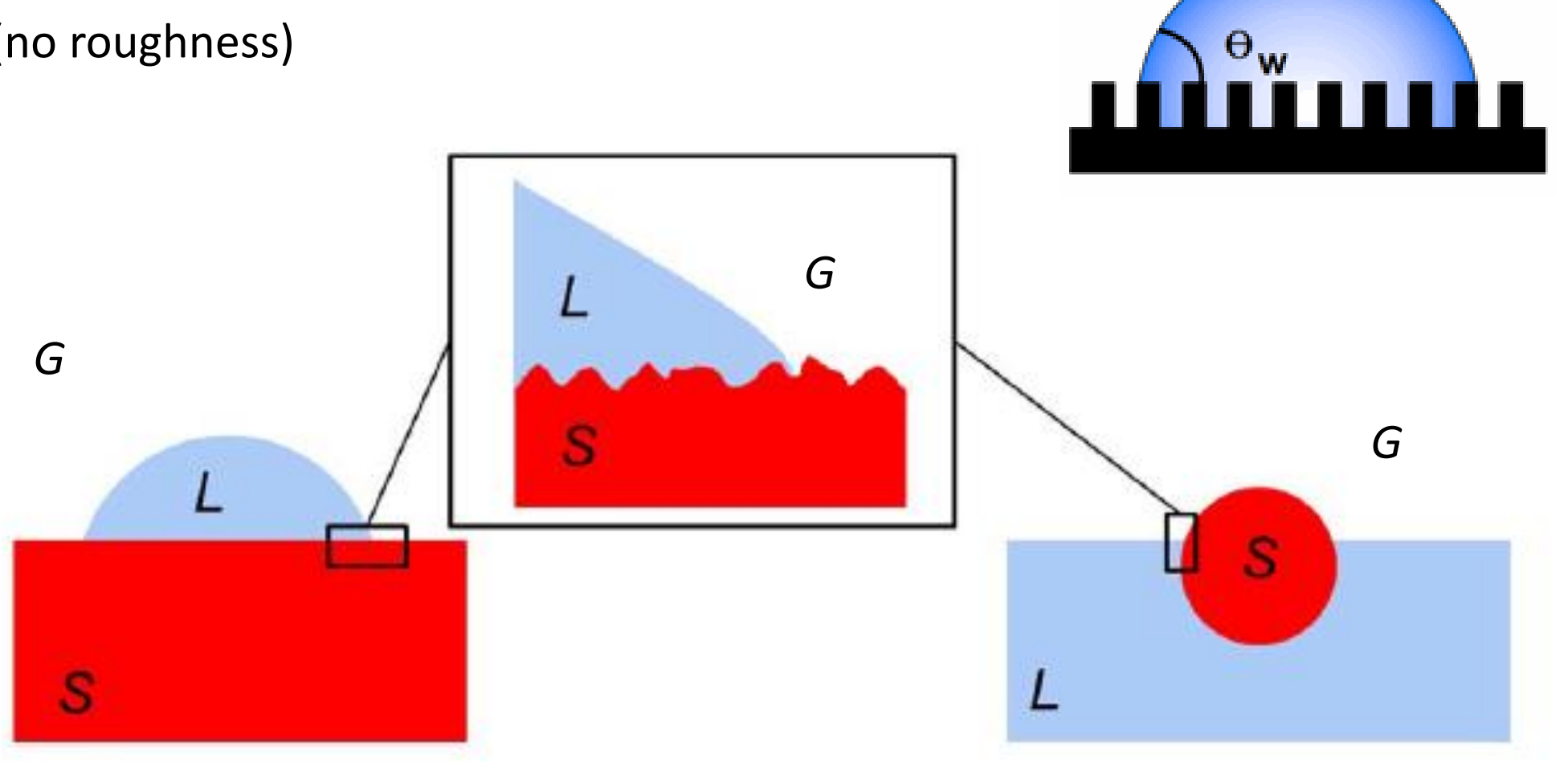

$$
E(\alpha)=\gamma \mathrm{A}+2 \pi R^{2}\left[r \gamma_{S L}(1+\cos \alpha)+r \gamma_{S G}(1-\cos \alpha)-\frac{1}{2} \gamma \sin ^{2} \alpha\right]
$$



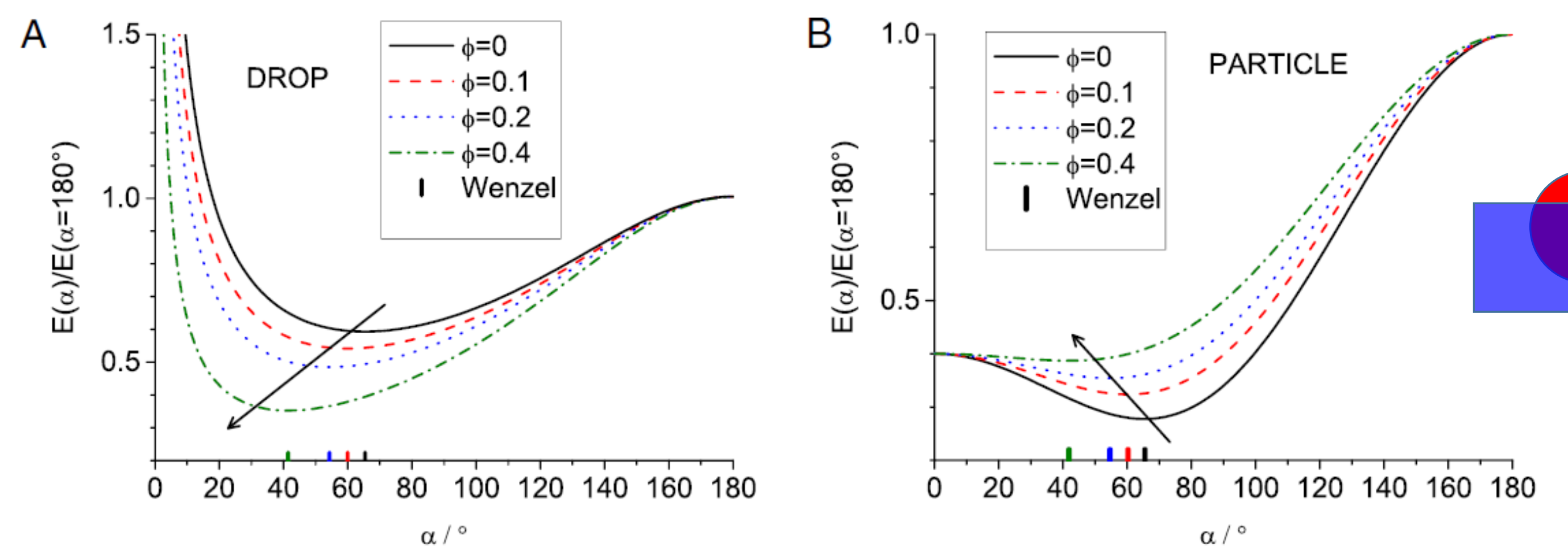

Adhesion strength can be tuned by roughness

Minimum is deeper for drops and could vanish for paricles

Stocco, A. Nobili, M. Advances in Colloid and Interface Science 2017 
Experiments: rough particles

Polymer NPs coating, smoothing by good solvent exposure

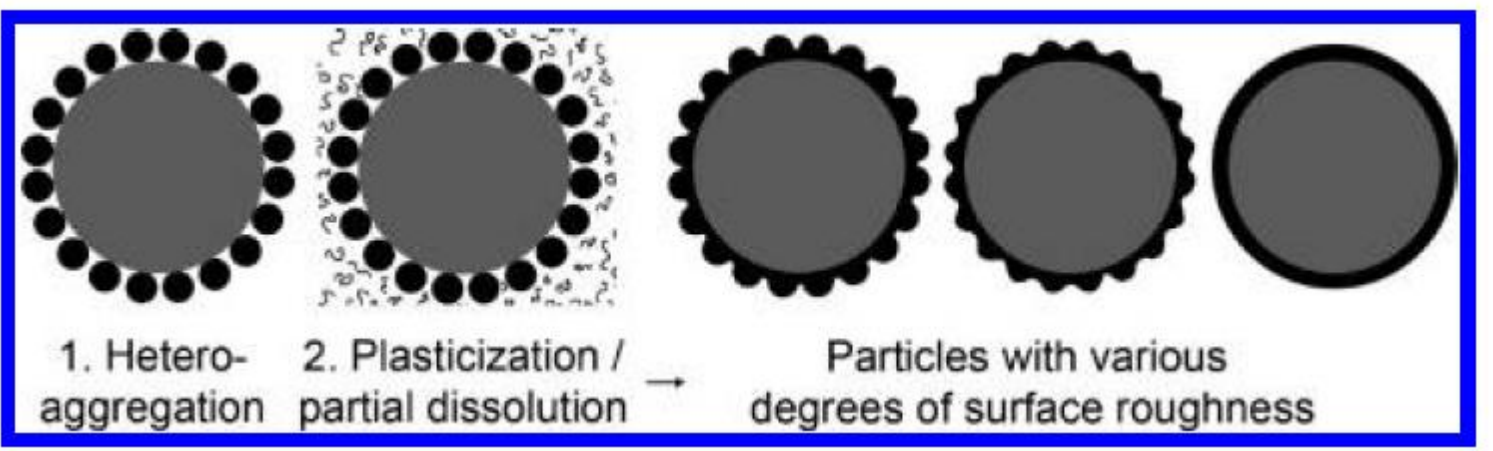

San-Miguel, Behrens Langmuir 2012

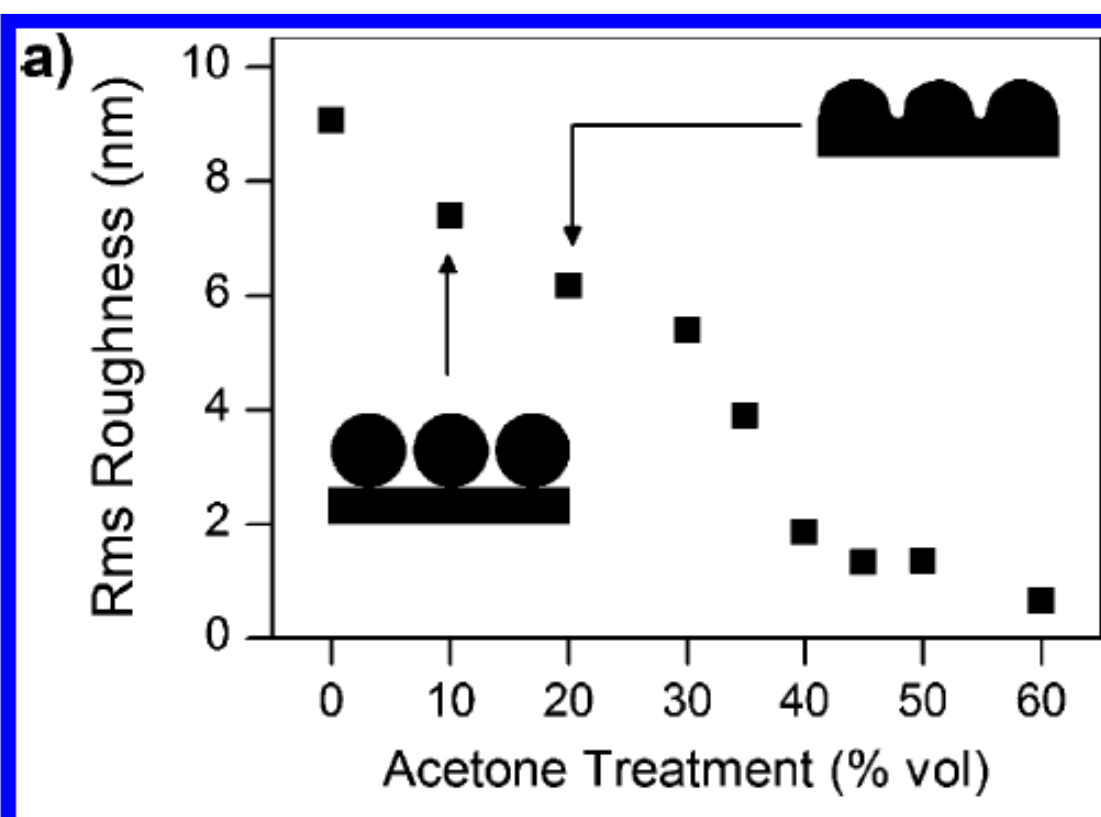

b)

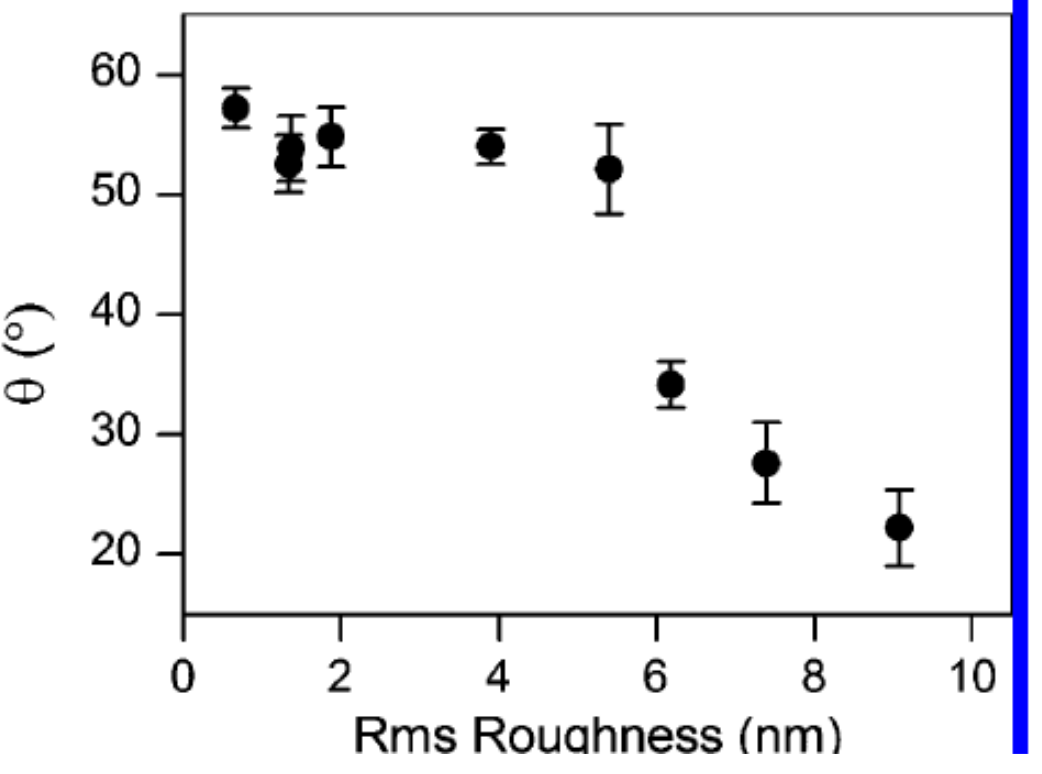


For planar solids with area fractions $\phi$ of materials 1 and $2, \phi_{1}+\phi_{2}=1$ :

$E(\alpha)=\phi_{1}\left(\sigma_{S 1 G} A-\sigma_{S 1 G} A_{0}+\sigma_{S 1 L} A_{0}\right)+\phi_{2}\left(\sigma_{S 2 G} A-\sigma_{S 2 G} A_{0}+\sigma_{S 2 G} A_{0}\right)+\gamma A_{L G}$

At the energy minimum, the "Cassie-Baxter" contact angle is defined as:

$\cos \alpha_{C B}=\phi_{1} \cos \alpha_{E, 1}+\phi_{2} \cos \alpha_{E, 2}$

Air trapped - « fakir » effect

IF 2 is air, $a_{E, 2}=180^{\circ}$

IF $\phi$ is close to zero $\alpha_{C B}$ is close to $180^{\circ}$

$\cos \alpha_{C B}=\phi \cos \alpha_{E, 1}+(1-\phi)-1=$

$\cos \alpha_{C B}=\phi \cos \alpha_{E, 1}-1+\phi$

Where $\phi=$ Post area fraction or hydrophilic area fraction
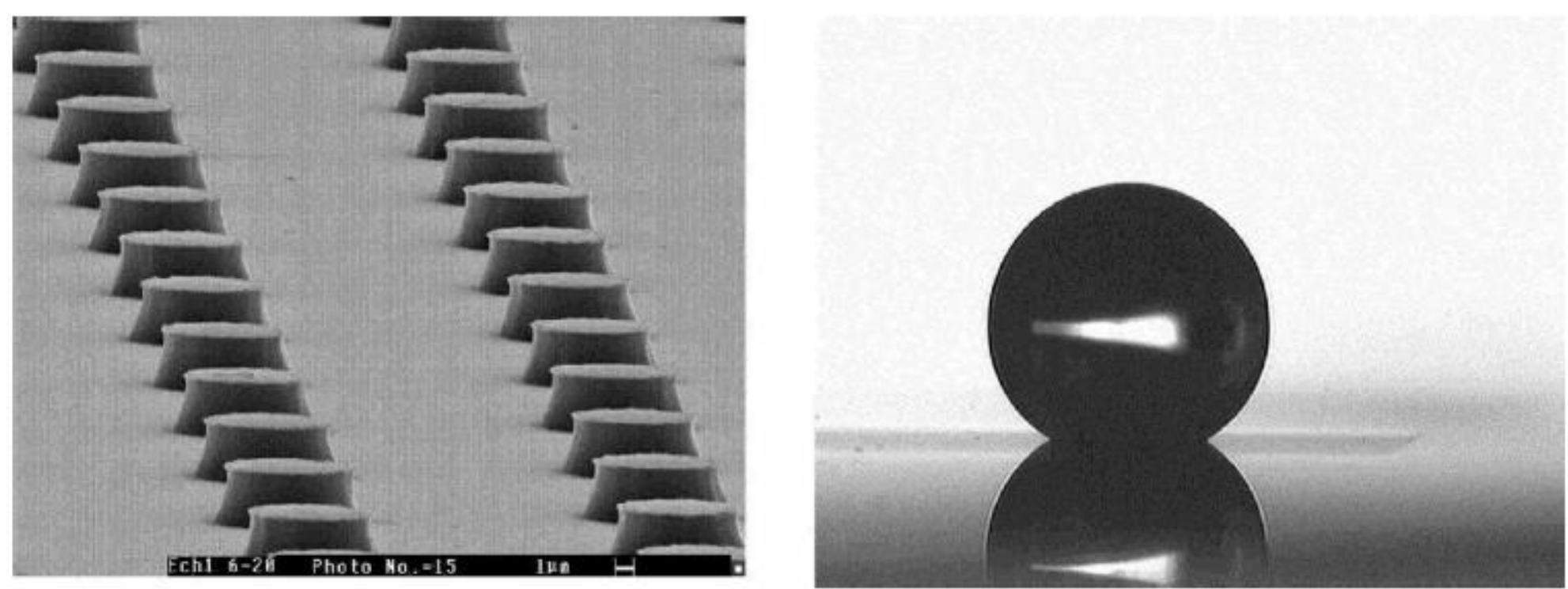
Surfaces are not smooth, they contain defects

$\rightarrow$ Contact angle hysteresis

a)

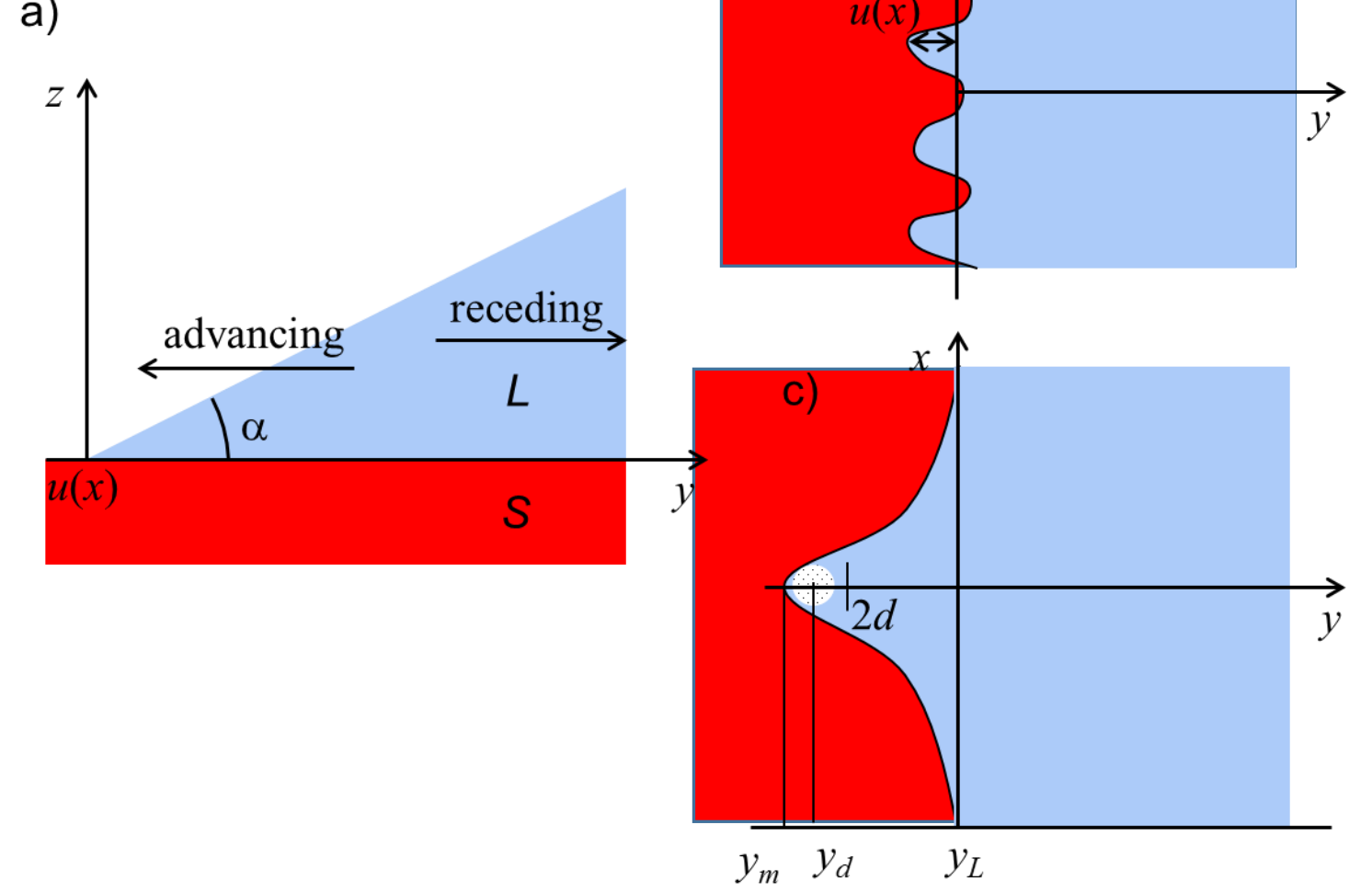

d)

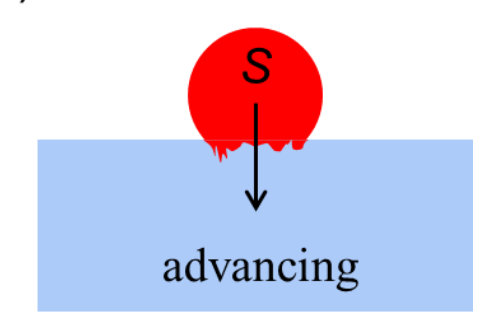

e)

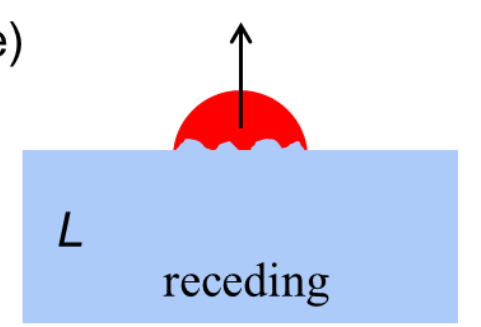

In presence of an isolated defect of size $d$ and force $f_{d}=\gamma d\left[\cos \left(\alpha_{d}\right)-\cos \alpha\right]$, (where $\alpha_{d}$ is the contact angle on the defect) the contact line on the solid takes the form

$u(x)=\frac{f_{d}}{\pi \gamma \sin ^{2} \alpha} \ln \left(\frac{L}{|x|}\right)$,

Where $L$ is a macroscopic distance related to the system size 


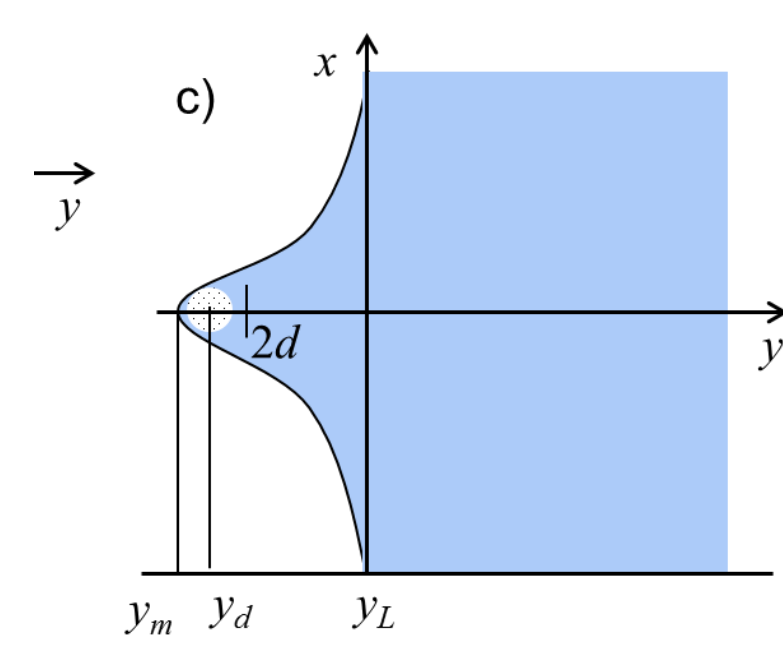

$$
\begin{gathered}
F_{l} \Delta y=\phi \Delta y\left(R_{i} \sin \alpha\right) W \\
\phi=\text { defect density }
\end{gathered}
$$

a)

Considering the case of isolated defects of size $d$

$$
W=\frac{1}{2} K u_{m}^{2}=\frac{f_{m}^{2} \ln (L / d)}{\gamma \pi \sin ^{2} \alpha} \text {. }
$$

In the case of surface roughness, instead, the specific energy $W$ of a topographical defect of size $d$ and height $h$ can be written as: 39

$$
\begin{aligned}
W=\frac{\gamma d^{2} \sin ^{2}\left(\frac{\mathrm{d} h}{\mathrm{~d} y}\right) \ln (L / d)}{2 \pi} . \\
\cos \alpha_{R}-\cos \alpha_{A}=\phi 2 W,
\end{aligned}
$$




\section{Second Summary Part 2: Macroscopic wetting}

- Wenzel Model for rough or structured surfaces

- Cassie Model for heterogeneous surfaces

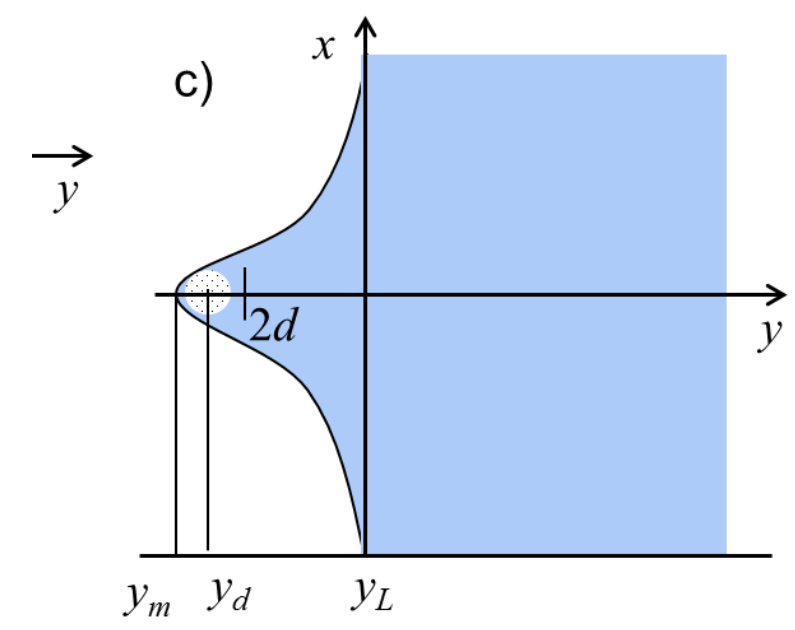

- Role of surface defects

- Contact angle Hysteresis
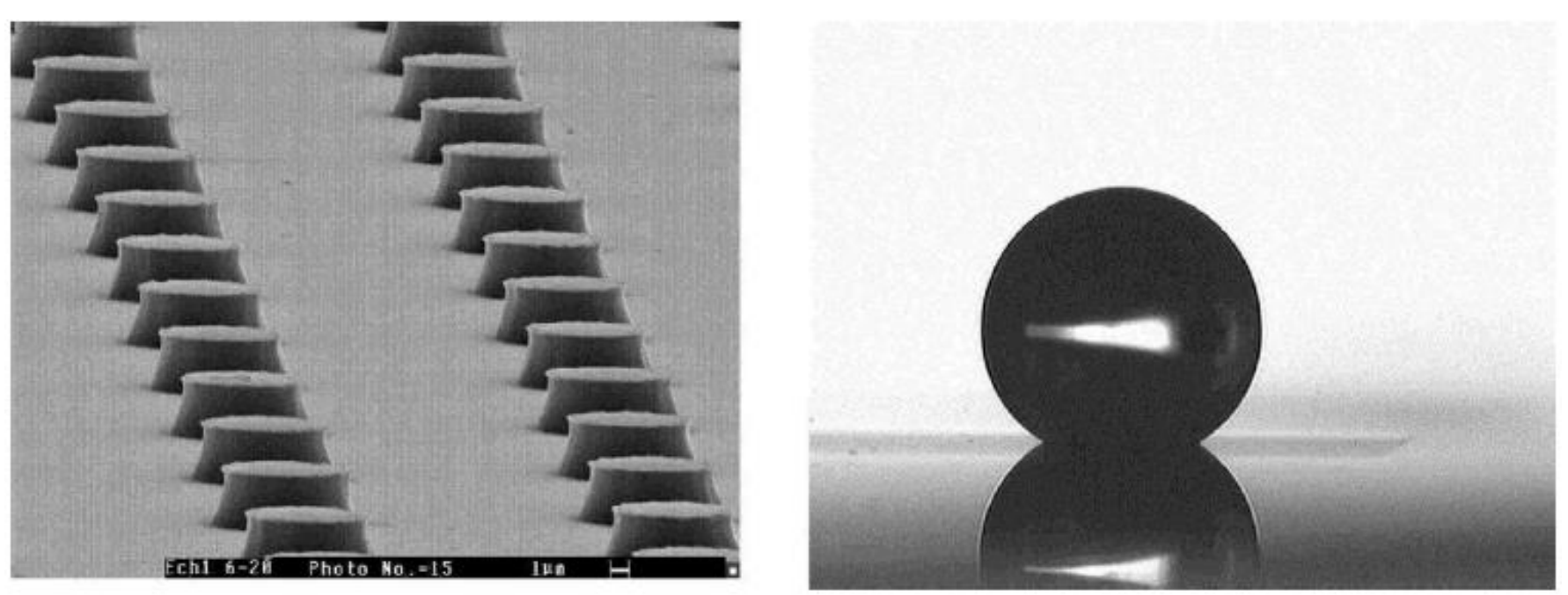
Fine tuning of

interactions

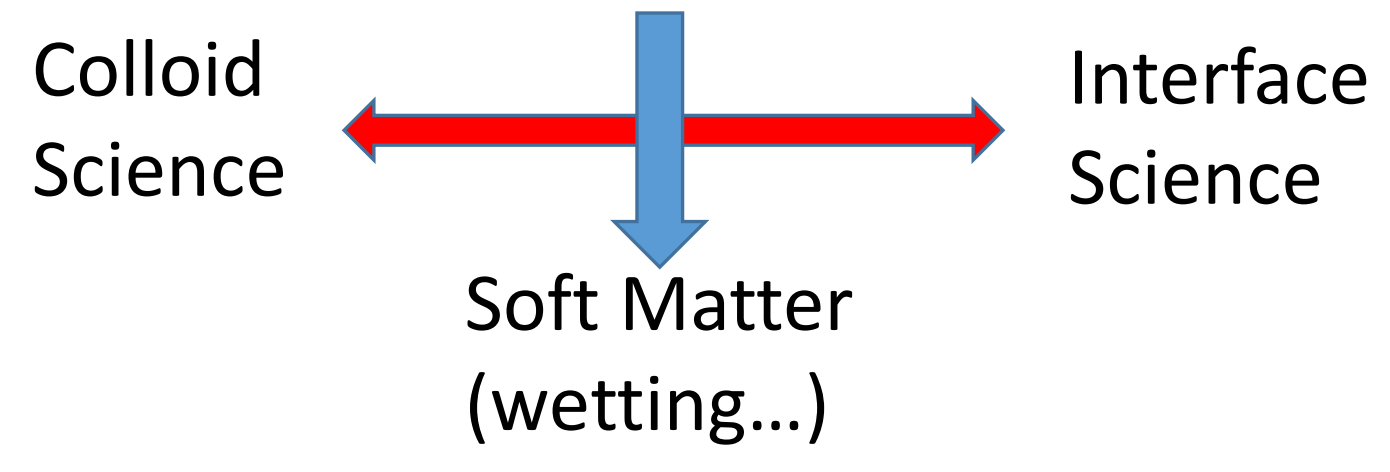

1. Concepts (surface tension, disjoining pressure, adsorption)

2. Wetting (contact angle, line tension, Wenzel and Cassie models)

3. (De)stabilization mechanisms in dispersed systems (gas in liquid: Foams) 
Foams:

gas bubbles

in a liquid

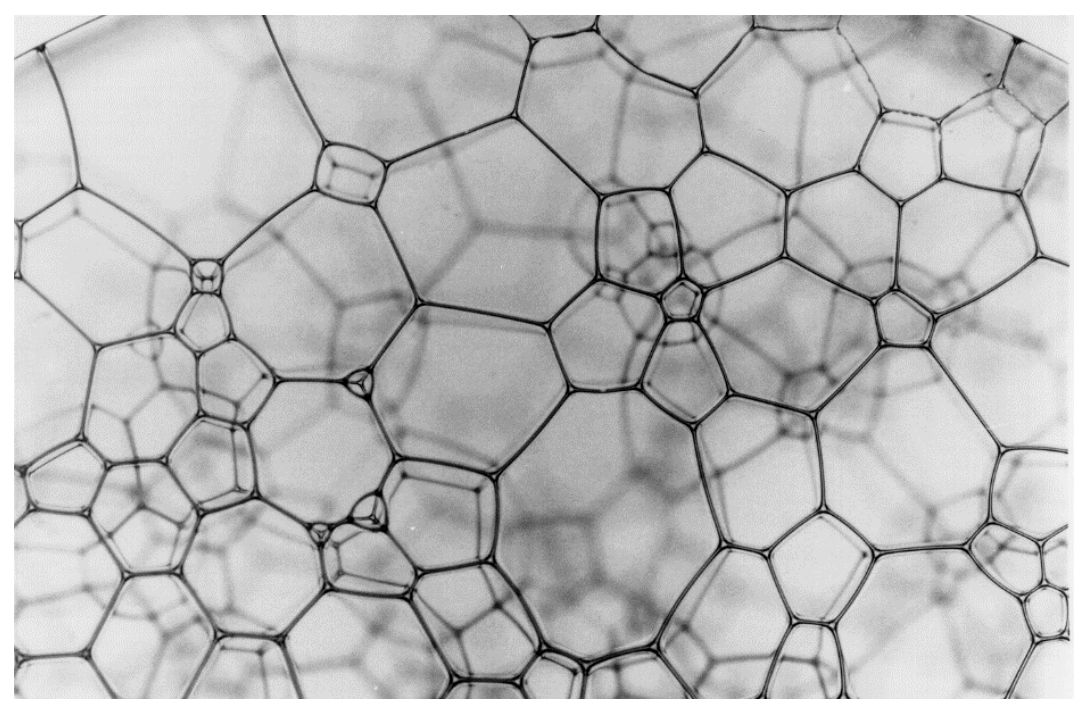

- Vertex

- region where four Plateau borders meet

- the point shared by four neighboring bubbles

- Plateau border

- scalloped-triangular channel where three films meet

- the edge shared by three neighboring bubbles

\section{Plateau border}
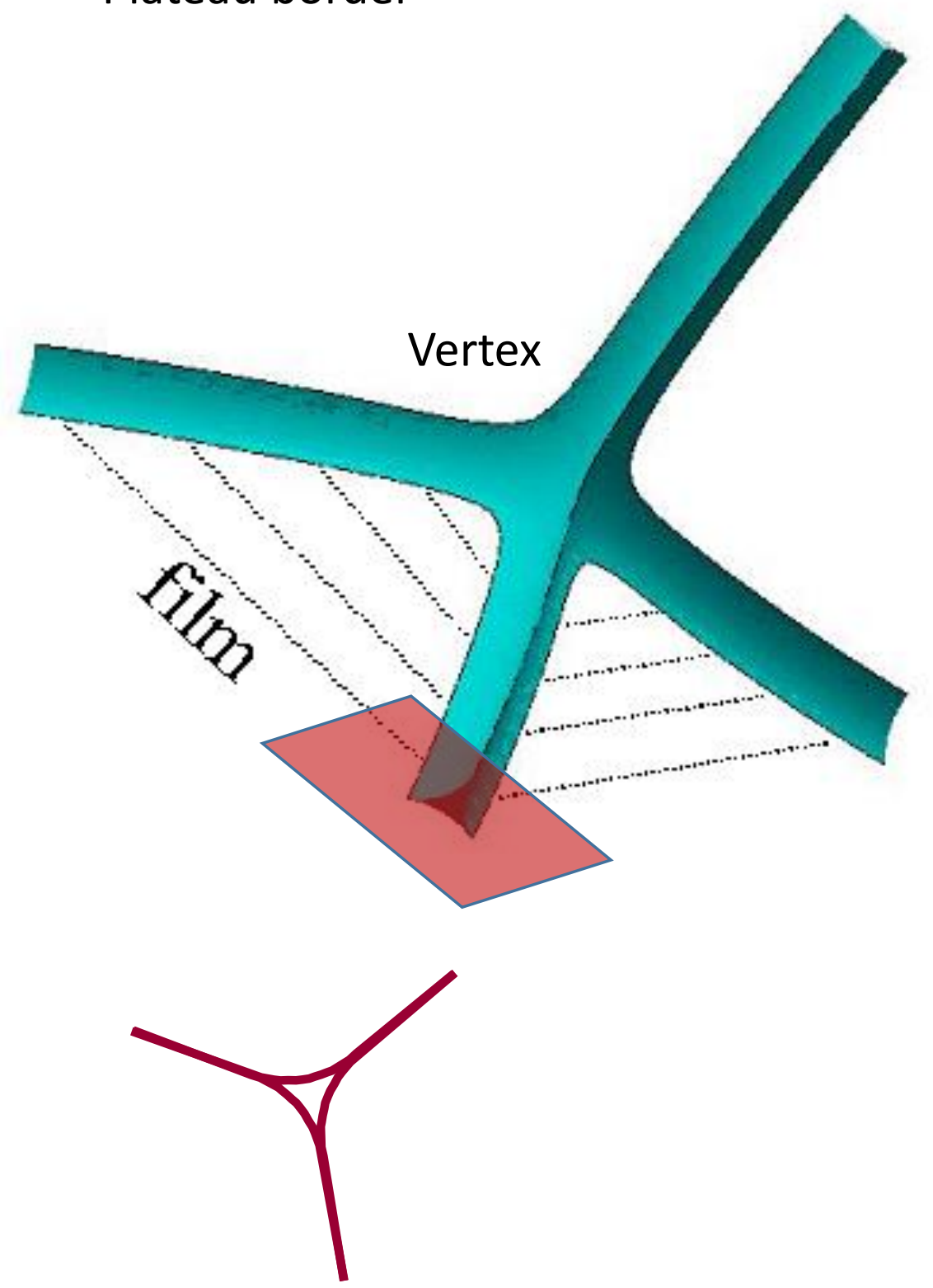


\section{Plateau's rules for dry foams}

- for mechanical equilibrium:

- i.e. for zero net force on a Plateau border,

- zero net force on a vertex,

- and $\Sigma \Delta \mathrm{P}=0$ going around a closed loop:

(1) films have constant curvature $\&$ intersect three at a time at $120^{\circ}$

(2) borders intersect four at a time at $\cos ^{-1}(1 / 3)=109.47^{\circ}$

- rule \#2 follows from rule \#1

- both are obviously correct if the films and borders are straight:
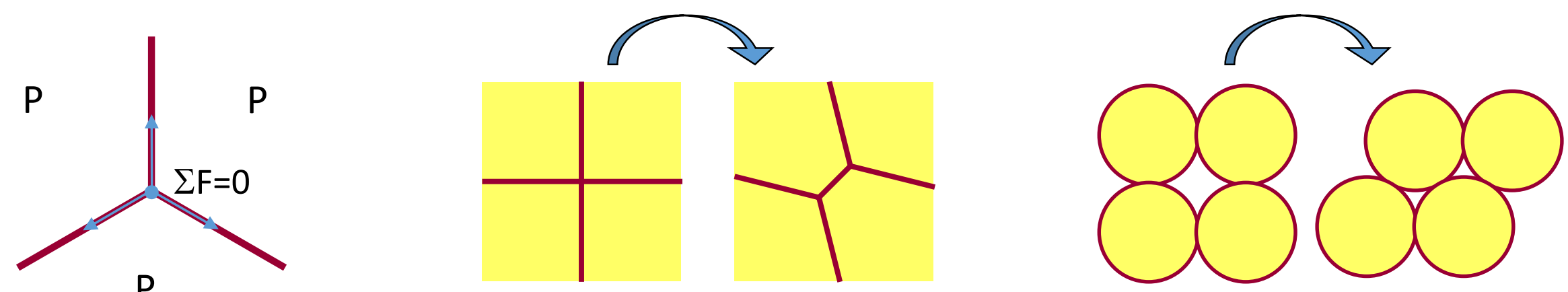


\section{Liquid distribution}

- division of liquid between films-borders-vertices

- Repulsion (between interfaces) vs surface tension

- wet vs dry
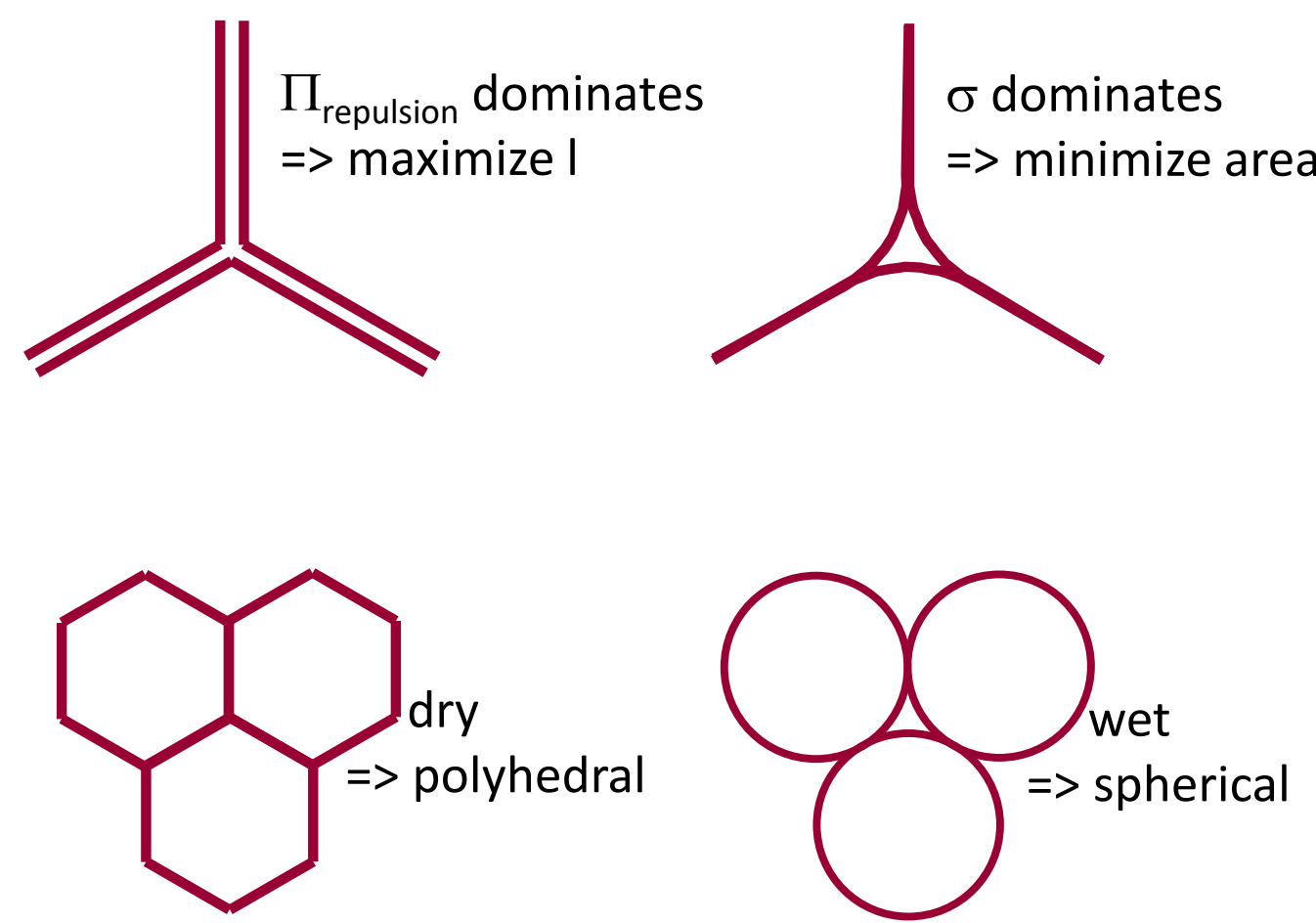

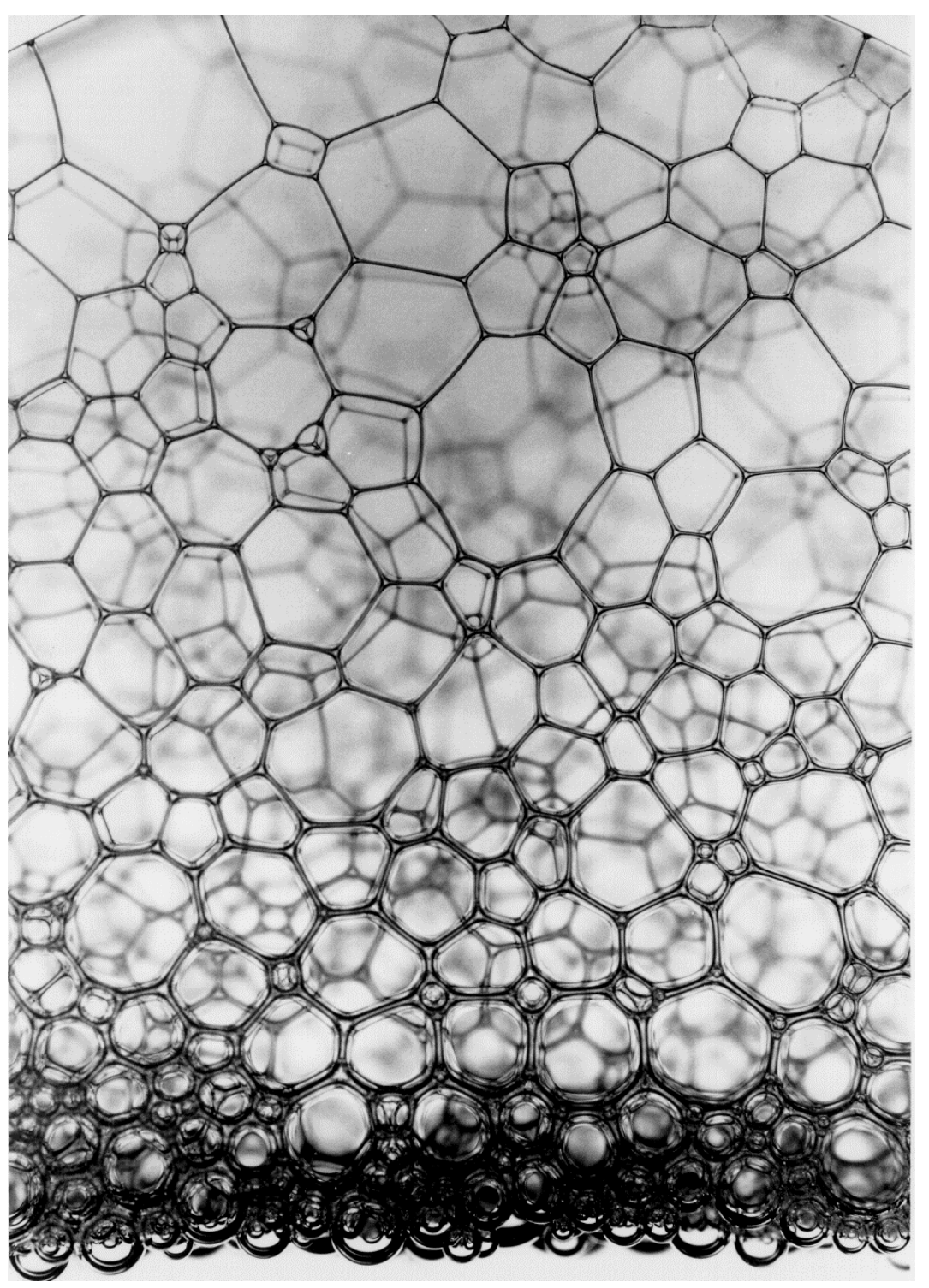




\section{Disjoining pressure}

Free energy of a foam film (suspended film L)

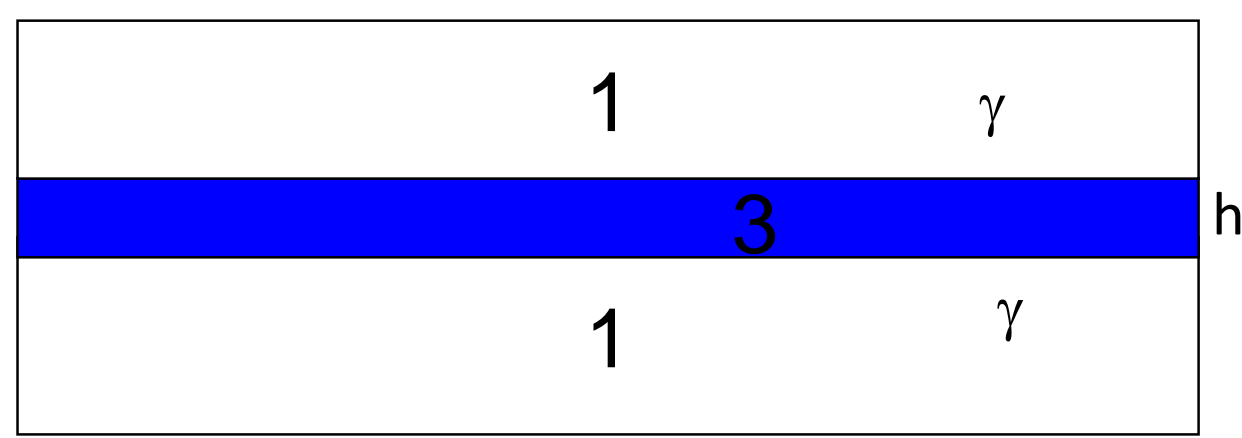

$$
E(h)=\gamma+\gamma+P(h)
$$

Disjoining pressure $\quad \Pi(h)=-\frac{d P}{d h}$

Energy per unit area $\quad V_{V d W}=-\frac{A_{H}}{12 \pi h^{2}} \quad$ Attractive

\section{$1-30 \mathrm{~nm}$}

$V_{E D L}=\frac{64 k_{B} T c_{\text {ion,bulk }}}{\kappa}\left(\tanh \frac{e \psi_{0}}{4 k_{B} T}\right)^{2} \exp (-\kappa h) \begin{aligned} & \text { Repulsive } \\ & \text { By surfactants }\end{aligned}$

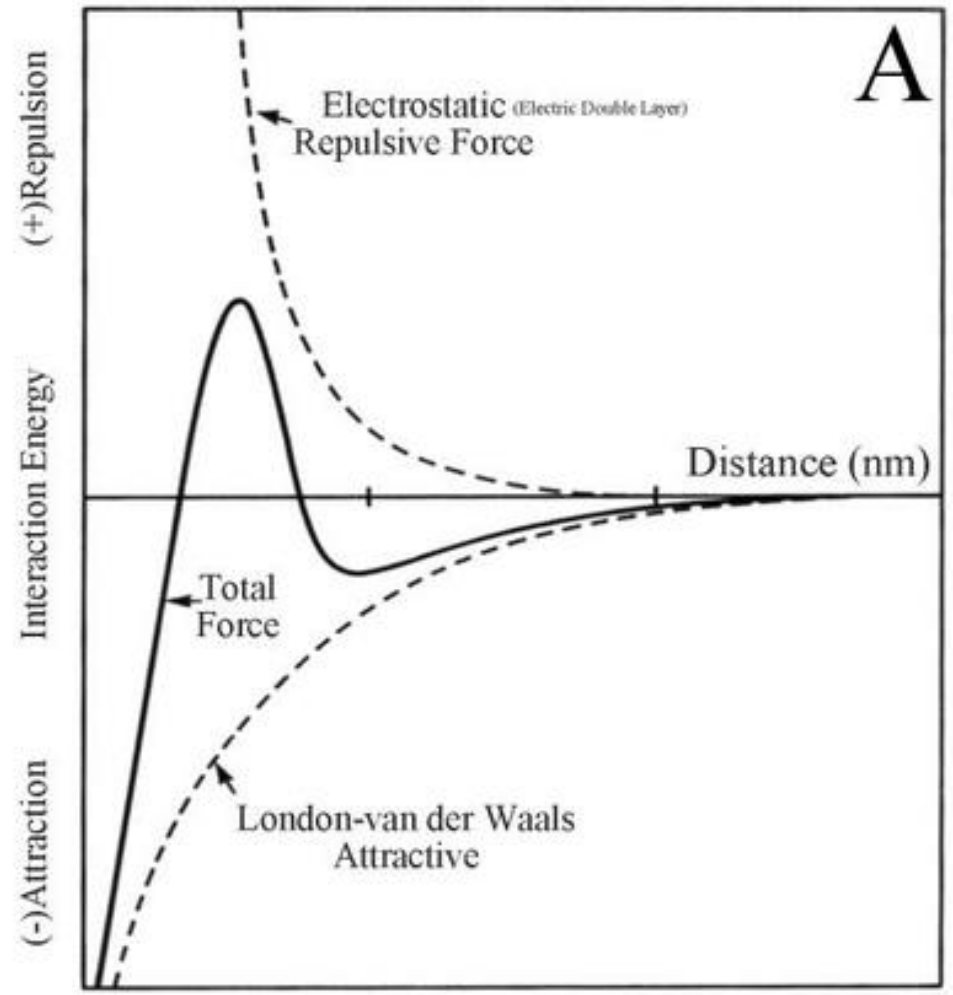




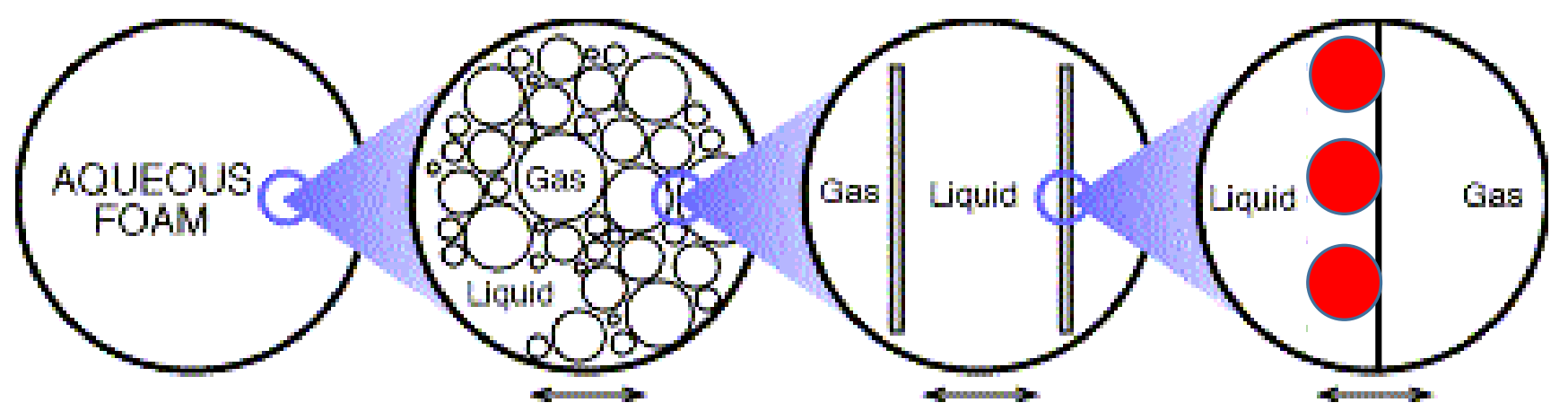

Macroscopic

Bubbles<smiles>C1CC2CCCC3CCCC(C1)C23</smiles>

\subsection{1}

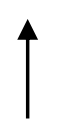

Liquid fraction $=\varepsilon=\mathrm{V}($ liquid $) / \mathrm{V}($ foam $)$

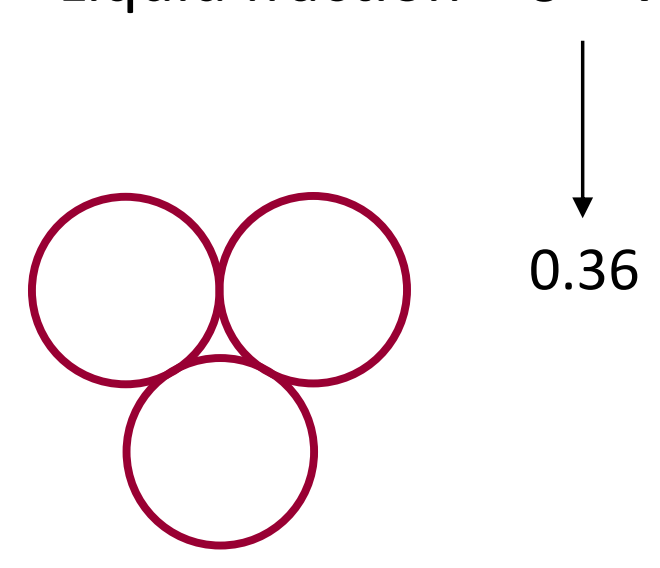

Films

$0.1 \mu \mathrm{m}$

$10 . . .100 \mathrm{~nm}$ Interface
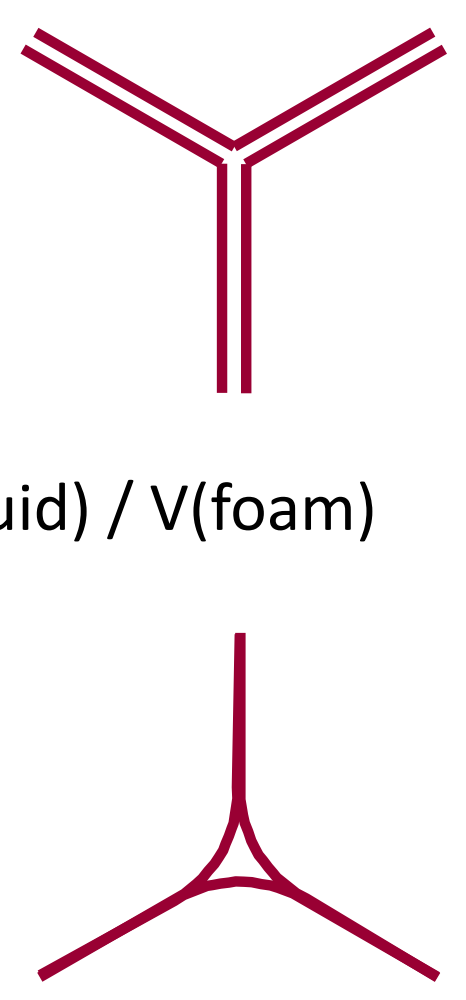

Interfaces can be stabilised by surfactants, polymers or particles.

The latter are the corresponding "Pickering"

Aqueous Foams 
Foam Destabilisation Mechanisms (Dynamics)

- Drainage (gravity): decrease of $\varepsilon$

- Coalescence: van der Waals, $-\mathrm{A}_{H} / 12 \pi d^{2}$

- Coarsening
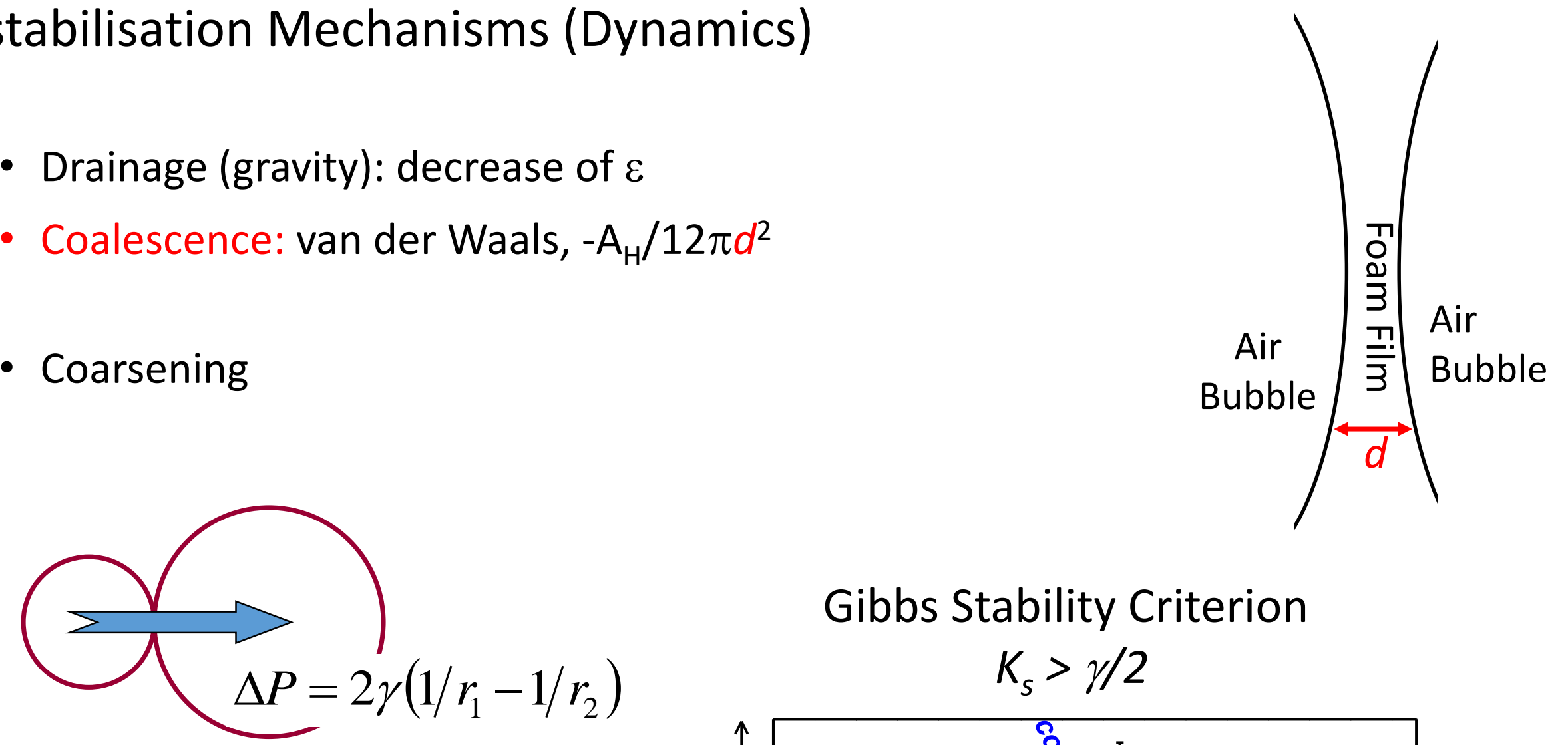

$d P / d R=-\gamma / R^{2}+2 K_{s} / R^{2}$

$P=$ bubble pressure

$R=$ bubble radius

$\gamma=$ surface tension

$K_{s}=d \gamma / d \ln (A)=$ surface elasticity

Gibbs Stability Criterion

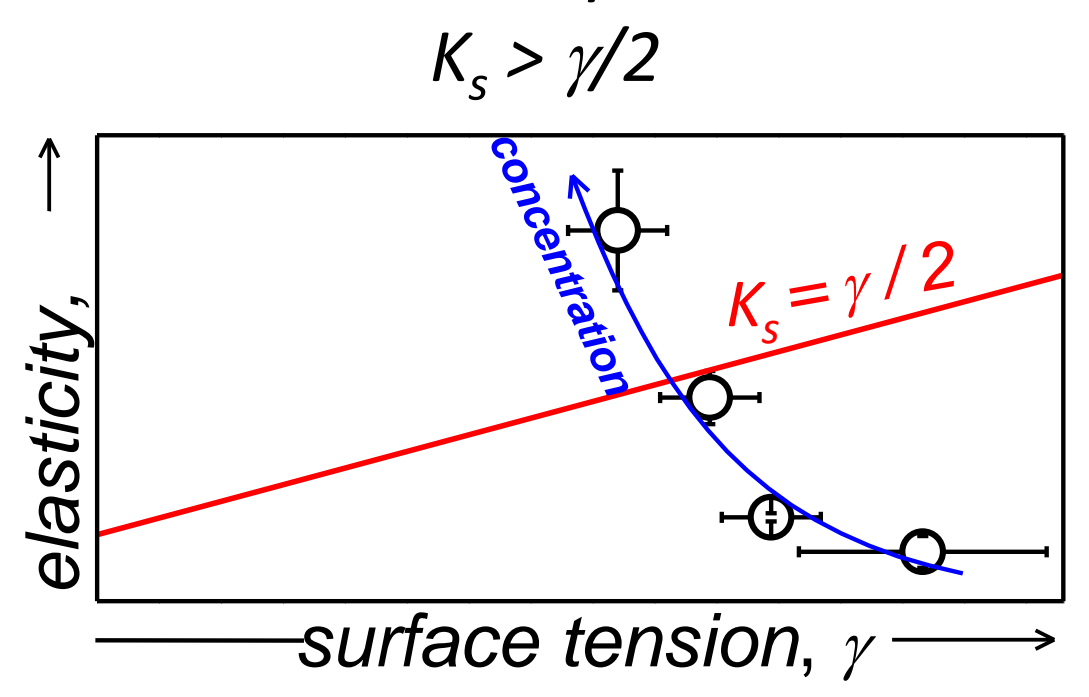


Colloidal scale

Macroscopic scale a Binks BP
Oil + water

Oil-in-water emulsion

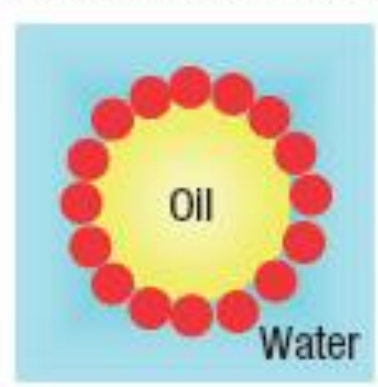

Hydrophilic particle

$\theta=0^{\circ}$

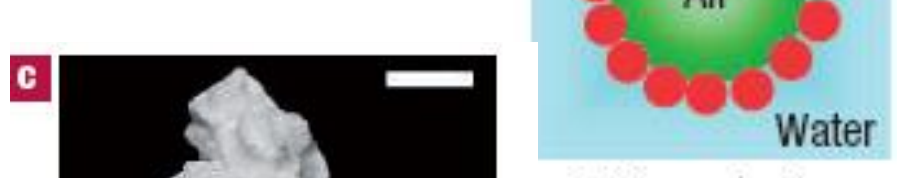

Air-in-water foam Air- + Water

Water-in-oil emulsion

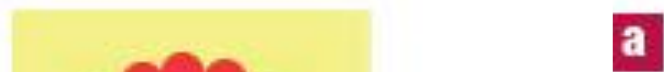

Silica NP, $70 \mathrm{~nm}$, silane treat.

$\mathrm{SiOH} \%$

$\begin{array}{lll:llllll:lll}\text { a } & 14 & 20 & 32 & 36 & 42 & 51 & 57 & 62 & 66 & 78 & 100\end{array}$
Hydrophobic

Water

Oil particle

$\vec{\theta}=180^{\circ}$

Water

Air b

Water-in-air powder

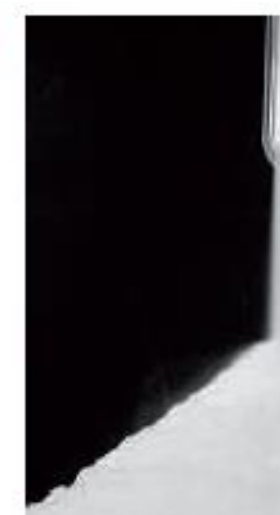

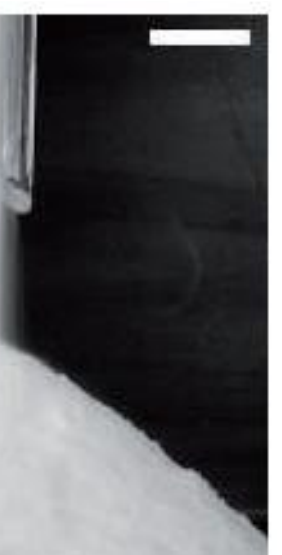

Contact angle of NP is $\alpha_{E}$ ? Spontaneous adsorption? Fully packing?

Contact angle induced curvature? 
1. Is it really just wetting (Young equation)?

2. How colloids attach onto the water interface?

Air (Foam)

Oil (Emulsion)

Water 

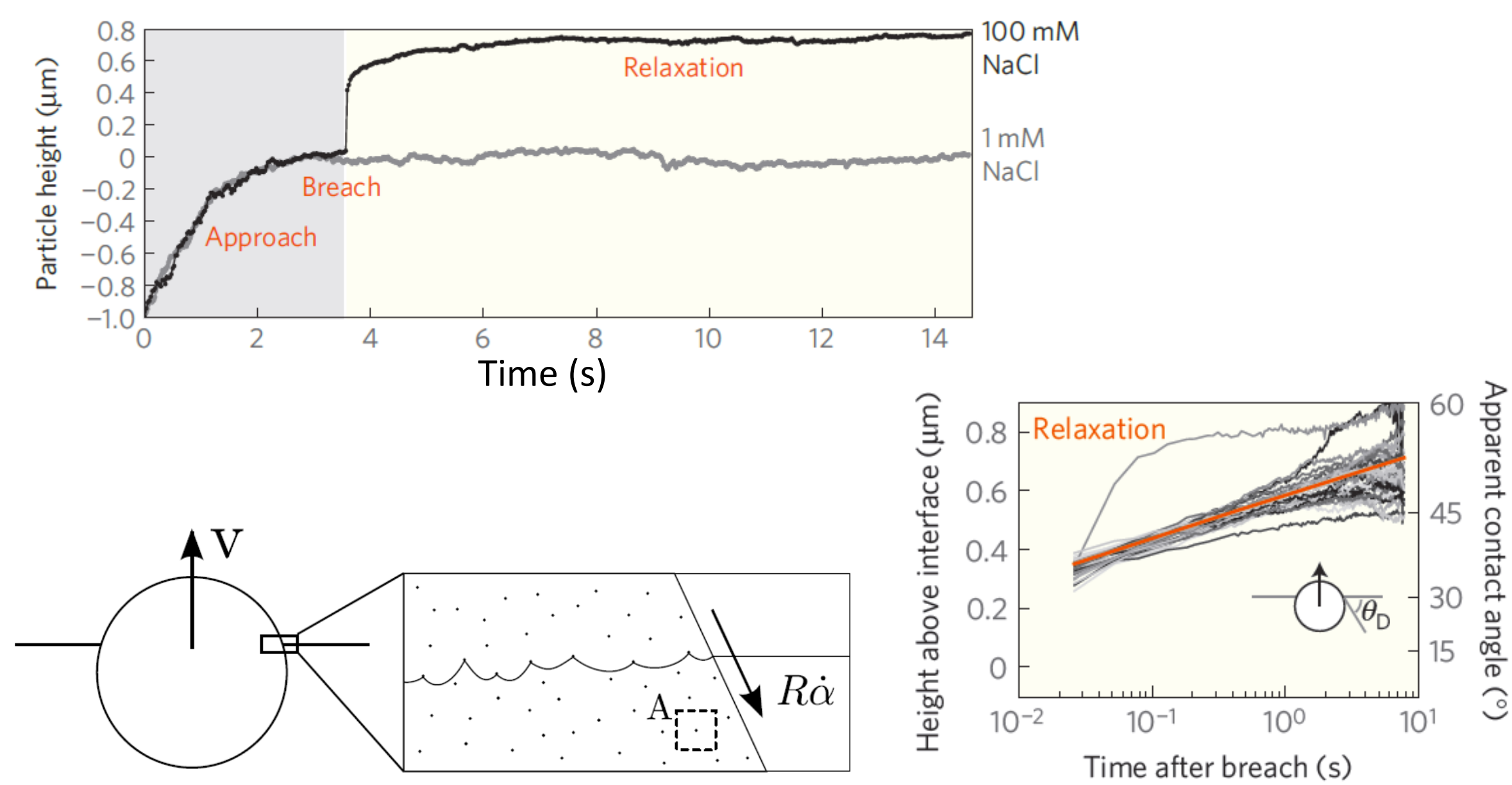

Thermal hopping of the contact line over surface defects 
From what we saw before
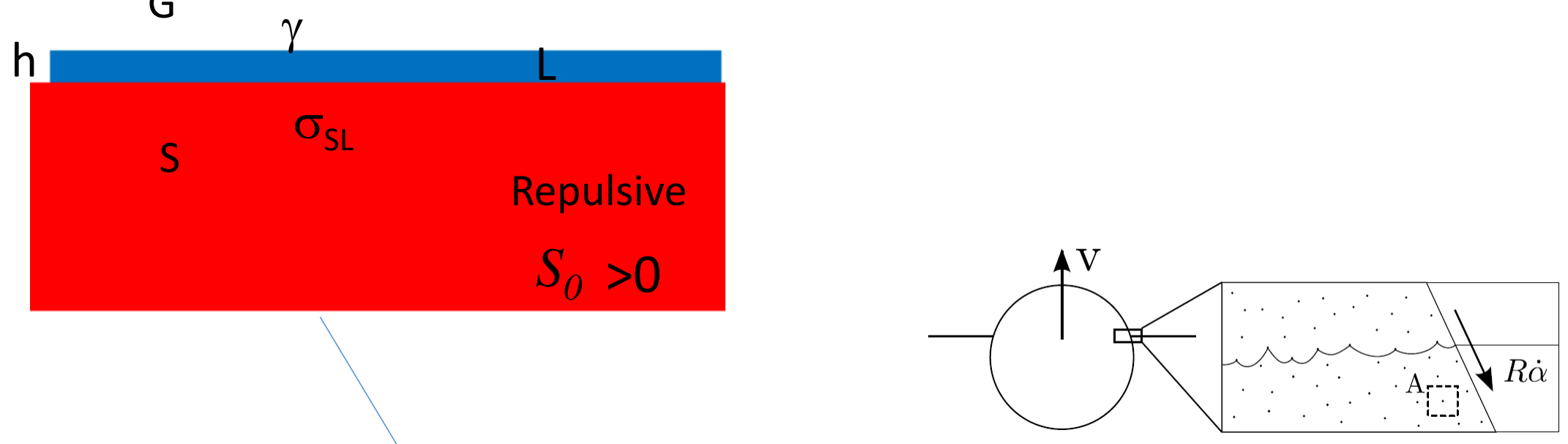

Wetting dynamics

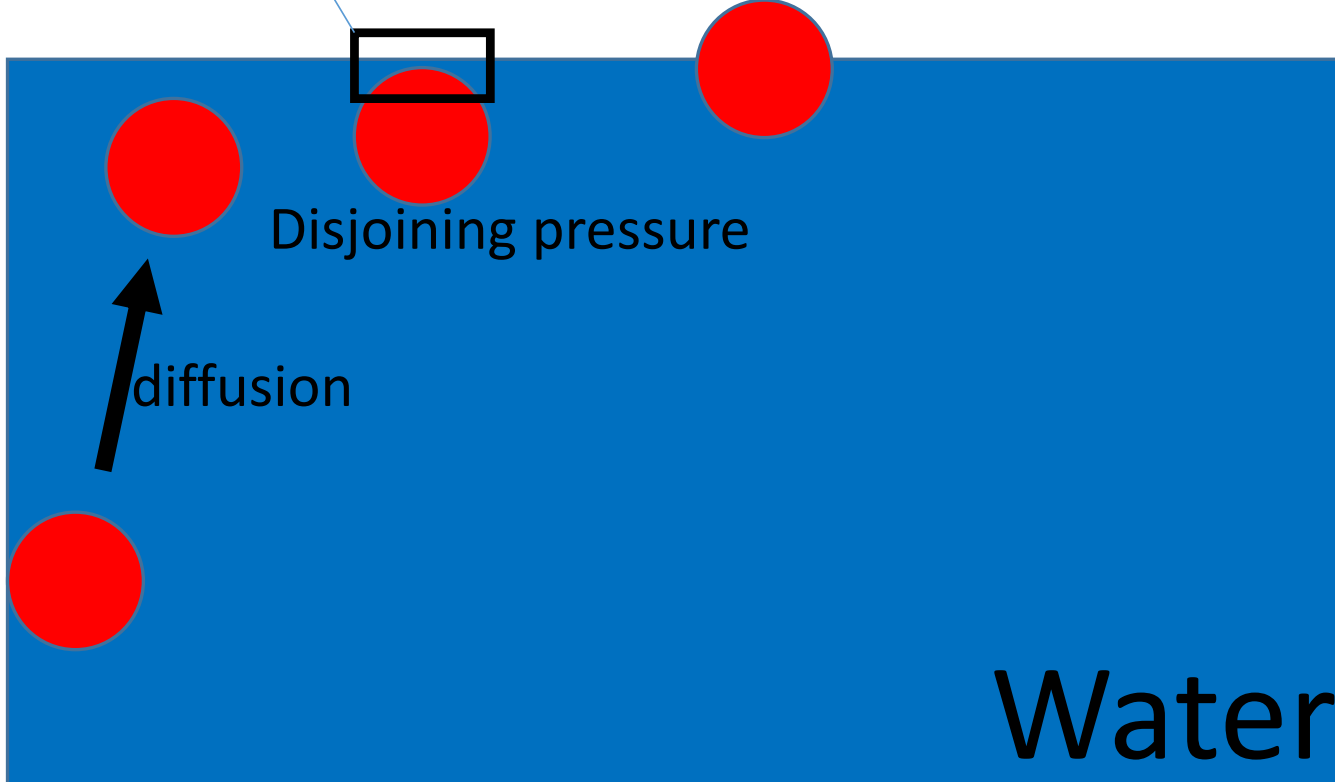


Contact angle of NP is $\alpha_{E}$ ? In general NO. $f($ size, roughness, line tension)

Spontaneous adsorption? In general NO. VdW and EDL are repulsive

Fully packing? To be investigated

Contact angle induced curvature? Not really 


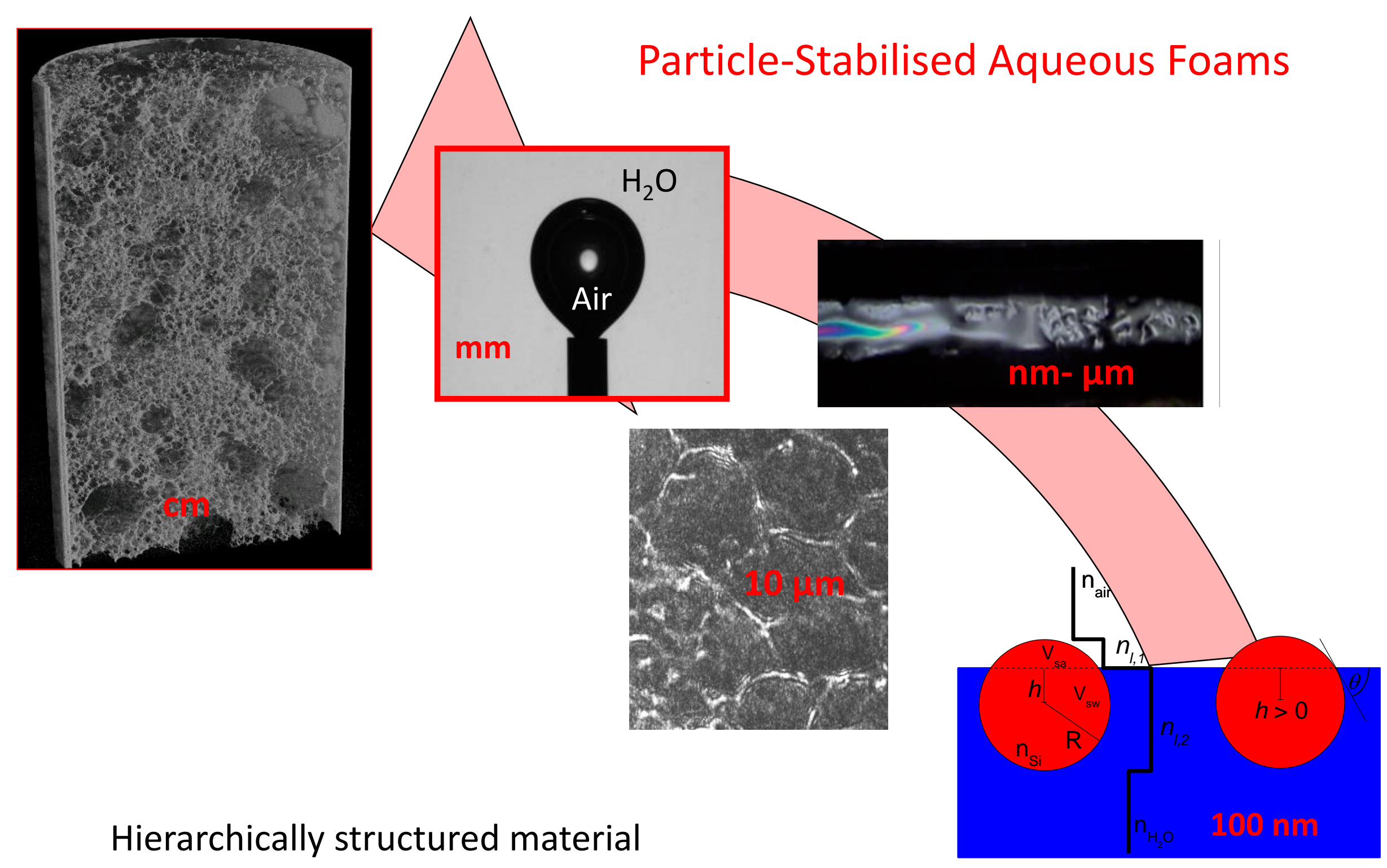




\section{Fumed Silica Particles}

elementary size $=20 \mathrm{~nm}$

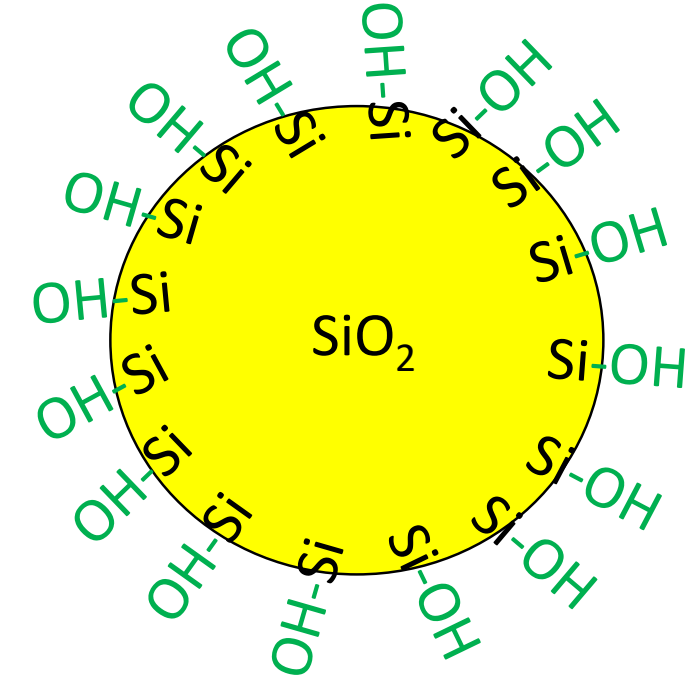

hydrophilic
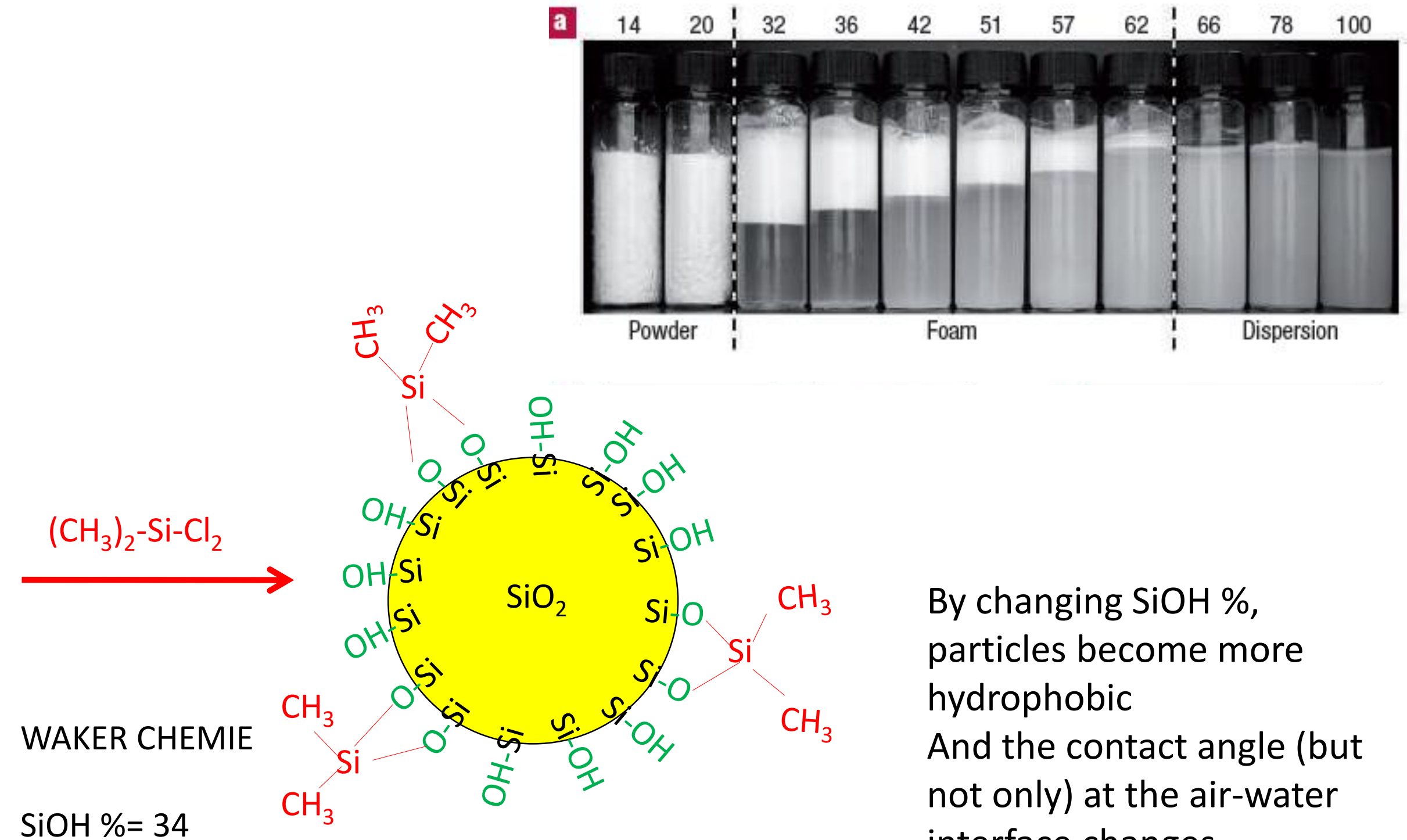

hydrophobic
By changing $\mathrm{SiOH} \%$, particles become more hydrophobic And the contact angle (but not only) at the air-water interface changes 


\section{"Pickering" -}

\section{Aqueous Foams}

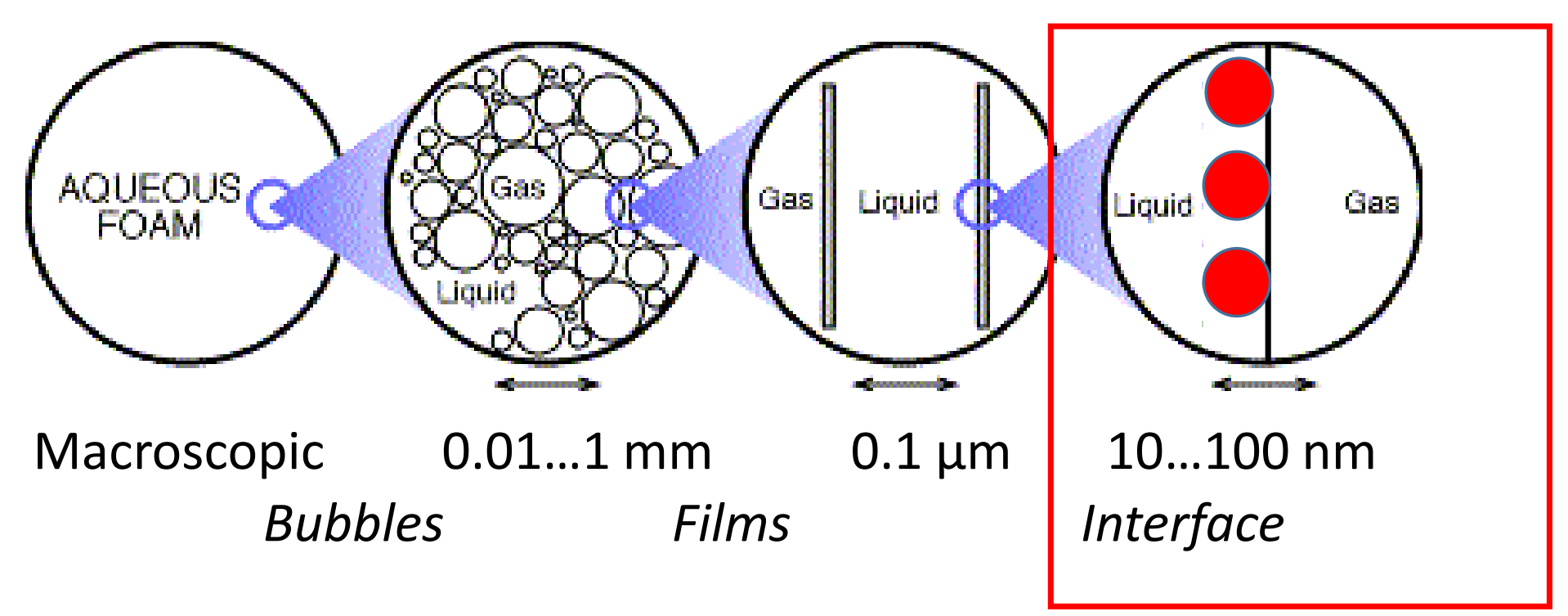


In situ-contact angle by Ellipsometry (or by X-ray, Neutron @ILL)

model

- refractive index are known

- Particle monolayer

- Spherical shape

- Effective media approx:

$\mathrm{n}_{\mathrm{L}, \mathrm{i}}=f$ (volume fraction)

3 parameters: $R, h, \phi$ $\alpha=\arccos (\mathrm{h} / \mathrm{R})$

2 fitting parameters

Spread layers,

$\Gamma$ known $[$ mass/area $]=f(\phi, \mathrm{R})$

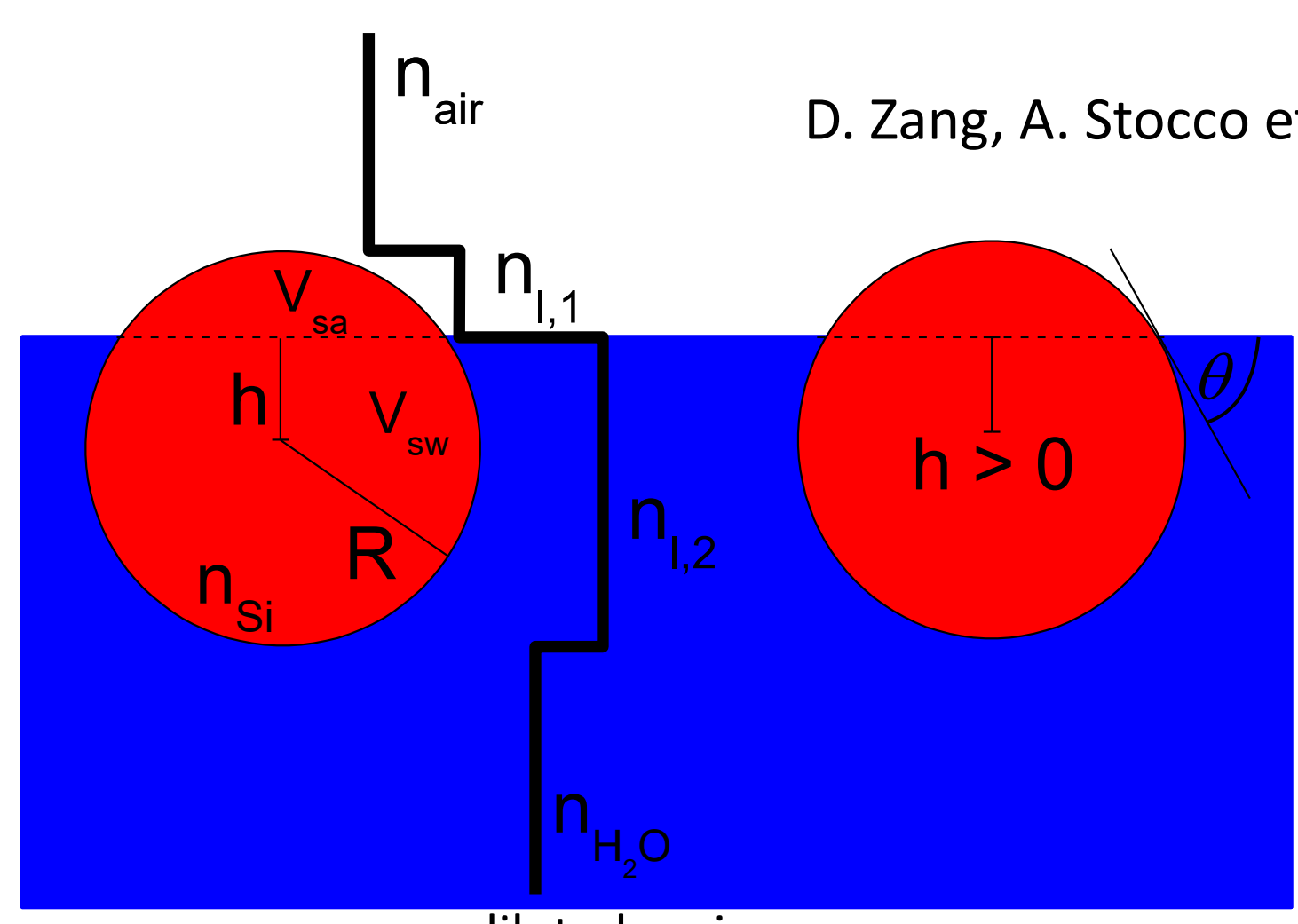

diluted regime

In-situ $\alpha$ measurements for spread particle layers!

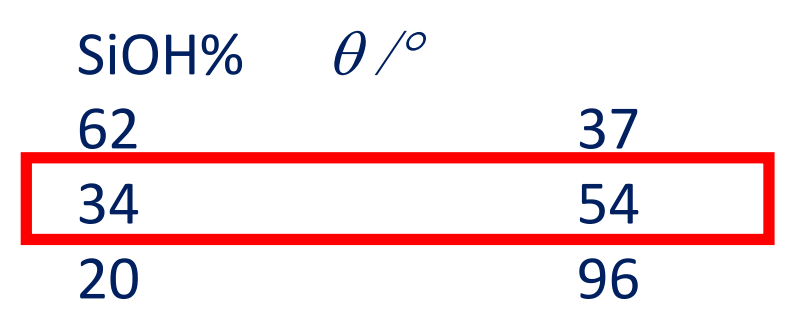




\section{Adsorbed Particle Layer}

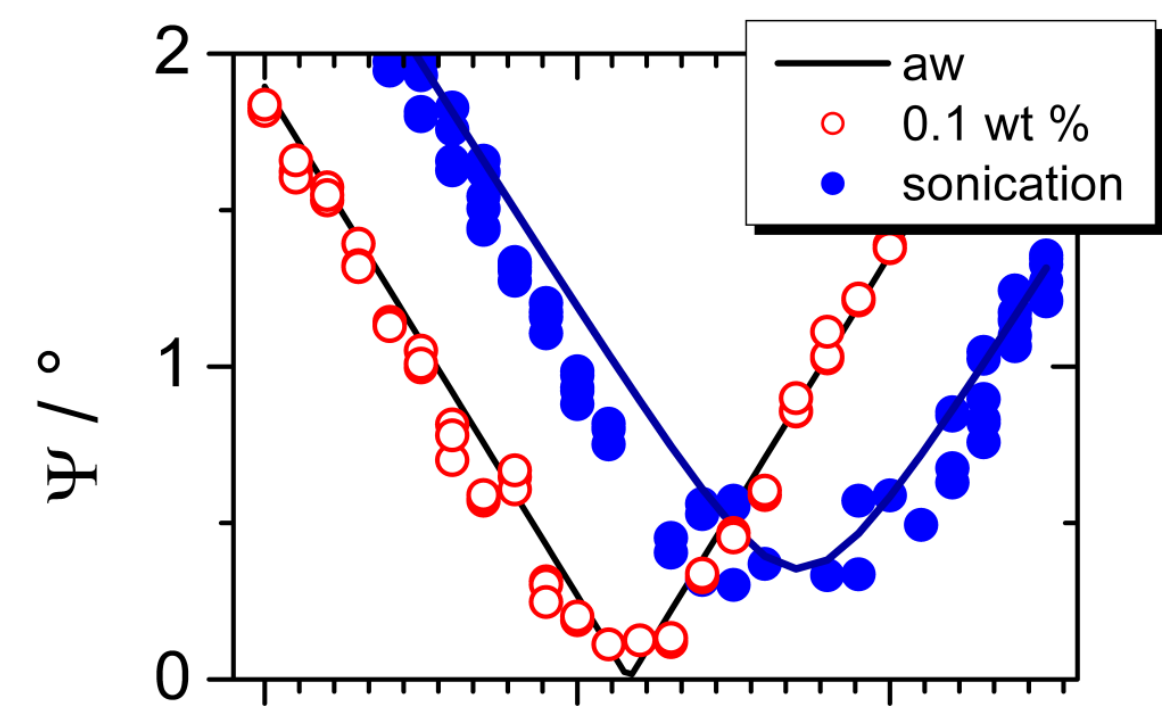

Energy input to overcome an Electrostatic barrier

NO full pack

Data are more scattered for adsorbed layer when compared to Spread layers

Brewster angle microscopy

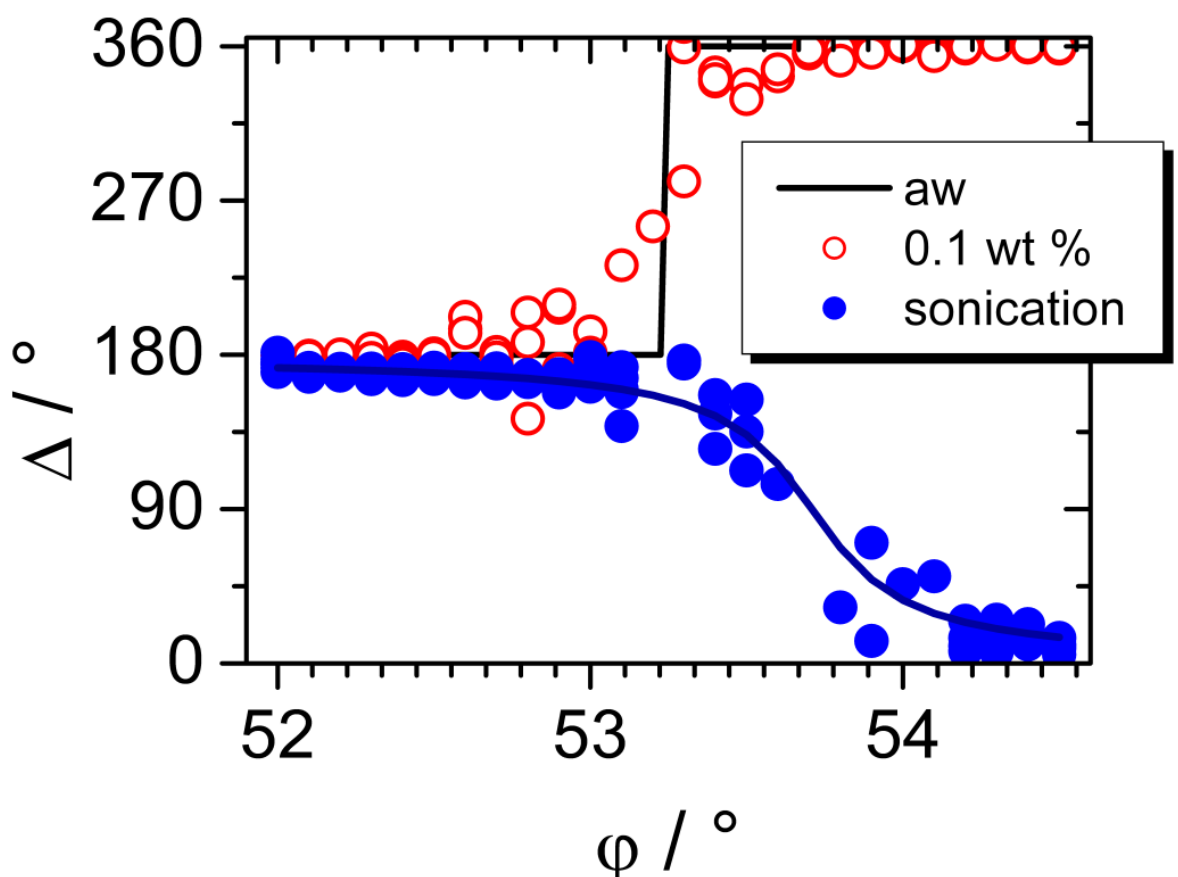

$\rightarrow$ Interfacial Network

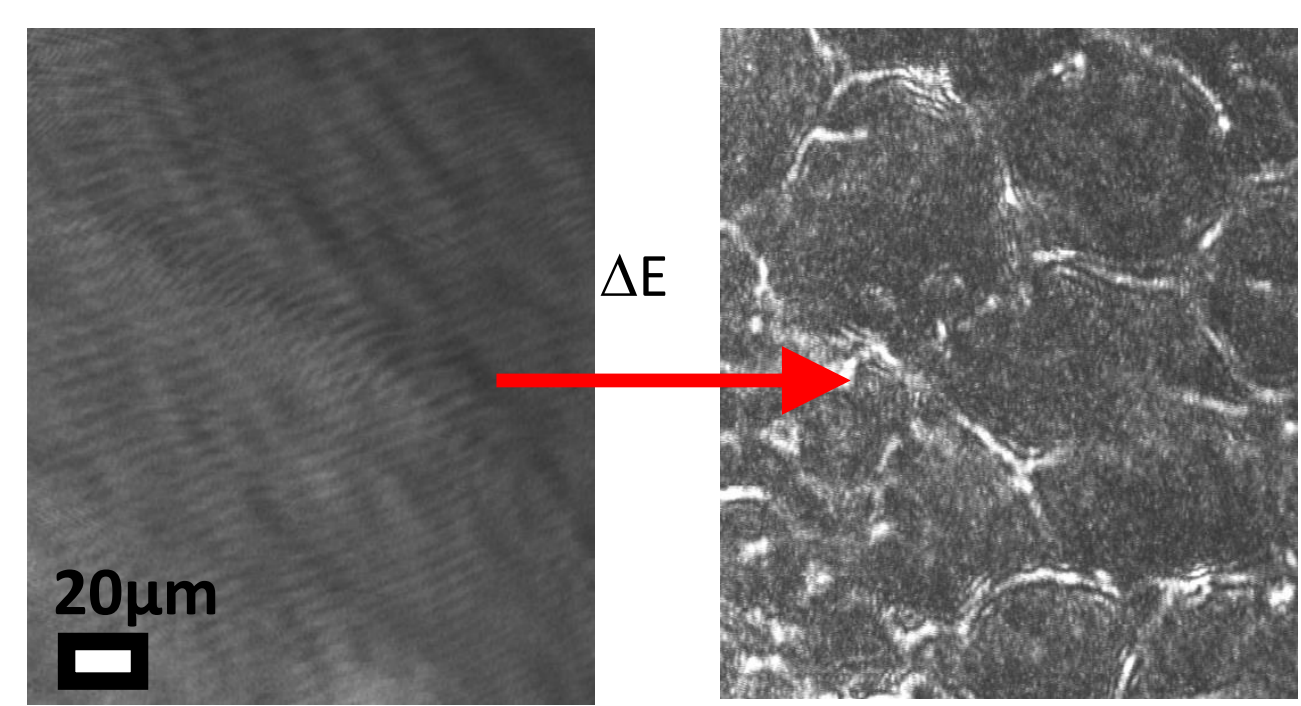


Surface Tension - $\gamma(\mathrm{c})$ water dispersions

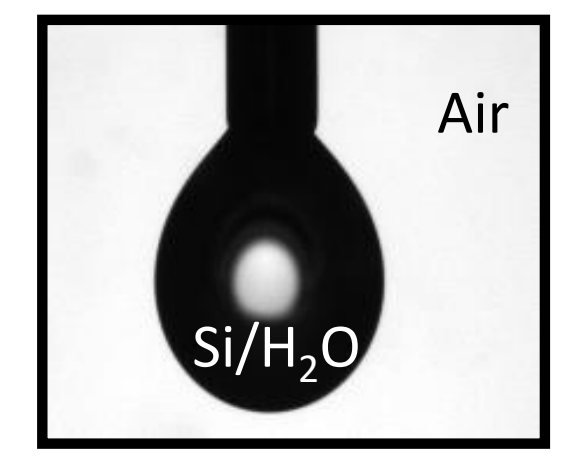

Pendant drop

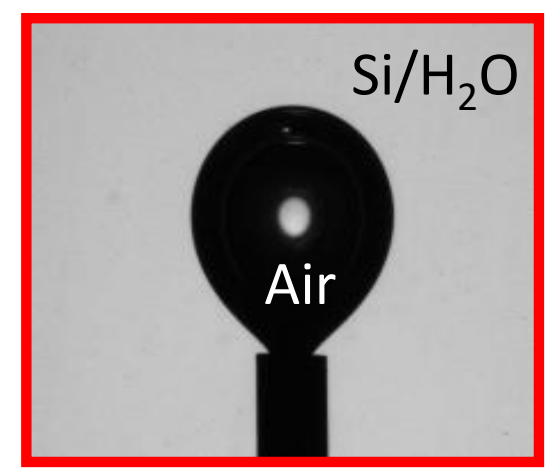

Rising bubble

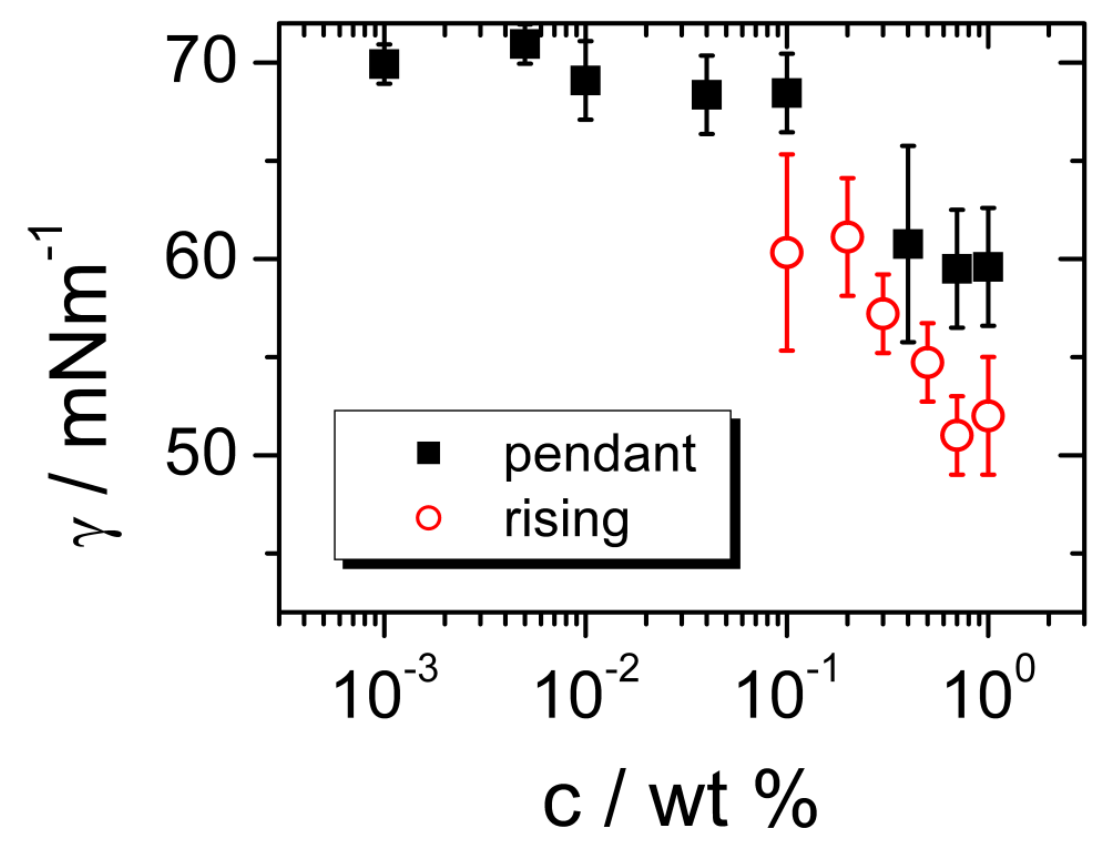

Groot and Stoyanov, Soft Matter 2010
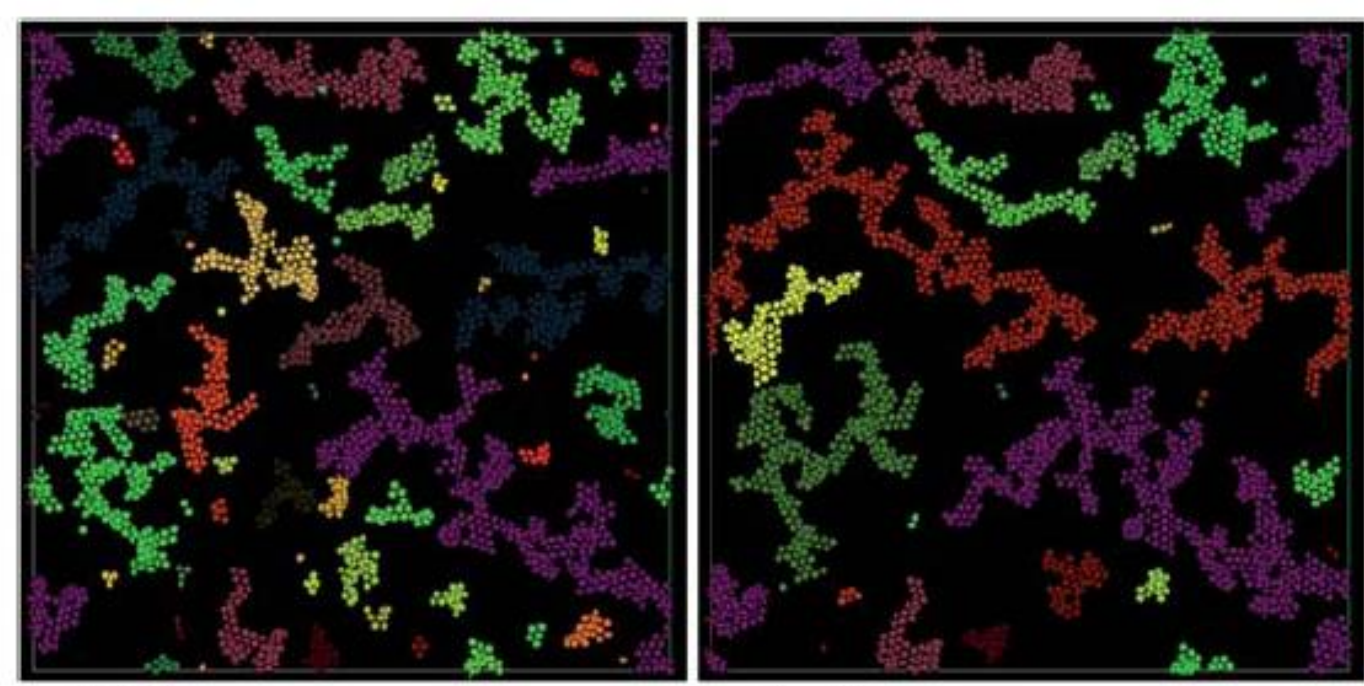

$t=48$

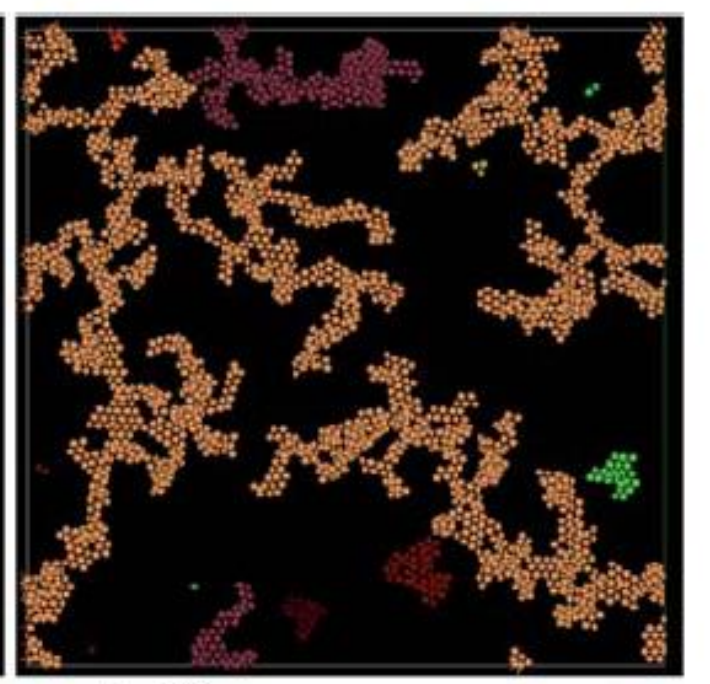

$\Delta \gamma=\Gamma^{10}$

2D percolation

- Fractal

- Particle network 
Surface Elasticity - Oscillating drop/bubble experiments

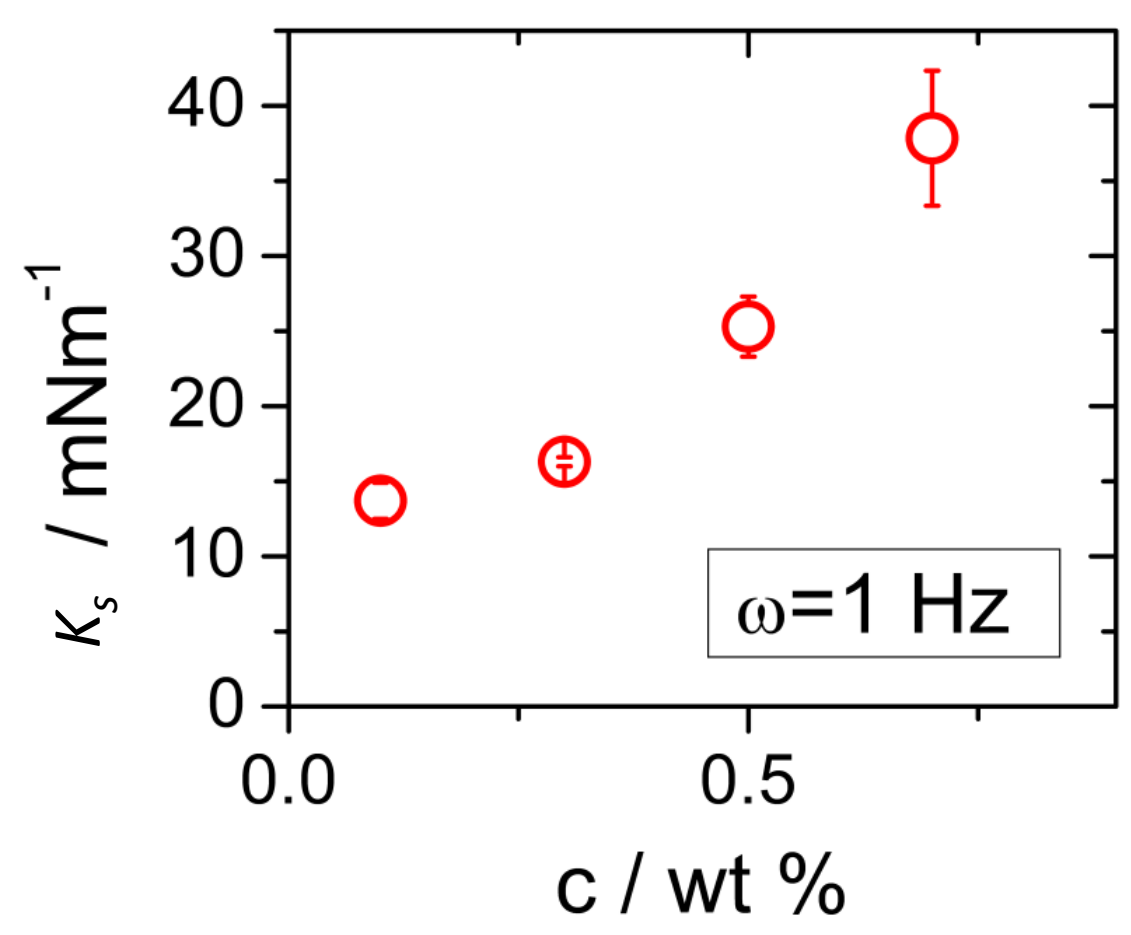

Interfacial (Bubble) study

By changing $c, K_{s}$ and $\gamma$ change Obtaining $K_{s}>\gamma / 2$

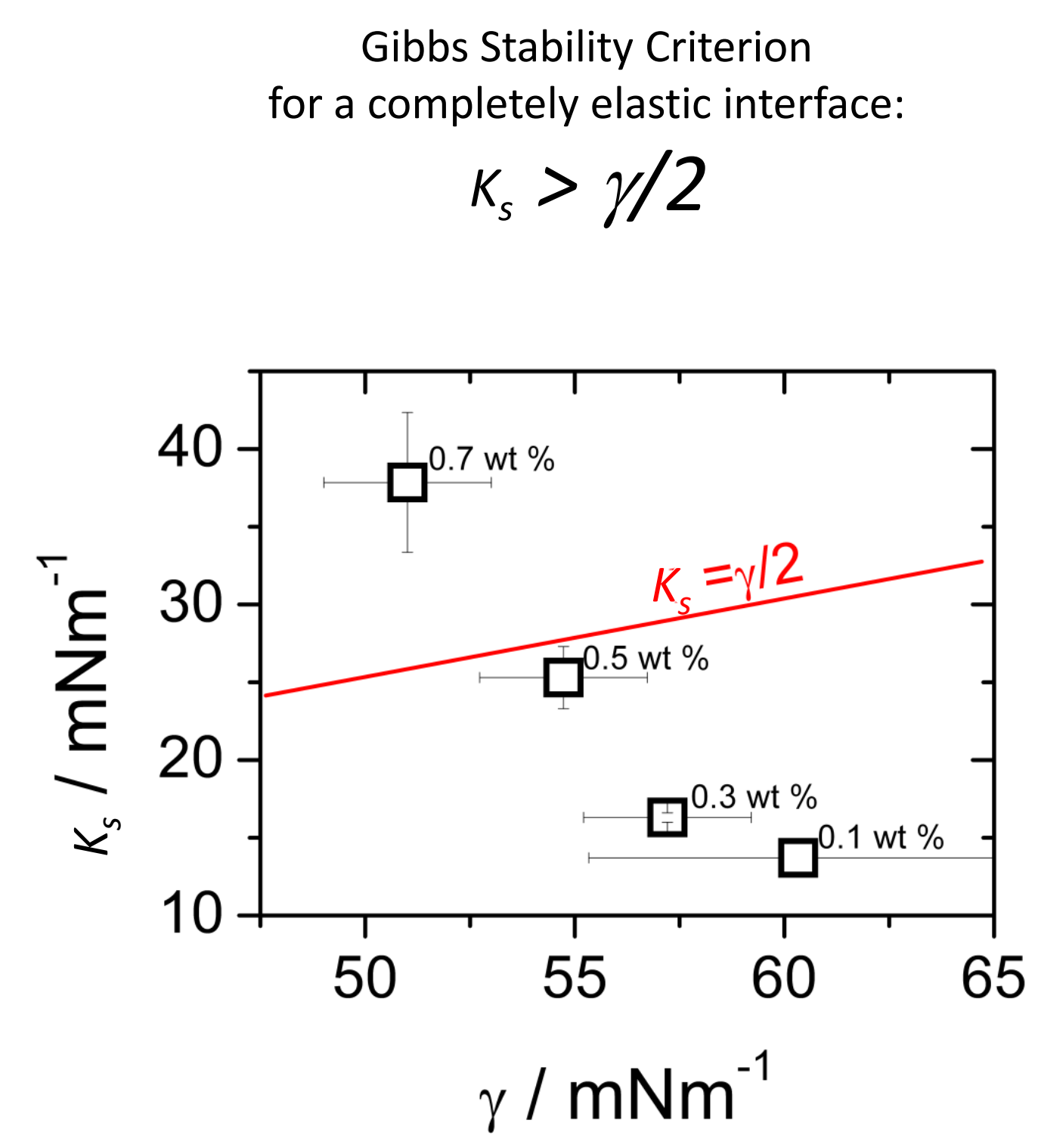


"Pickering" -

Aqueous Foams

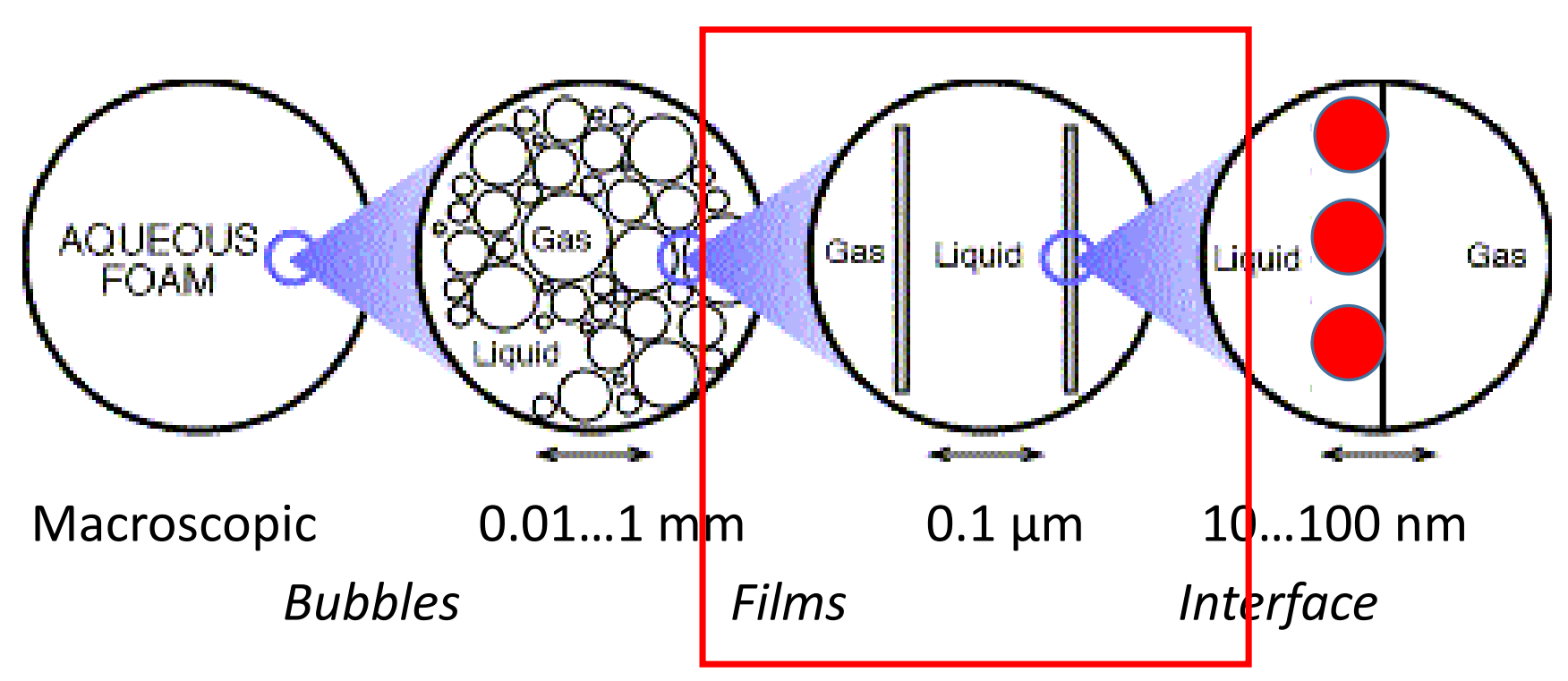


Free Suspended Foam Film

$V=5 \mu \mathrm{L}$

$2 \mathrm{~mm}$
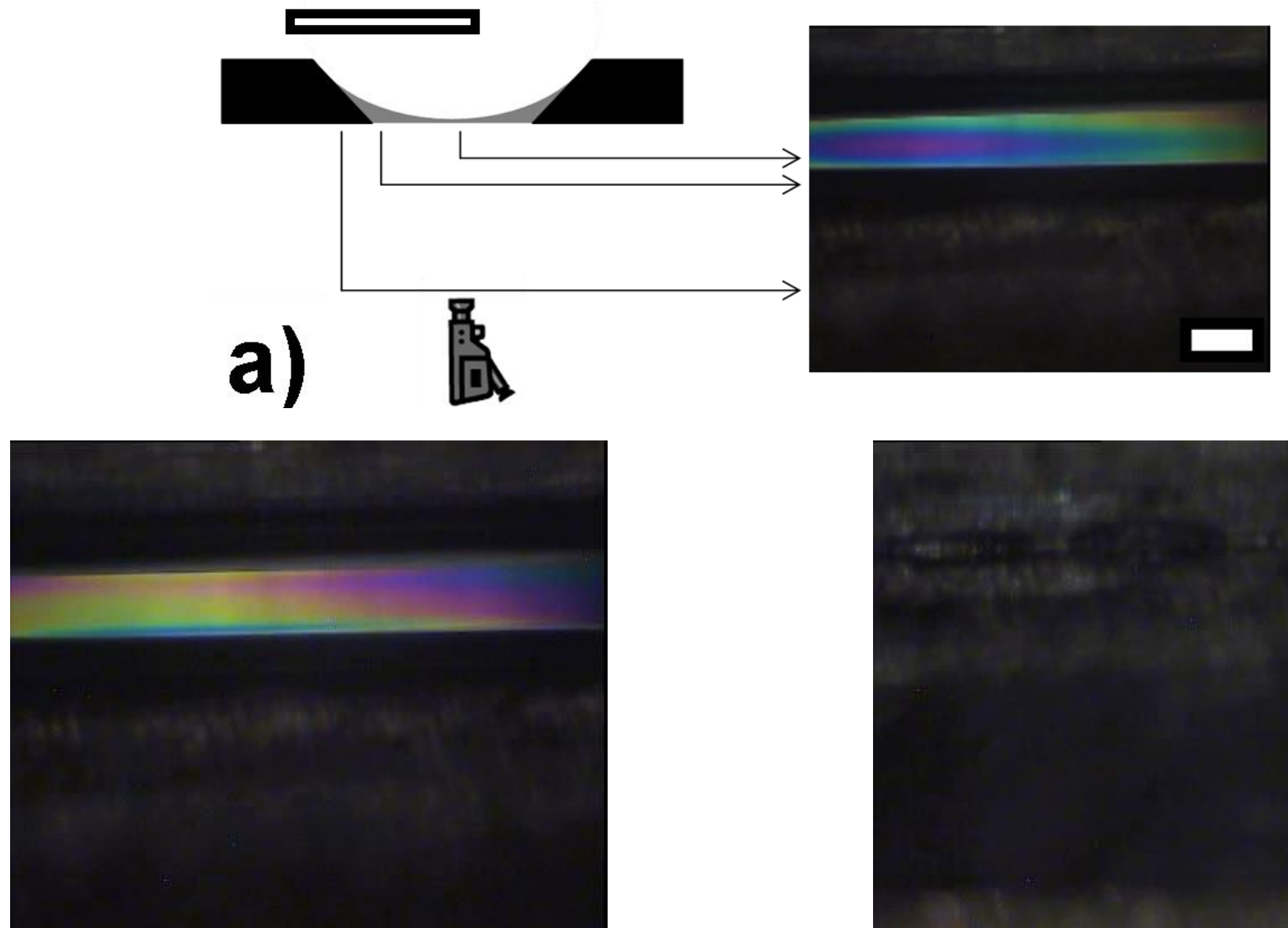
"Pickering" -

Aqueous Foams

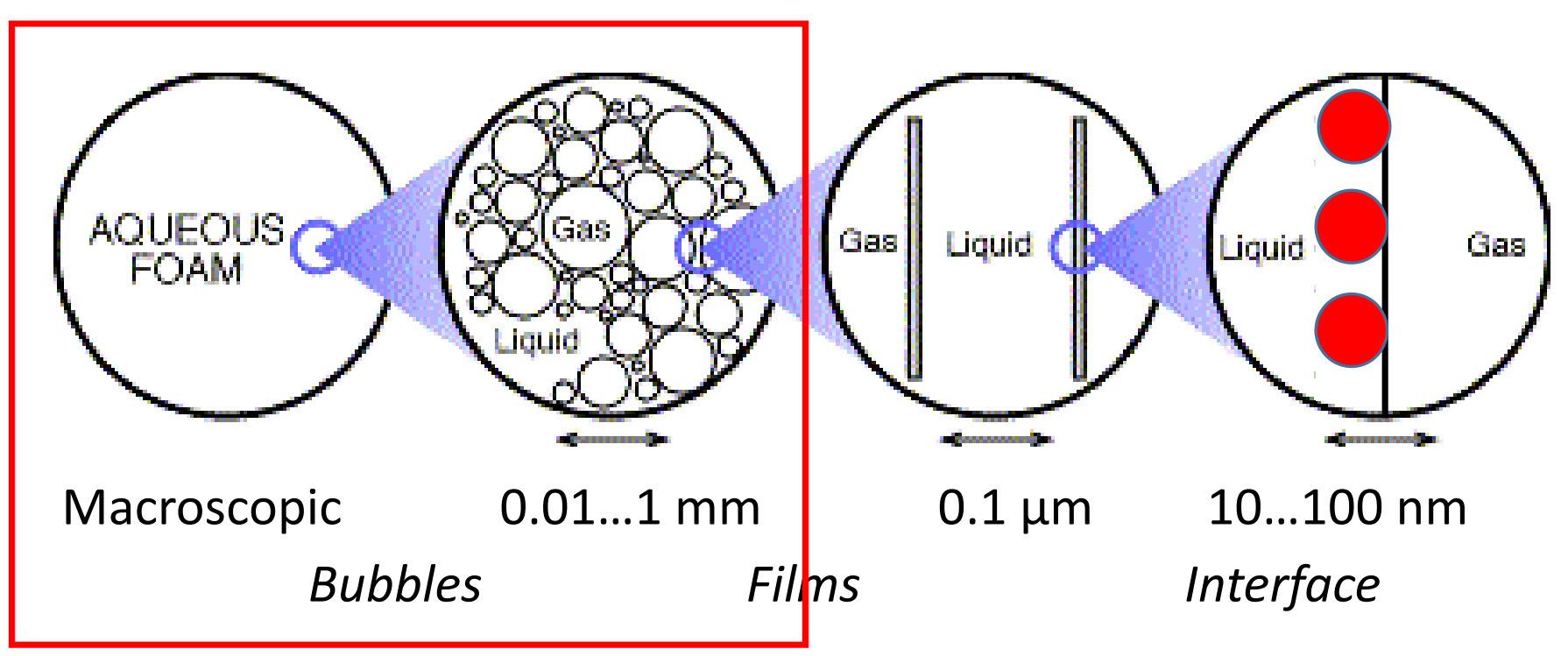


Fumed Silica aqueous foams production

Turbulent mixing DRY

- $\varepsilon=0.2-0.1$

- Bubble size $\mathrm{R}=10-70 \mu \mathrm{m}$

- Polydispersity $20 \%$

By shaking

WET

$\cdot \varepsilon=0.34-0.2$

- Bubble size $R=100-300 \mu m$

- High Polydispersity > 20\%

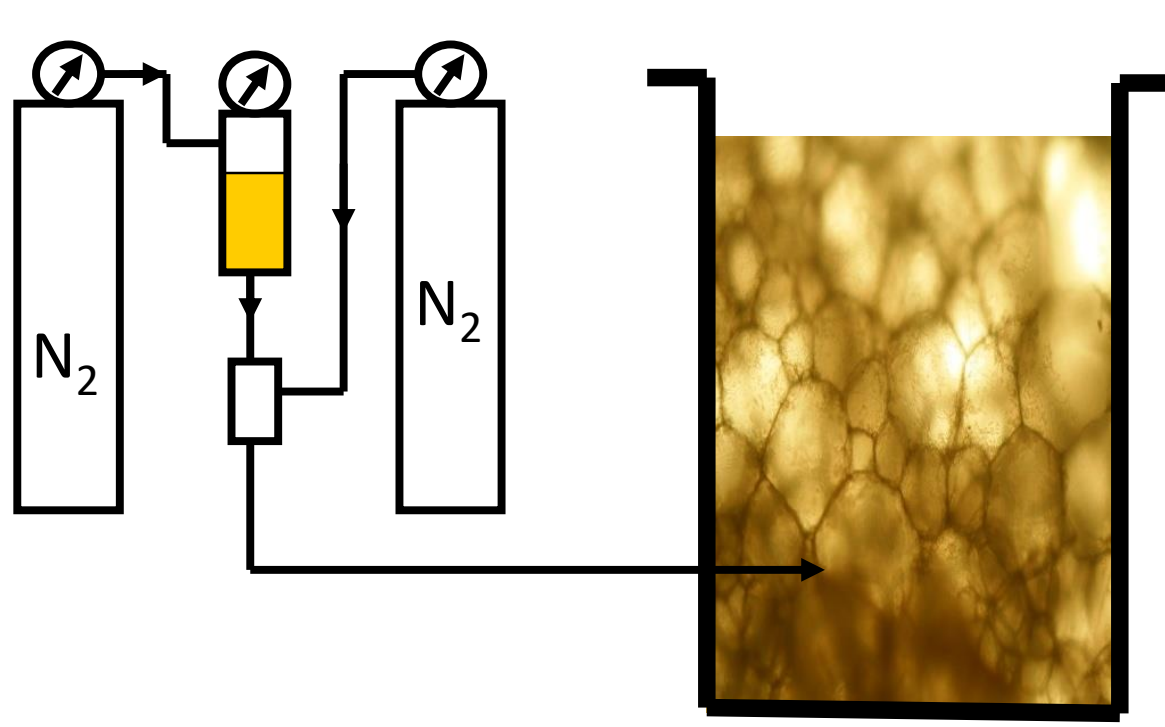

Foamability

$$
\begin{aligned}
& \text { c > } 0.3 \text { wt. \% - shaking } \\
& \text { c > } 0.1 \text { wt. } \% \text { - t. mixing }
\end{aligned}
$$


Foams: MLS multiple light scattering Diffusing Wave Spectroscopy

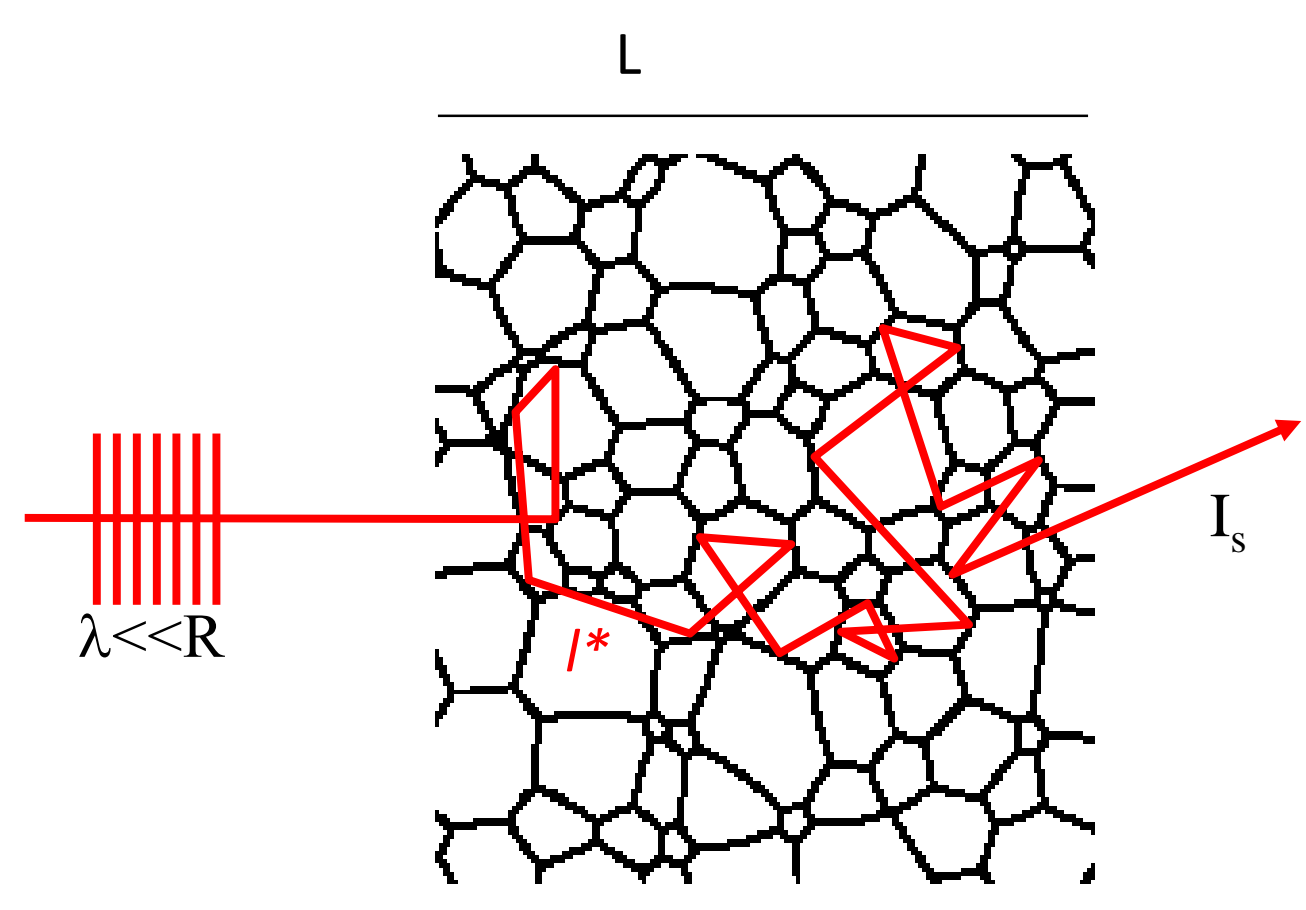

$\mathrm{I}_{\mathrm{s}} \sim / * / L \sim R / \sqrt{ } \varepsilon$

$I^{*}=$ photon transport mean free path

No absorption and optically thick medium

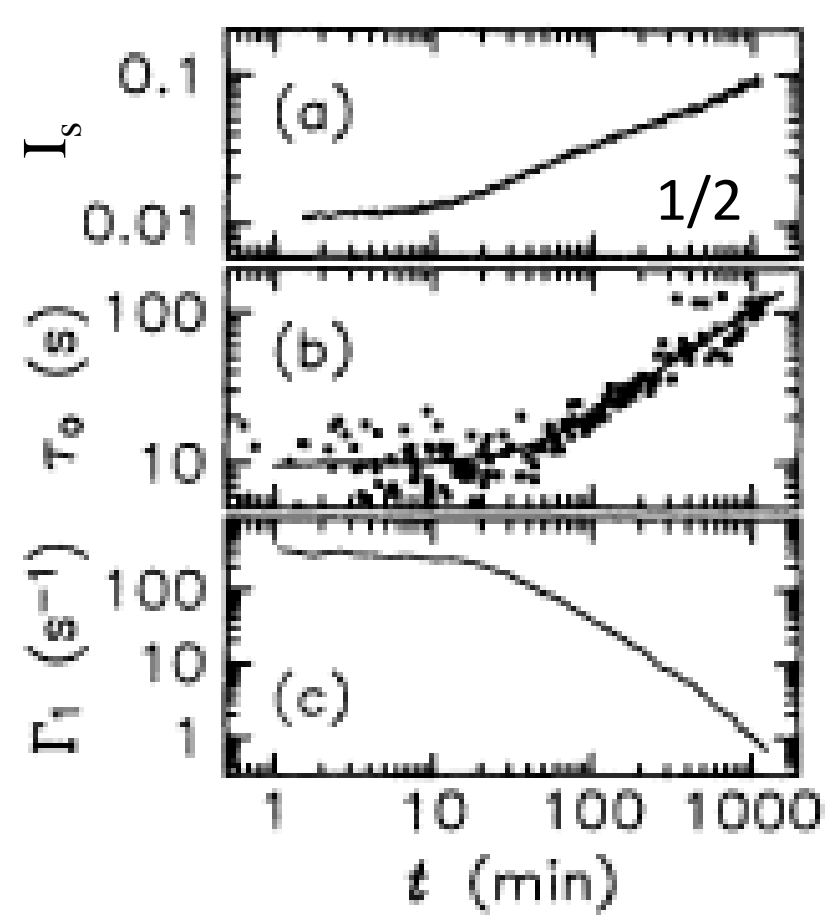

correlation function $\left\langle\mathrm{I}_{\mathrm{s}}(\tau) \mathrm{I}_{\mathrm{s}}(0)>\right.$ single $\tau_{0}$ relaxation time $\Gamma_{1}$ first cumulant 


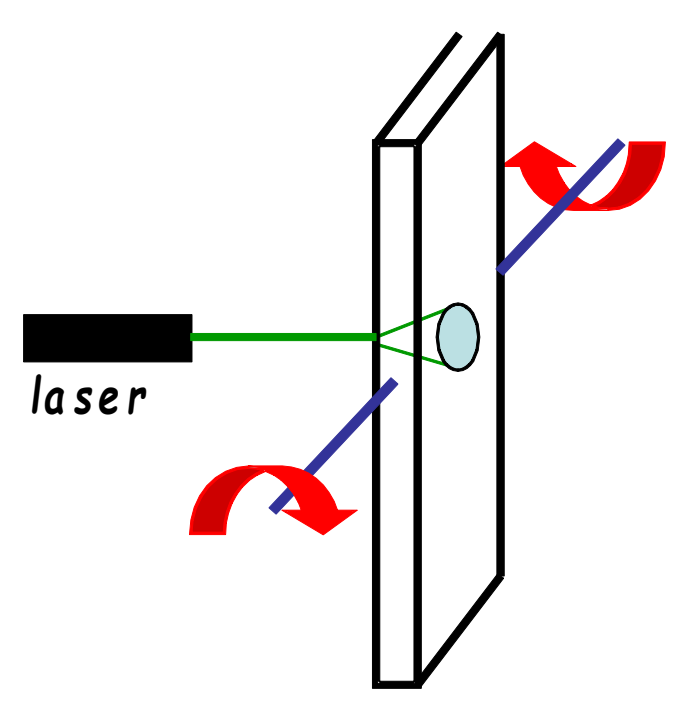

A.Saint Jalmes et al 2005.

A. Cervantes-Martinez et al. 2008
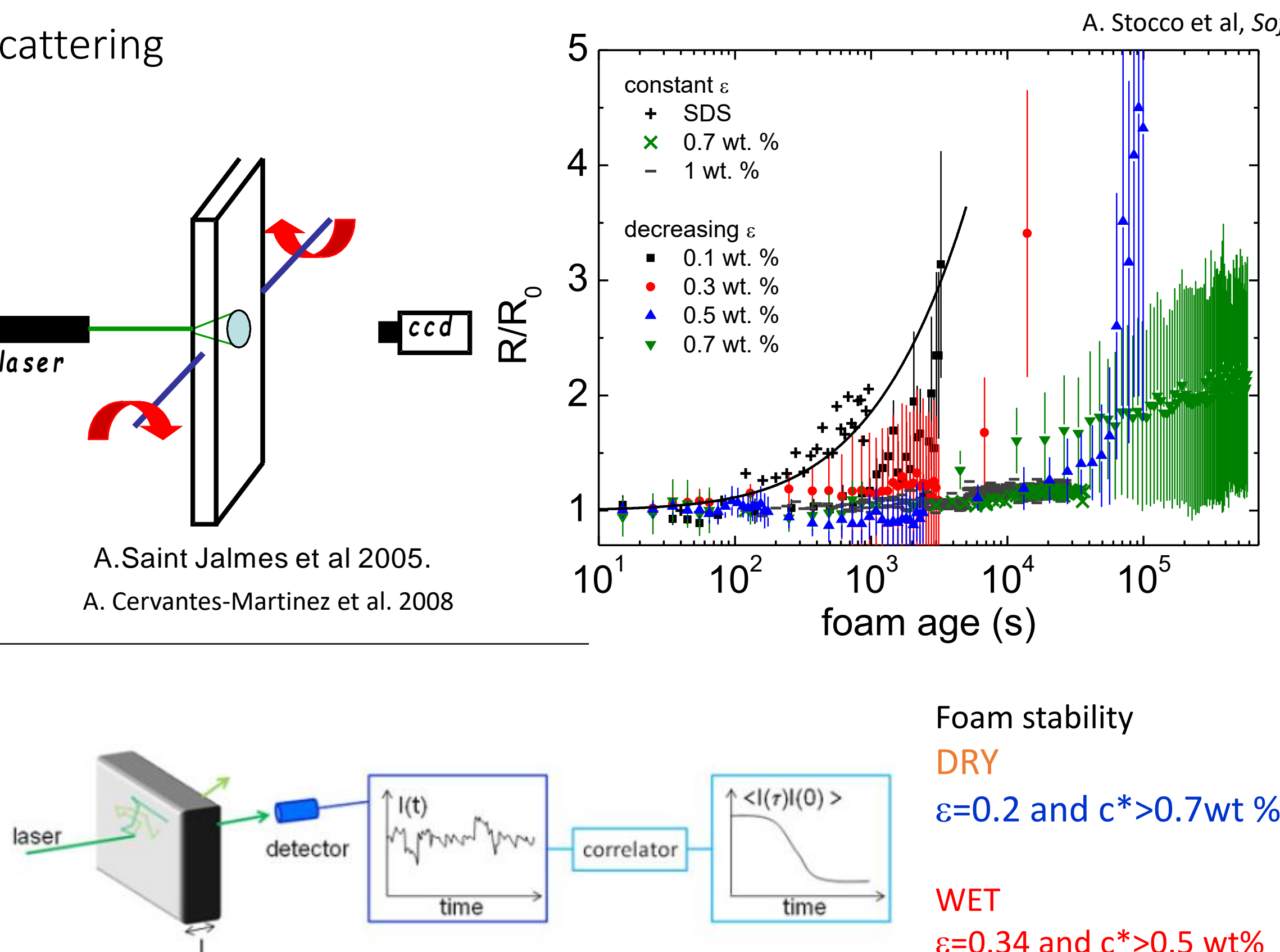

Foam stability

DRY

$\varepsilon=0.2$ and $c^{*}>0.7 w t \%$

WET

$\varepsilon=0.34$ and $c^{*}>0.5$ wt $\%$ 
Wet Foams: $\mathrm{X}-\mu \mathrm{CT}$

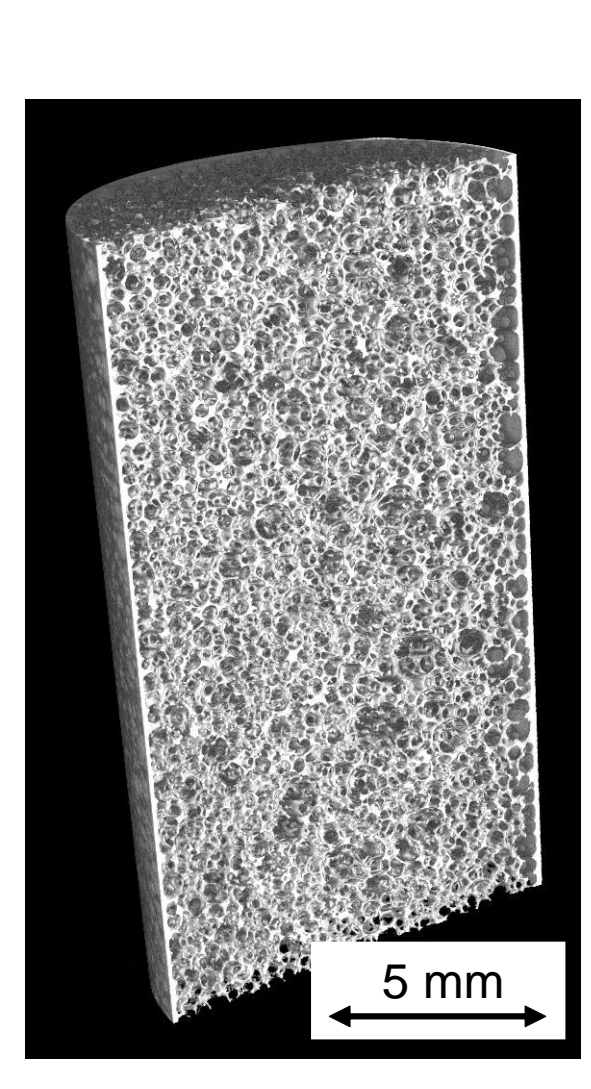

$$
\begin{aligned}
& \text { Age }=10^{4} \mathrm{~s} \\
& \varepsilon=0.25
\end{aligned}
$$

Lab $\mu \mathrm{CT}$

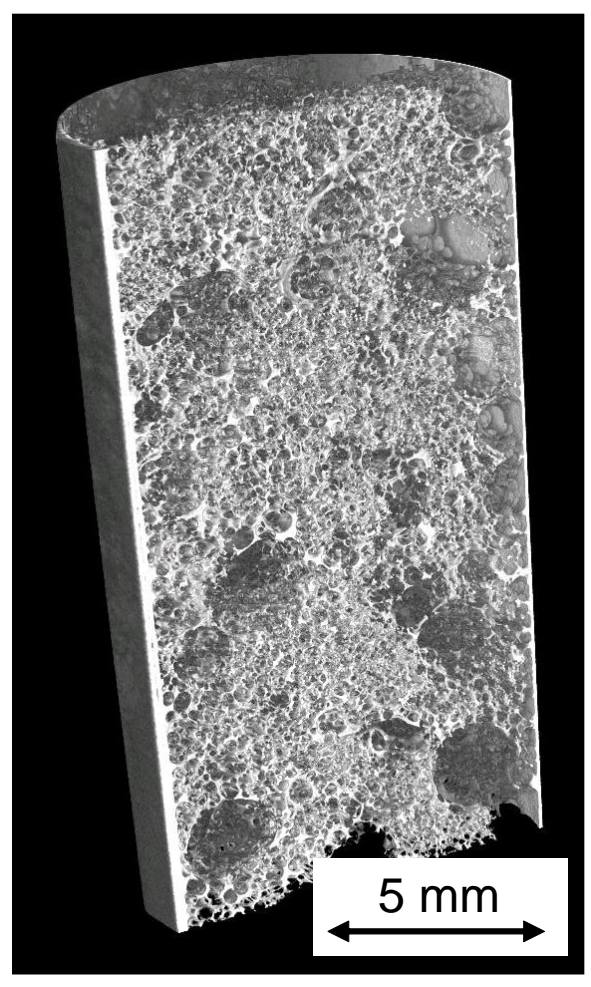

$$
\text { Age }=10^{5} \mathrm{~s}
$$$$
\rightarrow
$$

0.22
Synchrotron $\mu \mathrm{CT}$

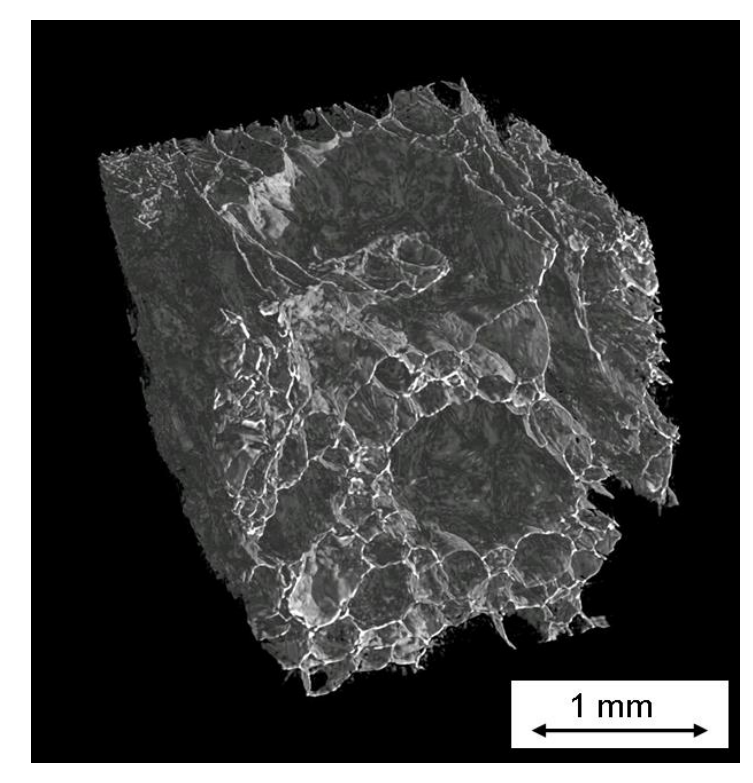

Age $=10^{6} \mathrm{~s}$ $\varepsilon \sim 0$

$c=0.7$ wt. $\%$

$c=0.9$ wt. $\%$ 
One population, mean diameter $=100 \mu \mathrm{m}$

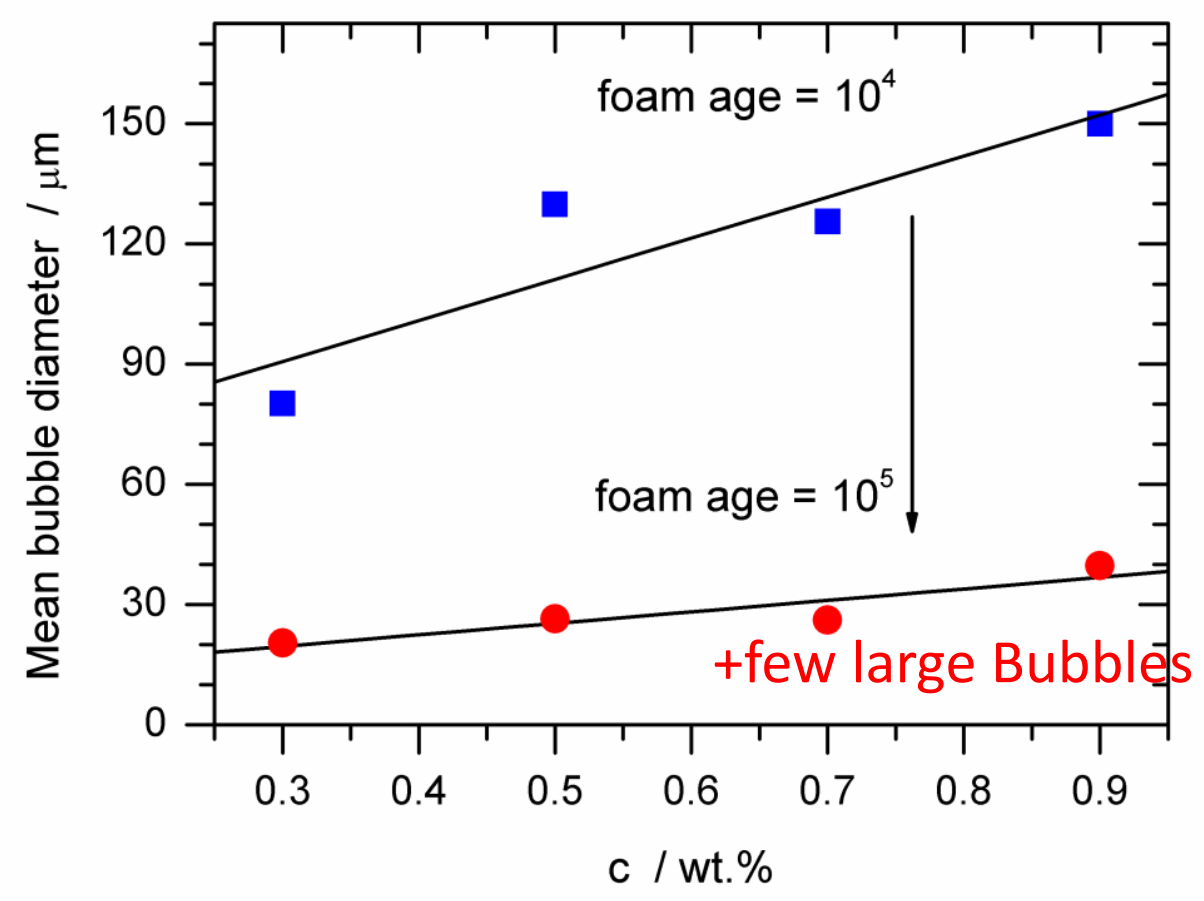

DRY Foams

Final mean diameter $=25 \mu \mathrm{m}$

Low surface coverage! $4-8 \mathrm{mg} / \mathrm{m}^{2}$

Loose network (a)

\section{WET Foams}

Appearance of few big bubbles Final mean diameter $=25-30 \mu \mathrm{m}$

\section{Arrested coarsening mechanism}

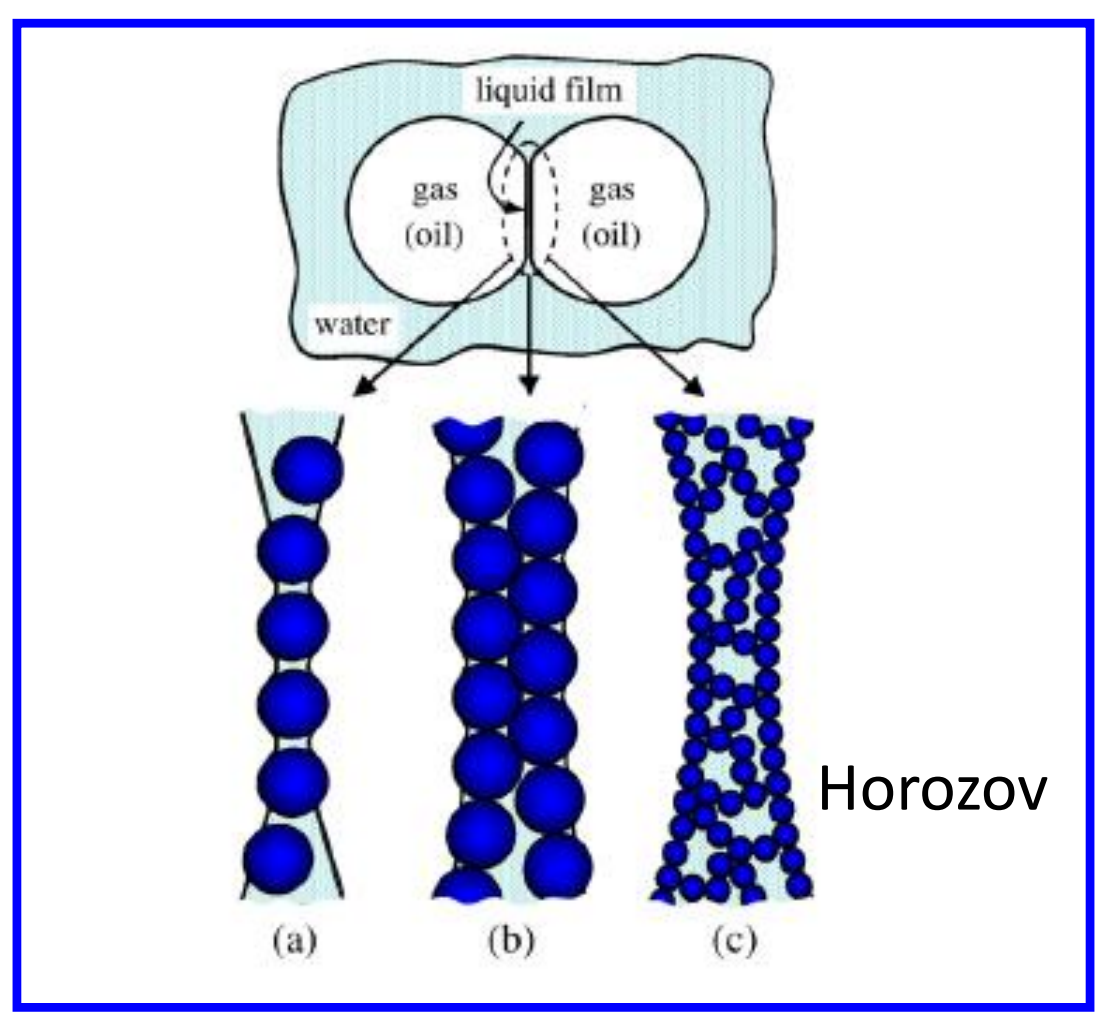

A. Stocco et al, Soft Matter 2011 


\section{Summary Part 3: Foam stability and dynamics}

- Hierarchical levels in foams (macroscopic, bubble, film, interface)

- For c>c*, particle stabilized foams are infinitely stable

- Surface elasticity and surface tension $\gamma$ and the formation of an interfacial network rule foam stability vs. Coarsening.

- Particle networks in films and at the interface are observed
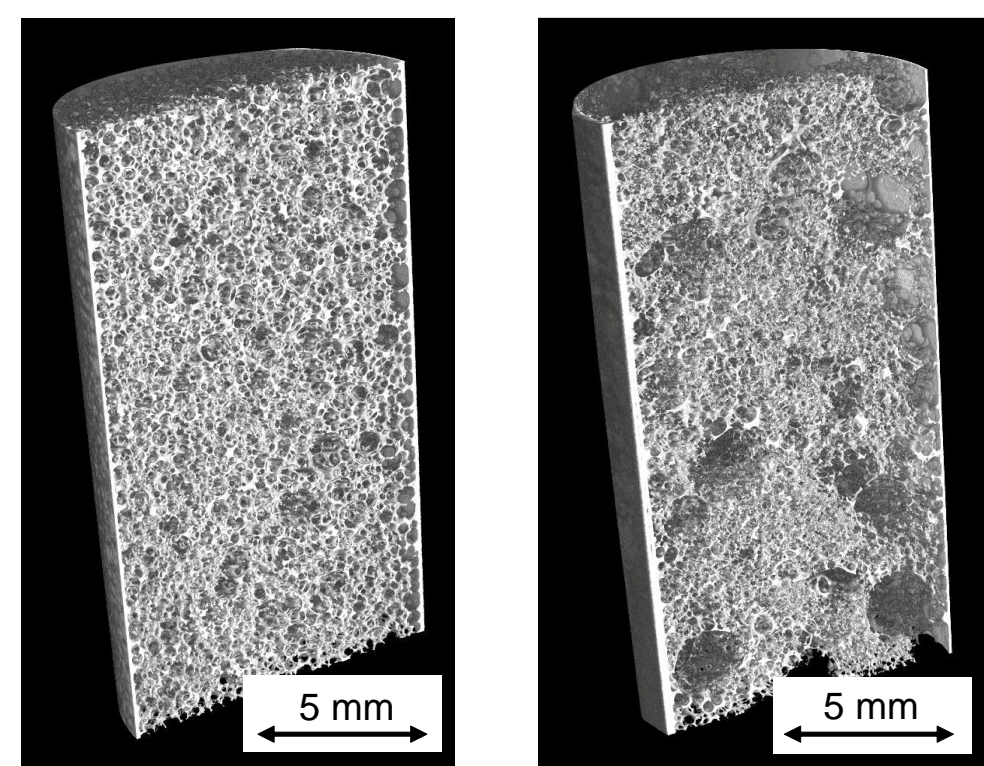
Thank you! 